Advances in analytical tools and current statistical methods used in ultra-high-performance liquid chromatography-mass spectrometry of glycero-, glycerophospho- and sphingolipids

\author{
Avela, Henri F.
}

2020-11

Avela , H F \& Siren , H 2020 , ' Advances in analytical tools and current statistical methods used in ultra-high-performance liquid chromatography-mass spectrometry of glycero-, glycerophospho- and sphingolipids ' , International Journal of Mass Spectrometry , vol. 457 , 116408 . https://doi.org/10.1016/j.ijms.2020.116408

http://hdl.handle.net/10138/333288

https://doi.org/10.1016/j.jms.2020.116408

cc_by_nc_nd

acceptedVersion

Downloaded from Helda, University of Helsinki institutional repository.

This is an electronic reprint of the original article.

This reprint may differ from the original in pagination and typographic detail.

Please cite the original version. 


\section{Manuscript Details}

Manuscript number

Title

Article type

Keywords

Taxonomy

Corresponding Author

Corresponding Author's Institution

Order of Authors

Suggested reviewers
IJMS_2020_121_R1

Advances in analytical tools and current statistical methods used in ultra-highperformance liquid chromatography-mass spectrometry of glycero-, glycerophospho- and sphingolipids

Review Article

Lipidomics; mass spectrometry; ultrahigh performance liquid chromatography; nano-liquid chromatography; chemometrics; statistical methods; multicomponent analysis

Liquid Chromatography Mass Spectrometry, Clinical Analysis, Analytical Chemistry Instrumentation, Bioanalysis, Chemometrics

Henri Avela

Helsinki University

Henri Avela, Heli Siren

Alexia Gloess, Alexandra Antunes, Ho Leung Ng, Jonathan Bones

\section{Submission Files Included in this PDF}

\section{File Name [File Type]}

Cover Letter.pdf [Cover Letter]

Cover letter_Revised.pdf [Response to Reviewers]

IJMS_Review_Revised_Changes.pdf [Revised Manuscript with Changes Marked]

IJMS_Highlights.pdf [Highlights]

First page_Revised.pdf [Abstract]

Graphical Abstract.tiff [Graphical Abstract]

IJMS_Review_Revised_NoChanges.pdf [Manuscript File]

Figure 1.tif [Figure]

Figure 2.tif [Figure]

Figure 3.tif [Figure]

Figure 4.tiff [Figure]

Tables_Figures_Revised.pdf [Table]

Declaration_of_competing_interests.pdf [Conflict of Interest]

To view all the submission files, including those not included in the PDF, click on the manuscript title on your EVISE Homepage, then click 'Download zip file'.

\section{Research Data Related to this Submission}

There are no linked research data sets for this submission. The following reason is given:

Data will be made available on request 
To International Journal of Mass Spectrometry (IJMS)

Helsinki, March 27th 2020

Dear Editor,

Enclosed, please, find an electronic submission of a review manuscript entitled

Advances in analytical tools and current statistical methods used in ultra-high-performance liquid chromatography-mass spectrometry of glycero-, glycerophospho- and sphingolipids written by Henri Avela and Heli Sirén for publication in International Journal of Mass Spectrometry.

We have broadly reviewed the status of current RPLC-MS analytics in the light of UHPLC-MS studies between the time frame of 2017-2019 to elucidate both standardized and variable aspects practiced by researchers for both identification and quantitation in lipidomics.

We focus to the importance of the topic and to orient current method validation towards factors (e.g. gradient, adducts) yet undecided in the lipidomic community and to clarify the choices made in lipidomic analyses. Moreover, complex lipidomic data demands high-throughput data processing as well as the appropriate chemometric and statistical tools, which are also represented in the review.

The manuscript contains 4 figures and 5 tables. Among them, Figure 4 needs permission for reprinting.

Looking forward to receive your decision about the review paper.

Sincerely,

Henri Avela and Heli Sirén 


\section{Cover letter}

The response letter to the reviewer's comments on the manuscript

IJMS_2020_121

Advances in analytical tools and current statistical methods used in ultra-high-performance liquid chromatography-mass spectrometry of glycero-, glycerophospho- and sphingolipids

by Avela and Sirén

To the Editor of International Journal of Mass Spectrometry

Professor Zheng Ouyang

Please, find below our responses to the comments of the reviewers about the first version of the manuscript IJMS_2020_121.

\section{Response to the reviewers' comments}

Comment 1: This review contains a very wide coverage of literature of LC-MS methods for lipids analysis, this makes it too lengthy to read. I suggest the authors to remove basic knowledges of lipid nomenclature, liquid chromatography and mass spectrometry.

Response 1: The exposition on basic knowledge concerning the topics lipid analysis, LC and MS were minimized. However, some paragraphs were kept to preserve the text's flow and readability.

Comment 2: Besides, discussion of challenges specific to the analysis of each type of biological sample should be added.

Response 2: A brief introduction to biofluids, tissue and cell culture sample preparation was added to the Chapter 'Instrumentation'. Biological samples and their sample preparation is discussed elsewhere, since the main text was asked to be minimized.

\section{Reviewer \#1:}

Comment 1: Although LC-MS are widely used for lipid analysis, shotgun lipidomics methods were also developed and employed into various biological samples.

Response 1: We have included our comments and compare shortly direct infusion techniques with HPLC-MS in the Chapter on 'Quantification of lipids'. We think that an extra paragraph on the mehod comparison will increase the basic information, which was suggested to be removed or decreased in the main paper 
Comment 2: I understand that emphasizing UPLC can make this review more focusing, however, since many MS methods are developed based on HPLC, some very important MS methods for lipid analysis may be omitted.

Response 2: We have acknowledged this limitation in the 'Discussion' section and addressed some techniques mentioned by reviews on lipidomic MS-analysis.

Comment 3: During past few years, more attentions were paid onto lipid isomer analysis by developing new MS methods, e.g. $\mathrm{C}=\mathrm{C}$ isomer and sn-isomer analysis. Related references should be included.

Response 3: The topic was extended upon up-to-date methods (e.g. Paterno-Büchi acetone derivatization) in the end of the Chapter 'Identification of lipids'. Furthermore, some methods were already mentioned in the text (e.g. SWATH, SONAR, PRM in the 'Introduction' and SWARM in the 'Instrumentation' chapter)

Comment 4: Reference [15] is a review paper, it cannot be used a source of experimental conditions in Table S1. Acetone has been reported as a major component of the mobile phase both for HILIC (2019) and RPLC (Anal. Chem. 2020) separation phospholipids.

Response 4: The study on buttermilk by Castro-Gómez et al. [15] includes an experimental section and results discussing them. Anyhow, acetone is now mentioned and cited as a organic phase in the limitations-paragraph of the Discussion-section (side note: UHPLC articles barely noted this solvent, though it must be used for the Paternó-Büchi photochemical derivatization)

Comment 5: Quantitation of lipids in biological samples should be discussed.

Response 5: A chapter ('Quantitation of lipids') was included to address the topic and its challenges. Furthermore, additional information had to be added for the normalization (i.e. standard usage) of lipidome data. 


\title{
Advances in analytical tools and current statistical methods used in ultra-high- performance liquid chromatography-mass spectrometry of glycero-, glycerophospho- and sphingolipids
}

Henri F. Avela and Heli Sirén

Department of Chemistry, University of Helsinki, P.O. Box 55, FI-00014 University of Helsinki (FI-00560 Helsinki), Finland

Correspondence: Henri F. Avela (MSc), Faculty of Science, Department of Chemistry, University of Helsinki, A.I. Virtasen Aukio 1 (P.O. Box 55), FI-00014 University of Helsinki, Finland; Heli Sirén (Assoc. Professor, Doc, PhD), Faculty of Science, Department of Chemistry, University of Helsinki, A.I. Virtasen Aukio 1 (P.O. Box 55), FI-00014 University of Helsinki, Finland, heli.m.siren@helsinki.fi

\begin{abstract}
The review concentrates on the properties of analytical and statistical ultrahigh-performance liquid chromatographic (UHPLC) - mass spectrometric (MS) methods suitable for glycero-, glycerophospho- and sphingolipids in lipidomics published between the years 2017-2019. Trends and fluctuations of conventional and nano-UHPLC methods with MS and tandem MS detection were observed in context of analysis conditions and tools used for data-analysis.

Whereas general workflow characteristics are agreed upon, more details related to the chromatographic methodology (i.e. stationary and mobile phase conditions) need evidently agreements. Lipid quantitation relies upon isotope-labelled standards in targeted analyses and fully standardless algorithm-based untargeted analyses. Furthermore, a wide spectrum of setups have shown potential for the elucidation of complex and large datasets by minimizing the risks of systematic misinterpretation like false positives. This kind of evaluation was shown to have increased importance and usage for cross-validation and data-analysis.
\end{abstract}

\section{Keywords}

Lipidomics, mass spectrometry, ultrahigh performance liquid chromatography, nano-liquid chromatography, chemometrics, statistical methods, multicomponent analysis 


\section{Highlights}

- Method development and application enhancements in lipidomics

- The review sums up chemometric and statistical methods for current lipidomics

- State of the art data collection and evaluation is discussed

- Identification/quantitation of biological lipids

- Tandem MS data-independent and data-dependent analysis

\section{Abbreviations}

ACP acyl carrier protein, AF4 asymmetric flow field flow fractionation, ANOVA analysis of variance, AUC area under curve, CARS coherent anti-Stokes Raman scattering, CCS collision cross-section, CN total carbon, CRS coherent Raman scattering, DB double bond, DDA data dependent acquisition, DESI desorption electrospray ionization, DG diacylglycerol, DIA data independent acquisition, DIMS direct infusion MS, FDR false discovery rate, FA fatty acyl, FFA free fatty acid, GC gas chromatography, GL glycerolipid, GP glycerophospholipid, HCA hierarchical cluster analysis, i.d. internal diameter, IMS ion mobility spectrometry, HPLC liquid chromatography, LESA liquid extraction surface analysis, m/z mass-to-charge [ratio], LSI Lipidomic Standards Initiative, HR/LRMS high/low resolution mass spectrometry, MS ${ }^{\mathrm{E}}$ all ions scans, MS/MS tandem mass spectrometry, MS/MS/MS second-generation fragmentation mass spectrometry $\mathrm{MS}^{\mathrm{n}}$ on-line coupled mass spectrometry system, MSI mass spectrometric imaging, m/z mass-to-charge ratio, NP normal phase, NSI nano-electrospray ionization, nESI negative ion mode in electrospray ionization, OPLS-DA orthogonal projections to latent structures discriminant analysis, (L)PA (lyso-)phosphatidic acid, (L)PC (lyso-)phosphatidylcholine, (L)PE lyso-phosphatidylethanolamine, pESI positive ion mode in electrospray ionization, (L)PG (lyso-) phospatidylglycerol, (L)PI (lyso-)phosphatidylinositol, NP-HPLC normal phase liquid chromatography, PIS precursor ion scan, PLS-DA partial least squares discriminant analysis, PRM parallel reaction monitoring, (L)PS (lyso- 
)phosphatidylserine, QqQ triple quadrupole mass analyzer, QTOF quadrupole - time of flight, ROC receiver operating characteristics curve, RP-HPLC reversed phase liquid chromatography, RT retention time, SP sphingolipid, SFC supercritical fluid chromatography, SWATH sequential window acquisition of all theoretical fragment ion mass spectra, SWARM sliding window adduct removal method, TG triacylglycerol, TOF time of flight mass analyzer, UHPLC ultra-high performance liquid chromatography, VIP variable importance projection

\section{Introduction}

Recent lipid research has emerged due to improved multidimensional computer algorithms and highly efficient commercial, open-source and in-house software platforms. Furthermore, data libraries for automated routine searching of mass spectra is adopted for lipid identification. International organizations have started together to harmonize knowledge about lipidomics. Especially, the Lipidomics Standard Initiative (LSI, https://lipidomics-standardsinitiative.org/) found under the International Lipidomic Society (https://lipidomicssociety.org/about/) and Lipid Home (https://www.lipidhome.co.uk/) strive to standardize lipidomic information with the globally acknowledged platform Lipid MAPS (https://www.lipidmaps.org/). Furthermore, Sumner et al. [1] have stated minimal requirements for retraceable lipid analyses, [1] which are important for integrated research in lipidomics. This review on lipid investigatios encompasses research conducted with ultra-high performance mass spectrometry (UHPLC-MS) during 2017-2019 [2-79]. To clarify, this review uses the term high performance liquid chromatography (HPLC) to describe both conventional HPLC and UHPLC. However, blindspots of exclusive UHPLC-research are attempted to be covered in the Discussion-section.

Particularly, half of the reviewed studies deal with computerized platforms to identify lipid species of several classes. These computerized platforms have successfully enabled data handling with in silico analyses, commonly generalized as machine learning algorithms. After 
all, the most popular means of comprehensive identification in -omics research is on-line coupled mass spectrometry systems (MS ${ }^{\mathrm{n}}$, e.g. tandem mass spectrometry [MS/MS]), which demand extensive and thorough data-handling. In the field of lipidomics, $\mathrm{MS}^{\mathrm{n}}$ is often divided into data-dependent acquisition (DDA) and data-independent acquisition (DIA). Here, DDA is typically defined as fragmentation of only targeted precursors, as is the case in parallel reaction monitoring (PRM) [15], [44], [45]. It is a technique, in which all fragments of the chosen lipid precursors are measured. In DIA, all available lipid precursors are fragmented and measured in a full scan with a set of collision energy (CE) or energies, detecting a substantial amount of lipid adducts fragmentation patterns, e.g. in an all ions scan $\left(\mathrm{MS}^{\mathrm{E}}\right)$ [9-15], [32-34], [55-61], [68], [74].

Recent DIA research were applied in a novel MS technique called sequential window acquisition of all theoretical fragment ions spectra (SWATH MS) [6], [44], [73-76] and similarly, with QTOF-specific SONAR technology [69]. The consecutive fragmentation or scan of precursors (i.e. PRM for DDA and SWATH or SONAR for DIA) and their product ions provide cleaner MS spectra in favour of library searches, an improved detection rate, broadened range, and expanded specificity in any given fragmentation frame compared to other methods [34], [44], [73], [69]. Different MS and MS/MS techniques are compiled in Table 1. When significant, instrumental and experimental conditions for glycero-, glycerophospho- and sphingolipids are focused on and referred to [80-88].

\section{Fatty acids, lipids and metabolites of the survey}

Fatty acids are synthetized in cells and their cell membranes, endoplasmatic reticulum, Golgi apparatus, and mitochondria [89], [90]. Most lipids are products of free fatty acids in presence of coenzyme A and NADPH [91]. This literature survey deals with a short area of lipidomics and contains commonly studied lipids, e.g. glycerolipids (GLs) like mono- (MG), di- (diacyl-, DG), and triacylsubstituted (triacyl-, TG) glycerols. [91] To look the structural challenges of 
lipids in analytics, Figure 1 illustrates the functionalities of TG lipids. Glycerophospholipids (GPs) from GLs are also included, since they are frequently detected like the most abundant lipids in eukaryotic cells, i.e. phosphatidylcholine (PC) and phosphatidylethanolamine (PE) [92]. Other GPs dealt in this review include phosphatidic acid (PA), phosphatidylserine (PS), phosphatidylinositol (PI), phosphatidylglycerols (PGs), and PG-derived cardiolipins (CLs). [92] To observe the challenges of separation and identification issues some glycerolipids and glycerophospholipids are illustrated in Figure 2.

GPs may appear with fatty acids which both are bound with ester or ether groups, the latter being defined as plasmalogens (PLs) [92]. PLs are detected and identified either by an alkenyl linker with an oxygen (e.g. PI[O-18:0/17:0]) or with a phosphorous (e.g. PI[P-18:0/17:0]) [92]. Those structural hetero atoms help mass spectrometric detection, although there may not be improvements in chromatographic separation.

An interesting group of surveyed lipids is sphingolipids (SPs, Figure 3, [28]). They have a basic backbone with various kinds of lipids. The backbone is formed from serine amino acid and a long-chain fatty acyl catalyzed by coenzyme A in mammals [92]. The analytics is focused to ceramides (Cer), which have amine-bonds with fatty acids. To sum up, interest is focused on esterified fatty acids with glycerol head groups (glycerolipids, glycerophospholipids) or a sphingosine headgroup (sphingolipids), but not a sugar backbone (saccharolipids, a topic of worth its own review). Other groups left out are defined by characteristic hydrocarbon structures, i.e. fatty acyls, prenol lipids, sterols and polyketides [94]. More analysis on lipid classes and metabolism is discussed elsewhere [101]

\section{Instrumentation}

In lipidomics, a wide range of articles introduce new or improved methods which are validated with UHPLC-MS, capillary-UHPLC-MS (nUHPLC-MS or nano-UHPLC-MS) instruments (Table 2). Most of these studies concentrate on sample preparation [14], [20], [46], [66], 
development of chromatographic methods [9], [22], [27], [45], MS/MS identification [7], [16], [23], [27], computational methods for improvement of measurement performance [17], and data analysis [21], [25], [96]. Recent challenges in HPLC-MS seem to be fluctuation (repeatability) of analysis [97], [98] results, which hinder reliable identification and interlaboratory tests of lipids. Since in chromatographic environments elution of different lipid types and their species may differ [98], deconvolution of data via manual or computational analysis may be the only reasonable compromise.

The reviewed papers show that all lipid analyses have fluctuation of lipid composition and intensity based on the fingerprint profiles of different biological matrices. These observations can be explained by distinctive matrix effects, which in turn inform that there is a specific need of sample preparation before analyses. Usually, lipidome studies are done with simplified sample preparation to avoid recovery losses in clean-up steps. [99] Mostly, the clean-up steps are protein precipitation and extraction of solid matrix compounds (e.g. biological tissues) and fluids (e.g. plasma, serum, lavages, cell suspensions and supernatants).[99] Furthermore, solid materials may trap internal standards and analytes, which increases variation of results in quantification and leads to less accuracy and precision of the methods. [100] Sample preparation of biological and clinical samples is discussed more elsewhere [101].

Though reconstitution with the most used polar acetonitrile - water mixture $(60: 40, \mathrm{v} / \mathrm{v})$ and organic isopropanol - acetonitrile $(90: 10, \mathrm{v} / \mathrm{v})$ eluents is practical, none of these dissolve lipids comprehensively. For instance, acetonitrile - water at 60:40 (v/v) recovers STs and TGs incompletely. [45] Furthermore, Danne-Rasche et al. [45] observed a distortion or even loss of lipids with i-propanol - acetonitrile mixture $(9: 1, v / v)$, when the eluent was used in nanoUHPLC. Thus, lipids need to be reconstituted into a standardized mixture such as butanol - ipropanol - water $(8: 23: 69, \mathrm{v} / \mathrm{v} / \mathrm{v})$ [45] prior to injection. The solvent composition is important for lipid solubility. Due to high solvent volumes from pretreatment processes at the end of 
sample preparation, all lipid samples need to be dried for getting concentrates of HPLC-MS research. Furthermore, concentrates are needed to measure multidimensional MS spectra to obtain accurate identification and algorithm-based data handling of features.

To reduce systematic noise and other disadvantages (e.g. adsorption, peak broadening, adduct formation) in chromatographic data has been attempted via computational methods, i.e. with the sliding window adduct removal method (SWARM). [102] SWARM is based on the systematic interference caused by multiple adduct ions of the same analyte. The interference induces systematic noise patterns that may be excluded to enhance accurate mass acquisition for metabolites. Then, the adduct signal overlap correction for low-to-moderate resolution mass spectra could be utilized.

Modern instrumental separation and detection methodologies are still on-going needed to avoid frequent appearance of false negative lipid identifications and to establish sufficiently reliable and label-free (i.e. standard-free) methods. Identification of lipids showed to be improved by multiply usability of analytical variables and by using more independent properties, such as chromatographic retention times (RT) and mass spectrometric mass-to-charge ratios $(\mathrm{m} / \mathrm{z})$ of lipid ions/adducts, fragmentation/isotope patterns, and collision reactions. Reliability for targeted lipid species identified by internal standards and calibrants have made lipid analyses possible in quite many case, but especially non-targeted lipid analyses demand the super power and speed of computers with algorithm-based libraries.[96], [103], [104] Thus, the analyses can be independently conducted without potent analytes. Evidently, the computerized methods have utilized automated systems when internal standards for normalization are neglected. [2], [3], [10], [11], [13], [14], [16], [17], [20], [21], [22], [24], [26], [30], [32-36], [38], [44], [4 6], [47], [59], [63], [66], [73].

\subsection{UHPLC}


HPLC techniques are preferred for their ability to enhance identification by predictable retention time patterns [98], [105] and reduced mass ambiguity [106]. In lipid research, the sub-method UHPLC is preferred over HPLC due to its lower dispersion, substantial decrease in solvent usage, shorter analysis times without compromising resolution, higher resolution performance due to smaller (sub-2 $\mu \mathrm{m}$ ) fully porous particles or (sub-3 $\mu \mathrm{m}$ ) core-shell material [73] in columns (enabled by higher pressure capabilities) and enhanced retention precision. [107]

Our dataset [2-79], [108] informs current lipidomic UHPLC-MS separations to be primarily done with additive-assisted reversed phase liquid chromatography (RP-HPLC) hyphenated with separate experiments on positive electrospray ionization (pESI) and negative electrospray ionization (nESI). Though ESI was predominantly used for UHPLC-MS, some atmospheric pressure chemical ionization (APCI) studies were also conducted. [23], [44], [47]. ESI as a 'soft' and APCI as a 'hard' ionization technique, respectively, are suitable for supporting lipid identification. [23] In respect of that, Beccaria et al. [23] developed a method suitable for both detection approaches with no need to change HPLC parameters.

Elution of lipids in HPLC is done in many elution models (e.g. isocratic elution, curved gradient following an exponential or logarithmic function, stepped gradient, linear gradient, etc.). The suitable stationary and mobile phases with methods are also found in Table 1. More detailed information on stationary and mobile phases as well as lipid adduct concentrations is in Supplementary (Table S1).

\subsubsection{Reversed phase separation in HPLC}

In lipidomics the commonly used RP-HPLC separation methods are based on the interaction of a nonpolar stationary phase with nonpolar lipid analytes. Nonlinear or second-degree curves between the retention times and total carbon $(\mathrm{CN})$ double bonds $(\mathrm{DB})$ are used for quantitative calculations. [9], [16], [98], [105] For example, they help to differentiate possible sodium 
adducts from similar exact masses (e.g. $[\mathrm{PC}(38: 4)+\mathrm{Na}]^{+}$and $\left.[\mathrm{PC}(40: 7)+\mathrm{H}]^{+}[9]\right)$. However, retention time prediction can be complicated due to $\mathrm{pH}$ dependent speciation [109], experimentally made validation and multi-step liquid gradients [23]. Separation of polar components (i.e. early retaining lipids) can be affected by the mobile phase gradient. When elution is started with high percentage of organic mixture, polar compounds are longer adsorbed and retained on RP-HPLC phases. [110] However, changes of eluent polarity are interlinked with the ionization rate of polar lipids, which in turn can have an impact on elution. [110] RP-HPLC with polar solvents (i.e. non-aqueous RP-HPLC) is enabled by polymer-based columns which are end-capped by a methyl or hydrogen group. [110] Some column materials are based on modified silica, which when not shielded are short-periodically used with water modified organic solvents due to free silanol groups having the attractive functions. [110] In contrast to phenomena in reversed phases, normal phases in HPLC (NP-HPLC) typically separates analytes containing polar functionalities having silanol, amino- or diol groups. [111] NP-HPLC is excluded from the review, since only a single article on NP-HPLC with amide column was observed during 2017-2019 on lipid separation [31].

Since even the variation among lipid class species can be substantial, it is not surprising that the polarity of their classes varies a lot. Tumanov and Kamphorst [85] (Figure 4) demonstrated the lipid-subclass range of four distinct chromatographic approaches, which are divided to two groups (one a RP, one a NP separation strategy in each group). The subclasses in (A) include lipids of the nonpolar kind, whereas the separations of $(\mathbf{B})$ are modelled to the polar lipid subclasses. Particularly, polar (lyso-forms, MGs, sphingosine-related compounds, FAs, acyl carnitines and acetyl coenzyme A) and mid-polar (PS, PG, PI, Cer) lipids seem to be species often analysed with the negative ionization mode, though more species are primarily found with positive ionization. From the GLs only MGs have FA chains with 16 counts, i.e. they are small enough to be rather polar. As a thumb rule, SPs are on the mid-polar lipid spectrum, when 
they have amide-bound FA chains. [85] Thus for reliable research data, accurate UHPLC-MS methods are needed to identify the individual lipid class species.

\subsubsection{HILIC}

Essentially, hydrophilic interaction liquid chromatography (HILIC) columns are micro-bore columns with stationary phases of mixed hydrophilic interactions for nonpolar compounds and hydrophobic interactions for polar compounds. However, nonpolar lipids (e.g. CEs, TGs) and lipids with only one hydroxyl group (e.g. Cer, DG, MG, and cholesterol) are often barely retained. [16] Nevertheless, HILIC is a well-established subclass of NP chromatography that allows usage of water as the eluent $(5-40 \%,>2 \%$ needed) as in contrast to conventional NP. [112] HILIC platforms are ideal for quantitation, since lipid class species co-elute with their respective calibrants. [109]

PAs and PSs species are known to have broad or barely detectable peaks in RP-HPLC, thus, HILIC is used. [112] Furthermore, as PAs and LPAs co-elute among major lipid components (e.g. PCs, PEs, SMs) in RP-HPLC. Because of that the comparatively lower abundance of lipids in this class, PAs and LPAs have no selective fragment to differentiate from other lipid classes. Thus, proper methods for PA/LPA separation have been specifically approached with HILIC.

\section{[113], [109]}

Though being a well-established technique for HPLC-MS quantitation, not many UHPLC analyses have used HILIC-columns for separation. [16], [47] Thus, HILIC applications are also excluded from the scope of this review.

\subsubsection{Nano-UHPLC}

In nano-HPLC [112] columns of sub-millimetre internal diameter (i.d.) are filled with conventional column packing materials. Published papers [41], [71], [24], [45], [42] inform only about using a loaded capillary (i.d.: 1-0.001 mm [114]) or a nanobore column (i.d.: 0.1$0.025 \mathrm{~mm}$ [114]). For comparison, a large majority of the lipid studies are done with the 
smallest possible narrow bore columns (2.1-4 mm). Surprisingly, along with nano-HPLC a low-resolution triple-quadrupole mass spectrometer was unequivocally preferred.

In contrast to a faster UHPLC separation, in nano-HPLC the "loading time" followed by the sample elution was extended due to the low flow rate restrictions $(0.3-0.35 \mu \mathrm{l} / \mathrm{min})$. [71]

Nano-HPLC methods show a great capacity for lipid identification with a low-resolution mass analyser, since then both an extremely broad identification range and high sensitivity at the low fmol-scale are gained using a low throughput (analysis time: 40-60 min). [41], [45] The result was that the low fmol range for almost every GLs, GPs, SPs and their lipid derivatives was reached in the positive ionization electrospray mode (pESI). For example, the calibration curve for a specific PE (17:0/14:1) demonstrated a linear relationship between 16-10000 fmol by pESI-MS detection and 0.64-2000 fmol by nESI-MS detection [41] By contrast, with HPLCESI-MS the linear dynamic range for the same analyte reached around 80-10000 fmol by pESIMS and 16-2000 fmol by nESI-MS. [45] Similarly, Kim et al. [115] achieved a LOD-range from 59 fmol (LPC(17:0)) to 507 fmol (LPG(14:0)) with untargeted nano-UHPLC-ESIMS/MS of lipoprotein by pESI and nESI ionization, respectively. [115]

\subsubsection{Mobile phases in hyphenation of HPLC with MS}

Mobile phases in HPLC are usually modified to help in lipid detection and their separation with HPLC. [112] As the sensitivity issues are concerned, composition of eluents is important to obtain stabile adduct ions in MS. [23] Volatile buffers (e.g. formic acid, acetic acid, or their ammonium salts [116]) are used in lipidomics methods. [113] However, earlier studies report lipid results with $5 \mu \mathrm{M}$ phosphate buffer (nanoelectrospray ionization) [9] and $5 \mathrm{mM}$ phosphoric acid [45]

In some cases, additives in HPLC eluents may cause analytes to become undetectable due to signal suppression. Cajka and Fiehn [117] studied the effects of five different modifiers in both pESI and nESI mode with two different HPLC columns filled with ethylene bridged hybrid 
(BEH) particles and the slightly better charged surface hybrid (CSH) particles. [117] Whereas the experiments could be concluded by choosing the optimal mobile phase modifier to be formic acid or formic acid/ammonium acetate for $\mathrm{pESI}$ and ammonium acetate for $\mathrm{nESI}$. Using a CSH C18 column with HPLC-pESI-MS studies Monnin et al. [118] reported about a further enhanced ionization efficiency by choosing $0.02 \%$ acetic acid as the additive in eluent. Whereas the signal for LPLs (except LSM) and GPs certainly increased by manifold, Cer and PCs experienced a decrease in peak area when compared with analyses data with $10 \mathrm{mM}$ ammonium acetate. [118] However, specific to PCs their carbonate ion adducts showed 20fold stronger signals compared to their ammonium adducts with CID-MS/MS. [119]

\subsection{Importance of mass spectrometry detection in lipid research}

Formation of adducts is dependent on the molecular structure and functional groups of a lipid (e.g. deprotonation of carboxyl groups in fatty acid with nESI). Lists of exact masses in MS and MS/MS, non-protonated adducts, possible collision energies and lipid classes/topics are listed in Table 3. Furthermore, sources like Lipid maps providing a free MS/MS prediction tool (http://lipidmaps.org/resources/tools/index.php) and a structure database library (http://lipidmaps.org/data/classification/LM_classification_exp.php) allow data handling in lipidomics.

MS advancements, such as a quadrupole Orbitrap mass analyzer (Q-Orbitrap) [9], [17], [20], [22], [27], [31], [46], [49], [51], [55], [56], [62-65], [77], [79], and NSI [24], [45], [71] have extremely increased resolution capabilities (theoretical plate number in HPLC >35 $000 / \mathrm{m}$ ) for lipid identification. However, compromises need to be done before the analyses, since either mass accuracy or ion resolution is emphasized depending on whether identification or quantitation is preferred. Furthermore, identification is improved with the help of orthogonal measurements, such as collisional cross-section (CCS) values with ion mobility spectrometry (IMS), achieved with a drift tube coupled with MS instruments, such as a quadrupole time of 
flight (QTOF) [6], [16], [25], [68]. Furthermore, the inclusion of trapped IMS (TIMS) combined with parallel accumulation serial fragmentation (PASEF) has emerged to enhance complementary MS/MS and IMS data. [126]

For high-resolution MS, an Orbitrap [2], [5], [34], QTOF [4], [6], [10-16], [21], [25], [28], [30], [32], [33], [36-40], [43], [44], [53], [54], [59-61], [66], [68-71], [71], AA073, [74], [75], AA076, [78] and QOrbitrap [3], [5], [9], [17-20], [22], [26], [27], [31], [34], [35], [46], [47], [49-51], [55-58], [60], [62-65], [72], [77], [79] were used. However, some studies settled for low-resolution MS, mostly with a triple quadrupole instrument [7], [8], [23], [24], [30], [4143]. Most rarely, pseudotargeted methods [21], [30], [54] (i.e. lipid identification with an in silico library), polarity switching [5], [17], [23], [41], [49], standalone nESI mode in MS [29], [43], [68], NSI [45], [71] and atmospheric pressure chemical ionization (APCI) [23], [44], [47] were used.

\subsection{Data acquisition and processing}

Identification and determination of lipids and their metabolites need commercial, open-source, and sophisticated tailor-made tools [127]. The statistical tools, analysis software, and algorithms enable visualization and perceiving of patterns from large datasets and raw data [103]. Metabolic profiling of MS data is done with multi-variant programs, such as Metaboanalyst, [10], [11], [17], [27], [32], [34], [37], [40], [46], [59], [63], [77] or MeV [3], [30], [65], [61], [79], [103],. All in all, reported software environments for data processing constitute mostly of R and SPSS languages.

Manual programming from the ground up or with borrowed code demands more computational expertise. Koelmel et al. [96] compared identification algorithms with the commercial R-based LipidMatch-tool, which is tailored for lipid identification softwares. [96] For a deeper understanding of differences in program functionalities, the article on "LipidMatch: an 
automated workflow for rule-based lipid identification using untargeted high-resolution tandem mass spectrometry data“" gives excellent information [96].

\section{Analysis}

\subsection{Identification of lipids}

Lipid identification can be enhanced by using information about the individual lipid itself, e.g. by increasing orthogonal information via "polarity switching" [5], [17], [23], [41], [49] instead of only using one ionization mode in the measurements or by the introduction of supporting measurements or (lipid class) expanding methods. Identification demands are partly already mentioned in Introduction and chapters, where chromatographic parameters are discussed referenced to resolution and sensitivity.

In lipid studies, absolute retention time (RT) variation during sample analyses is easily increased by small differences in experimental conditions (preparatory, chromatographic, and instrumental parameters). However, more repeatable separation techniques along with feature alignment enables more reliable identification with RTs in inter-laboratory studies in the future. [128] Since measurements of $\mathrm{m} / \mathrm{z}$ ratio are significantly less deviant (parameters affecting $\mathrm{m} / \mathrm{z}$ : ionization efficiency and mass spectrometric setup), single $\mathrm{m} / \mathrm{z}$, feature, and low-resolution spectra matching are the most used means for quick identification. [7] If needed, high resolution precursor and product ion spectra are usually obtained to achieve accurate identification between highly similar species. For further optimization of mass spectrometric identification, adduct formation and control of collision energy should also be studied and used. It is commonly agreed that adduct ions are formed mostly during ionization [9], [73], which is why lipid species appear at the same retention time in both pESI-MS and nESI-MS. Apart from protonated and deprotonated species, adding of millimolar concentrations of salts can be harnessed for signal enhancement [129]. Though at too high concentrations lipid signals can drastically be inhibited due to background noise caused by ion clusters [129]. Common ligand 
ions are acetate $\left(\mathrm{CH}_{3} \mathrm{COO}^{-}\right)$[56], [68], formate $\left(\mathrm{HCOO}^{-}\right)$[48], [50], [57], [58], [60], [75-77], and ammonium $\left(\mathrm{NH}_{4}{ }^{+}\right)[48],[50]$, [56-58], [60], [74-76]. Sodium $(\mathrm{Na}+)$ and potassium $(\mathrm{K}+)$ adducts are also commonly observed, though no controlled addition of such salts was noticed [92].

High resolution RP-HPLC separates structural isomers of lipid classes, [9] up to the point of carbon chain regioisomers (i.e. sn-positions of lipids). However, comprehensive identification of isobars and isomers could not be achieved by solely using MS/MS. For example, Lisa et al. [16] and Blaženović et al. [25] reported unresolved isomers and isobars with identical CNs and DBs but different fatty acyl composition. Adducts with same nominal mass can be separated with MS/MS [23], but specific information like sn-position of DBs needs other methods, like derivatizing of free fatty acids to form 4-iodobenzyl esters [130]. The reaction is made to esterified fatty acids into distinguishable epoxides via ketone dioxide catalyst and oxidant [131] or to oxetane-adducts via the Paternó-Büchi photochemical reaction. [132], [133], [134] Mass spectrometric solutions include second-generation fragmentation (MS/MS/MS) by dual stage collision-induced dissociation (CID) [135] or CID and ozone-induced dissociation combined, or silver-ion chromatography (currently achieved only with TGs) [81], [112], [136]. Recently, Zhao et al. [119] also mentioned electron impact excitation of ions from organics (EIEIO) and photo-ultraviolet dissociation (PUVD, photodissociation [130]) in the list of nonCID methods for sn and DB position. Since lyso-forms of GPs, i.e. sn-2 acyl lipids retain less in a HPLC column than their sn-1 isomers, they can be identified as separated double peaks. [9]

\subsection{Quantification of lipids}

Burla et al. [99] (a statement of the global lipidome community) compiled guideline recommendations for quantitative analysis in clinical sample matrices, specifically absolute quantification of lipids in plasma and serum. For the quick comparison of absolute 
quantification results, lipid concentrations should be reported in the SI-units mol/L or molar percentages whenever possible. [99]

In comparison to lipid identification, quantitative analysis is increasingly dependent on control samples as well as the quality and concentration of included standards, i.e. internal standards and calibrants. Common control samples include blanks and quality control (QC): Blanks are included for standard impurity monitoring and validation purposes, whereas QC samples (a pooled sample of all study samples in a batch) serves to monitor and correct batch-related uncertainty, instrumental errors and evaluate the performance of lipid analytes for validation purposes. [99]

Absolute quantitative calibration of lipids is either conducted via spiked QC samples (surrogate calibration) or by direct introduction of standards in the study sample as is often done in singlepoint and standard addition calibration. [75] However, single-point calibrations are reported to overestimate target lipid concentrations due to inherent flaws in the regression. [75] An ideal calibrant for complex matrices has an identical response factor, ionization efficiency and experiences comparable matrix effects as the analyte. [137]

For better understanding quantitative UHPLC-MS, some differences between HPLC-MS and DIMS should be pointed out. Though DIMS is more robust at the high-concentration lipids $(\mu \mathrm{mol} / \mathrm{L}-\mathrm{mmol} / \mathrm{L})$ of a sample, HPLC-MS is advantageous for the identification and quantification of low- and very low-abundance lipids (<nmol/L-mmol/L). [137], [99] Anyway, lipid class coverage and sensitivity are considerably improved in HPLC-MS experiments. [99] As an inherent disadvantage in HPLC-MS, the risk of lipid-lipid interaction increases due to the enrichment of same lipid species occurring in the column, though hetero-interaction between different lipid species decreases. [137] HPLC-MS uses peak areas in contrast to DIMS, where more stable ionization conditions enable quantification via peak intensity. [82] 
Moreover, the fluctuating quality and quantity of molecular species in the ion source is a recurring problem for lipid quantifications. These issues are amplified by the increasing complexity of experimental setups, such as chromatographic gradients affecting electrical properties (i.e. ionization efficiency) of the eluent. This has implications not only for the ionization efficiency, but also for the MS instrument's response factor: alongside solvent composition, Drotleff et al. [75] reports post-acquisition $\mathrm{MS}^{\mathrm{n}}$ procedures like SWATH or multiple reaction monitoring (MRM) to further deviate the detector's response values from one unit per molecule. Drotleff et al. [75] proposed "post-acquisition recalibration" as a reasonable compromise, if not enough sample is available for using the standard addition method. Then, sensitivities of analytes can be determined via a certified reference material. For lipids, only the NIST CRM 1950 plasma reference is currently acknowledged. [75] Standard mixtures, such as the Splash ${ }^{\circledR}$ Lipidomix $^{\circledR}$ mixture (Avanti Polar Lipids Inc., Alabaster, AL, USA) have been used for mimicking corresponding lipid concentrations in experiments as in biological fluids, e.g. human plasma, and for normalization of the analyses [14], [28], [66]. Since lipidome isotope labelling of yeast (LILY, complete lipidome carbon labelling with C13 isotope [138]) technology is discovered very recently, studies on using it in practice could not be considered in this review.

Furthermore, solvent-system dependent lipid concentrations of $>10-100 \mathrm{mmol} / \mathrm{L}$ are reported to form significant amounts of poorly ionic lipid aggregates (results found in a DIMS experiment). [137] The formation of aggregates like dimer, oligomer and micellar structures is further increased by the hydrophobicity of the lipid analyte and polarity of the mobile phase. [137]

Biological matrices include inherent variation in concentrations. UHPLC-MS experiments mostly revolve relative quantification and study changes in the lipidome of biological systems. However, in general HPLC-MS lipidomics has grown enough to advance further in absolute 
quantification of targeted lipids. [99] Main limitations of absolute quantification in HPLC-MS concern ambiguities preceding calibration, i.e. the structural versatility of lipid species, lipid concentrations at the lower (or upper) border of their linearity range, and various matrix effects such as ion suppression and enhancement. [99] Whereas polar lipid species quantification reaches a $\sim 5 \%$ accuracy (due to the polar headgroup predominantly explains their MS sensitivity) [137]. Mid- to nonpolar lipids like TGs and cholesteric esters are more effected by their specific structure in ESI-MS. [104] Thus, these lipids need more attention in standard representation. More details on quantitative and validation in lipid analyses are informed in Refs. [99] and [104].

\section{Data analysis of lipids}

According to the lipidome community, experimentally acquired raw data should be available for result-validation purposes and study re-analysis, whereas analytical details and results should be included in a file with acknowledged "XML[-based] or structured tabular format". [99] Corresponding raw data information can be taken from the proteomic field, which has practical formats for efficient data sharing. [99]

\subsection{Normalization}

Normalization of raw data is used to reduce systematic fluctuation concerning an accurate measurement. For example, Boysen et al. [139] developed the best matched internal standard normalization (B-MIS), an algorithm-driven solution for correcting non-biological variation of raw data (i.e. ion suppression by matrix effects, chromatographic quality and analytical drift). Obscuring variation, i.e. changes of peak area as a function of concentration, is compared between an unrestricted set of internal standards and analyte peaks in a QC sample. As a result, the algorithm chooses a suitable standard for normalization if the relative standard deviation of the peak area is improved by 40\%. [139] Furthermore, Drotleff et al. [76] considered B-MIS to be the best normalization model for reducing intragroup variation between the coefficient of 
variation, median absolute deviation and variance. His team observed a small difference in real positives favouring this normalization, but also noted that normalization models may work in a complementary fashion with their respective strengths and weaknesses. [76] Though not yet applied in absolute quantification experiments, B-MIS appears as a good alternative for modelbased peak area normalization in relative quantification methods. [139], [76]

Normalization is done with single standards representing the whole range of lipid classes. Suitable internal calibration standards with the same head groups and similar, but not identical fatty acids (e.g. odd-numbered $<1 \%$ abundant in higher organisms [104]), or isotope-labeled fatty acids are preferred for normalization [104]. The most used internal standards are deuterated at the carbon chain of their FA end to have either 7 or 9 deuterium atoms. [75] Internal standards included in QC samples are used for post-correction of systematic errors (i.e. drift and other batch-biases) [104], [99] like changes in peak shapes influencing the peak areas and for monitoring carry over [112].

\subsection{Data libraries}

Typically, lipid metabolite features in untargeted analyses are compared to available library spectra. [66], [118] Therefore, acquired data is represented by a "closest-match" for the $\mathrm{m} / \mathrm{z}$ or total ion spectrum that is compared, typically accompanied by a score from 1-100\%. Evidently, this approach may lead to false positive results, when the database used is incomplete or when the reference data is noisy [128].

Along with home-made databases [30], [32], [33], [36], [51], [54], [68], [78], commercial and open-source libraries have gained popularity and variety both for monitoring the lipid range with MS by characterizing fragmentation patterns for selective identity searching of lipids. Information about individual libraries and their respective types are compiled in Table 4. Specific tools like open-source softwares are mentioned in Table 5, whereas chemometric and statistical concepts are introduced in the next chapters. Additional information can be found 
from the LSI document (https://lipidomics-standards-initiative.org/links) and LIPID MAPS pages (http://lipidmaps.org/resources/tools/index.php).

\subsection{Chemometric tools}

Typically, metabolic correlation and significance analyses of lipids is conducted with a set of visual chemometric analyses and statistical methods. Chemometric methods provide an overview on analyte trends and outliers in relationship to their observations (features, spectra, etc.) with principal component analysis (PCA) [5], [7], [13], [16], [17-19], [27], [30], [31], [3336], [38-40], [43], [47-49], [51], [52], [54], [56-60], [62-64], [66], [68], [69], [72], [73], [7579], [124], systematic but hidden/uncorrelated variation between lipidome states with partial least-squares regression - discriminant analysis (PLS-DA) [3], [7], [10], [11], [26], [32], [34], [35], [48], [59], [60], [64], [76], [79], or orthogonal projection of latent structures discriminant analysis (OPLS-DA) [4], [6], [9], [19], [27], [33], [36], [37], [49], [50], [52], [54], [57], [60], [69], [78], [79], (relative or exact) concentration evaluation with boxplots [10], [18], [19], [32], [34], [37], [40], [44], [45], [48], [58], [60], [61], [67], [69], [73], [76] or a heatmap [3], [5], [7], [10], [11], [17], [26-28], [30-32], [34-38], [40-42], [45], [47], [48], [52], [56-58], [62], [68], [69], [73], [77], [79] visualizations for significant outlier detection such as the Bland-Altman plot [16], [40], [43-45], [55], analyte interaction or metabolic interlinkage of lipid species via hierarchical cluster analysis (HCA, often included with heatmap analysis) [3], [5], [7], [10], [17], [26], [27], [34], [37], [39], [40], [47-49], [68], [69], [73], [77], [79], interactive network [3], [26], [47] or pathway analysis [3], [7], [11], [26], [27], [37], [60], and diagnostic tools such as importance testing of PLS-DA variables with variable importance projection (VIP) [3], [10], [26], [33-37], [48], [50], [52], [57], [64], [79], OPLS-DA variable reliability and importance evaluation via S-plots [6], [27], [50], [57], [69], [79] or volcano plots [11], [31], [41], [42], [48], [60], [63], [67], [74], [77], [78], and method or sample comparisons with Venn diagrams [11], [17], [27], [30], [45], [49], [60], [66], [76]. 
PCA [140] pursues to flatten a large dataset with multiple variables to find the most important two sources of variation, i.e. principal components. This is done by comparing the transitions between all variables and flattening them into two dimensions with matrix operations. Thus, the most important information about multivariate data is found. This reduction process may help to divide the studied compounds into different groups and characterize them. [141] Similarly, PLS-DA seeks to flatten multivariate data to find the most fitting parallel twodimensional plane representing the whole dataset, [140] whereas OPLS-DA reduces the dataset to an orthogonal plane found with the partial least square plateau of the dataset. [142] Briefly, HCA clusters and categorizes sample components according to their apparent covariant relationship. [143]

Before actual multivariate data-analysis, the data needs often to be transformed, i.e. cleaned, scaled and re-centered, as is often done with e.g. statistical noise corrections or discarding of noisy data/spectra/outliers, unit variance or Pareto scaling and mean centering for PCA. [144] Chemometric methods, such as PCA, PLS-DA and OPLS-DA have kept their positions as the most common tools for visualizing analyte groupings (PCA) and metabolic changes (PLS-DA, OPLS-DA). Furthermore, VIP has emerged as an emphasis estimator of PLS-DA variables [3], [8], [26], [32], [34], [37], [64], [79] emphasizing the importance (i.e. magnitude) of each variable. In this context, lipids in the projection plot with VIP scores $>1$ are most accountable to predict changes in metabolism. More extensive analyses can be found in Ref. [88]

\subsection{Statistical tools}

In lipidomic research, statistical methods have developed into a broad variety of numerical tests and visualization techniques. Targeted and untargeted methods are two distinctively different approaches, as well as the means of data processing. Gorrochategui et al. [103] divided the targeted processing of metabolites into five phases: (1) the acquisition of raw data, (2) the contemplation of which database should be used when considering the research question and 
analytes, (3) the pre-processing and identification of these metabolites, (4) the normalization and quantification of the identified species, and (5) the reflection of the results on the biochemical/physiological context of metabolic pathways. [103] In contrast to targeted approaches, untargeted approaches need more careful experimental MS data processing, data modification, and evaluation for pre-processing to attain sufficient data quality. By this way, relevant biomarkers can be identified from the totality of detected features/peaks. The data quality is directly affected by the sensitivity and resolving power of the applied instrumentation. The non-targeted steps include the same pathway elucidation as in the targeted analyses, however, with respective appropriate tools. [103]

Statistical methods include a null hypothesis to test similarities by using one dataset. However, the $p$-value parameter in the Student's $t$-test [2], [10], [14], [15], [18], [19], [26], [27], [32], [37-39], [42], [50], [52], [54], [56], [57], [60], [61], [63], [67], [72], [79], the non-parametric Mann-Whitney $U$-test, [15], [31], [35], [41], [50], [57], [58], [74], [77], and the analysis of variance test (ANOVA) [2], [7], [10], [17], [22], [27], [32], [33], [46], [64], [69], [79] are for comparison of two or more datasets. These methods are often used for validation of analytical data and for detection of changes (e.g. analyte concentrations) between a control sample and authentic samples. Together with statistical tests, correction methods such as the BenjaminiHochberg test [145] are used to calculate the false discovery rate (FDR), i.e. minimizing false positive data in the dataset [56-58], [60], [74], [76].

The $t$-test designed for comparing two independent variables (e.g. patient versus control group lipidome data and that before and after drug intake) may be unreliable, when the sample size number is small $(\mathrm{n}<30)$ or when other kinds than normal distributions arise through the data processing [63]. This is the case for most sample number described in the articles reviewed for this paper. The average number of real samples studied was around 10-20 with the excluded outliers of 283 [36] and 10115 [94] test subjects. As to analyse control samples, there was a 
clear split of 7-16 and 30 in the number of controls used in the individual studies [30], [34]. Some cases of statistical testing of the datasets may be questionable, since e.g. $t$-tests were not reliable, when variances between populations were different. [146] For these kinds of cases, an unequal variance test like the Storey [74], [115] or Welch [48] $t$-test may be used instead [48], [74].

The Mann-Whitney U-test [147] (also known as the Wilcoxon rank-sum test) is used in mathematical data handling, when there are not any fixed parameters, which can be used to evaluate similarities of two independent dataset medians with a null hypothesis. For two sets with same or different sample sizes, the values of both datasets are sorted from the smallest to the largest one. The ranking integer is divided, if two values are identical: for instance, when two datapoints have the value 2 , they will be both noted as 1.5 [147]. Then, the separately ranked sample value sets are individually summed. When any of the sums reaches the critical value range of the $U$-test, the null hypothesis can be rejected. Akin to the Student's $t$-test [63], the $U$-test is evaluated by determining the $\mathrm{z}$-score $\left(z=\frac{x_{i}-\mu}{S D}\right.$, where $\mathrm{x}_{\mathrm{i}}$ is a value of a single datapoint, $\mu$ the population mean and SD the population standard deviation) from a normal distribution, suitable for a large number of studied samples (>30) [147]. In essence, the z-score is a distance-measurement of a single datapoint in relation to a normal distribution's standard deviation, determining the distance between its mean and single measurement.

A Mann-Whitney $U$-test [147] can be fitted for non-Gaussian distribution data. It is typically combined with a Benjamini-Hochberg (or Bonferroni-Holm [63]) test to exclude false positive values, thus providing the FDR. These methods were applied in multiple studies to limit uncertainty in the results of lipids. Furthermore, Paepe et al. [27] and Gong et al. [60] used cross-validated ANOVA test (CV-ANOVA) to improve reliability of the identified analytes. The reviewed papers also gave information that Mann-Whitney $U$-tests and FDR corrections 
with the Benjamini-Hochberg test [145] were used to discover the real positive results from analysed MS datasets.

In the Benjamini-Hochberg method [145] $p$-values of two sample sets (between the control and the test groups) are inflated mathematically to reveal possible false positive results. Since affected and unaffected lipids represent two distinct normal distributions, false positive values can be ideally seen to differentiate a distribution from the normal when using a big sample number in the study. In the data analysis of lipid species, the original $p$-values are inflated and excluded by their significance desired for the $p$-values (e.g. $p=0.1,10 \%$ significance). The $p$ values are then ranked from the smallest to the largest, after which the individual $p$-values are converted to a largest-to-smallest sequence. The largest $p$-value is kept, but the second largest $p$-value is determined as the smaller value of the two options. Therefore, either it is the same value preceding it, or it is the value calculated with a separate equation [145].

Lately, Tietz-Bogert et al. [31] calculated the FDR value in a lipido-metabolomic study by searching significant biomarkers of primary sclerosing cholangitis [31], which is a disorder of lipids in the bile duct of unknown origin. The concentrations of lipid species in control samples of healthy individual's and the clinical samples of sick patient's blood and bile were compared to find changes with a statistical significance of $p=0.01$ [31]. Simpler classical methods like ANOVA for multiple variables [2], [32], [46] were only occasionally used.

Machine learning and software advancements for multi-ionic identification have allowed to evaluate MS spectra produced with UHPLC-MS in silico, which is only demonstrated in a few recent articles within our scope [3], [18], [21], [25], [33], [67]. Due to machine learning techniques and their discovered use in omics, automated lipidomic analyses, receiver operating characteristic curve/area under curve (ROC/AUC) cross-validation analyses [3], [60], [67], [76] random forest studies [18], [32], [59], [67], neural network applications [67], [96], in silico spectra evaluation [21], and CCS value generation algorithms [25] have become in use. 


\section{Discussion}

UHPLC-MS lipidomics is divided into isotope-labelled standard methods and fully standardless algorithm-based analyses. Furthermore, the separation and acquisition of highly abundant lipid classes $(\mathrm{mmol} / \mathrm{L})$ from mid- $(\mu \mathrm{mol} / \mathrm{L})$ and low-abundance $(\leq \mathrm{nmol} / \mathrm{L})$ classes and their species is a challenge. [113] This is especially the case when (semi-)quantitative analysis is conducted for all quantifiable lipids in the sample, which can be the case for indepth studies on biological materials.

When comparing matrix effects in pharmacokinetic analysis made with HPLC and supercritical fluid chromatography (SFC) coupled with ESI-MS/MS, Svan et al. [148] observed a higher amount of ion suppression in SFC than in HPLC due to more ion enhancement. Is this general notion also applicable to HPLC-MS in lipidomics? Further information may be obtained from matrix effects via post-column infusion [148] as demonstrated by Drotleff et al. [75] or observations from more laborious (though more informative) post-extraction process. [149] Both of these tools may be important for improving the understanding of lipidome analysis in the future, as sightings of such matrix effect analysis is rare in recent lipid papers.

For practical reasons, a limitation of the comprehensive lipidome analysis is caused by the lipids without reasonable sensitivity. [9] Thus, the solution in that case is the Pareto principle, i.e. a limited number of biomolecules (lipid species) can be explained and measured using the main part of the studied biomass. [9] This is especially understandable in studies, where all the components are not relevant to the scientific question proposed. For example, metabolic lipid profiling with uncorrelated variation analysis (PLS-DA, OPLS-DA) in combination with metabolic knowledge highlight certain lipids from others to determine the most viable biomarkers. These biomarkers may form a conjoint array, which can already explain the changes in metabolism. 
The studies reviewed revealed a constant change, which affect retention time and peak focusing in a HPLC method: flow rate, gradient and oven temperature. Apart from an isocratic [43] and three-solvent eluents [10], [32], using aqueous - organic solvent gradients by increasing organic solvent percentage appeared in stepped (two [21], [40], [48] to three [25], [29], [38], [39] plateau steps or two [6], [69] to three [51] inclined steps), curved (exponential, [8], [37], [45], [67] logarithmic, [2], [19], [24], [41], [42], [50], [71] or s-curve [20], [27], [39], [49], [59], [63], [66]) and most popularly in linear [3], [9], [12], [13], [16], [17], [18], [22], [26], [28], [33], [34], [47], [52-54], [56], [67], [78] form. Modifications and combinations such as an inverted (i.e. increase in polar solvent percentage) linear [60], [79] or logarithmic [11] gradient, pyramidic [57], [77], pit-like [15], modified $S$-curve [5], [7], [45], [64] linear/isocratic [19], [23], exponential/linear [62], [68], [75], exponential/exponential [65], $S$-curve/exponential [64] and $S$-curve/isocratic [5] gradient were also used.

In particular, it was observed that the lipid studies were done under various column heating temperatures, from room temperature to $65{ }^{\circ} \mathrm{C}$. [65] Instrumentation for keeping the oven temperature stabile is very important in lipids analysis standardization. Furthermore, the research was done under various mobile phase temperatures, i.e. lipid analytes experienced significant temperature changes (up to $10-20{ }^{\circ} \mathrm{C}$ ) from column inlet to outlet. [107], [150] In addition, as heat is propagated from the column walls to the centre, temperature gradients are evident. [107] Though this is partly considered by narrow bore packed columns and the preequilibration of the analyses, the eluents flown from solvent units in room-temperature may cool down the column unevenly resulting in separation of lipids with low resolution.

The choose of column properties in lipid analyses should be considered by sorbent chemistry [109], particle size, pore size [86], and particle technology [117]. The unanimously preferred column (i.d. $2.1 \mathrm{~mm}$ ) is not necessarily optimal, since narrower columns are more prone to wall effects. [107], [144] This is due to a relatively more heterogeneous packing in comparison to 
bigger columns (internal diameter larger, i.d.). Moreover, a bigger i.d. (3 mm) is argued to enhance column efficiency and allow higher flow rates. [107] However, this may be an insignificant issue compared to the current disparity in lipidome method standardization and validation. [97]

Avanti Polar Lipids (https://avantilipids.com/tech-support/physical-properties/ionizationconstants) provides pKa-values of phospholipids to be closest to buffer pKa at 2.6 (PS phosphate group), 3 (PAs), 2.9-3.5 (PGs) and 5.5 (carboxyl group of PS) when compared to the pKa values of formic acid (3.74 [151]) and acetic acid (4.75 [151]). This means that when those organic acids are used PSs (and PAs/PGs at low $\mathrm{pH}$ ) are never once charged ions. Further problems may arise for amines such as in sphingosine (pKa 9.1 [152]), PE (9.6 [153]) or PS (9.8 [153]) when ammonia (pKa 9.25 [151]) is used. Modifiers used as eluent additives may also lead to fluctuations of $\mathrm{pH}$ in non-buffered systems in on-line coupled HPLC-MS. [154] The phenomena are possible either in the eluent during electrochemical ionization due to charge-balancing redox reactions, and during droplet shrinkage, since it may cause structural perturbations and discrepancy in the ratios of ionization species. [154]

In lipidome analytics, interdisciplinary analyses on multifunctional and computational methods will be crucial to effective and improved data processing. Furthermore, it is important to use the most practical parameters such as precursors, fragments, and CCS values in evaluation of data to get fast identification and determination of lipids. Multifunctional methods used are statistic and chemometric analyses, whereas computational methods include algorithms, dataprocessing interfaces, specific software, and machine learning strategies. Both method categories were attempted to be refined with tailored software platforms for necessary data preprocessing, pattern recognition, and for using large datasets with an immense amount of dataprocessing interfaces. 
Concerning common terminology, Aristizabal et al. [155] suggest names depending on the acquired structural information: brutto (e.g. PC 36:1), medio (e.g. PC(18:0/18:1), sn-position not known), genio (e.g. PC(18:0/18:1), sn-position known) and infinio (e.g. PC(18:0/18:1-9Z), sn-position not known). The same research group identified two distinct trends in lipidomics, which are the global analysis of lipids (coined macrolipidomics) and the specialized analyses for low-abundant lipids (microlipidomics).

Finally, the recognition correlation of HCA analyses is derived from information often visualized in the Heatmaps. Since HCA sorts out (i.e. clusters) groups of similar analyte species with each other, similar tools can potentially be developed by means of identifying and distinguishing matrices from each other.

As the scope of this review focuses mainly on UHPLC applications, it may limit some aspects of progress in HPLC-MS lipidomics as a whole. However, this was deemed necessary as publications on lipidomics have drastically increased. [156], [157] In our opinion -as may be representative for lipid studies in general- the most neglected topic mainly left out in the dataset was nutritional lipidomics [133] (mainly personalized nutrition [158] and nutritional intervention [159] studies). This is in tune with the main tendency of lipidomics towards lifesciences and bioanalytical questions concerning physiology and pathology. Furthermore, UHPLC-IMS-MS $^{\mathrm{n}}$ studies were rarer than might be expected from current trends in HPLCIMS-MS ${ }^{\mathrm{n}}$ lipidomics. Our dataset between the years 2017-2019 (topics discussed elsewhere [101]) included research on lipid method development, physiological profiling and metabolic changes via multicomponent analysis including drug development and biomarker studies. Sample matrices included plants, microbiota, mammals, fish and human patients. [101] As UHPLC instruments are less frequent in the total scheme of lipidomics compared to the commonly used HPLC instruments, limitations concerning this review's scope on lipidome applications must be noted. For instance, the use of acetone in an eluent mixture was only used 
by Castro-Gómez et al. [15] though some studies use a HPLC-MS ternary gradient separation in the fashion of Graeve et al. [160] ; acetone/ethyl acetate (v/v 2:1) and alkane:ethyl acetate (alkane: e.g. isooctane or $n$-heptane, v/v 99.8:0.2) is applied. [161] The lipidome community has also gone out of its way to develop a comprehensive and high-throughput method for lipid analysis, which puts emphasis on simple and quick sample preparation, high resolution/sensitivity $\mathrm{MS}^{\mathrm{n}}$ analysis and data handling via extensively automated data processing. Consequently, a derivatization step for UHPLC-MS was used only once in our dataset [8] where TMSD methylation was applied for separate acidic lipid analysis; neutral and basic lipids were directly analysed after sample preparation. [8] Though derivatization may be necessary for very low-abundance lipids or to increase lipid class specificity, another reason for the reduction of sample preparation steps is the minimization of experimental errors. [137] A particularly important outlier in lipidome applications was the research on double bond position elucidation; for the absolute determination of double bond positions, other options than multiple collision $\mathrm{MS}^{\mathrm{n}}$-experiments or spectral library matching have been developed.

\section{Conclusions}

Current challenges in lipidomics are closely related to the lack of uniform methods across the field. Compared with the lipid research generally made with RP-HPLC-MS, UHPLC-MS needs more focus on the chromatographic method parameters, such as stationary (e.g. column choice, oven temperature) and mobile phase properties (flow rate, $\mathrm{pH}$, solvents, gradients, eluent modifiers).

Normalization with representative or single internal standards has been widely adopted in the field. Since computational methods alongside chemometric and statistical methods have increased in both importance and usage in HPLC-MS technology for cross-validation and dataanalysis, lipidomics needs interdisciplinary studies to reach full potential with big and complex datasets. The process of typical data-analyses in lipidomics is suggested to be reduced into four 
parts: Pre-processing, identification/quantitation, chemometric, and statistical analyses. A substantial amount of specific processing tools are provided, i.e. commercial and open-source ones, including self-made algorithms. Since ever, more lipid libraries have emerged as well for untargeted, relatively targeted and in silico methods. Currently, chemometric analyses appear to be popular in lipidomics, i.e. for developing methods, profiling lipids in samples, and evaluating metabolic relationships between lipid species. For accurate identification, ion mobility mass spectrometry has been applied with liquid chromatography, alongside chromatographic separation modes with mass-spectrometric ionization, or/and $\mathrm{MS}^{\mathrm{n}}$ systems. When chemometric methods shed light to the inter- and intra-analyte properties of typically biological medium, statistical analyses were used for data-validation (e.g. significance testing and filtering out of false positives) and detection of abonormal changes (e.g. metabolic profiling of diseases).

Funding: This research received no external funding.

Conflicts of Interest: All authors declare no conflict of interest.

\section{References}

[1] Sumner, L. W., Amberg, A., Barrett, D., Beale, M. H., Beger, R., Daykin, C. A., Fan, T. W. M., Fiehn, O., Goodacre, R., Griffin, J. L., Hankemeier, T., Hardy, N., Harnly, J., Higashi, R., Kopka, J., Lane, A. N., Lindon, J. C., Marriott, P., Nicholls, A. W., Reily, M. D., Thaden, J. J., \& Viant, M. R. (2007). Proposed minimum reporting standards for chemical analysis. Metabolomics 3(3), 211-221.

[2] Liu, M. Y., Burgos, A., Ma, L., Zhang, Q., Tang, D., \& Ruan, J. (2017). Lipidomics analysis unravels the effect of nitrogen fertilization on lipid metabolism in tea plant (Camellia sinensis L.). BMC plant biology 17(1), 165, 1-10. 
[3] Wu, J., Wu, Q., Wang, D., Kong, J., Dai, W., Wang, X., \& Yu, X. (2017). Common lipid features of lethal ventricular tarchyarrhythmias (LVTAs) induced by myocardial infarction and myocardial ion channel diseases. Scientific reports 7(1), 4220, 1-11.

[4] Dong, S., Zhang, R., Liang, Y., Shi, J., Li, J., Shang, F., Mao, X., \& Sun, J. (2017).

Changes of myocardial lipidomics profiling in a rat model of diabetic cardiomyopathy using UPLC/Q-TOF/MS analysis. Diabetology \& metabolic syndrome 9(1), 56, 1-9.

[5] Van Meulebroek, L., De Paepe, E., Vercruysse, V., Pomian, B., Bos, S., Lapauw, B., \& Vanhaecke, L. (2017). Holistic lipidomics of the human gut phenotype using validated ultrahigh-performance liquid chromatography coupled to hybrid orbitrap mass spectrometry. Analytical chemistry 89(22), 12502-12510.

[6] Ferchaud-Roucher, V., Croyal, M., Moyon, T., Zair, Y., Krempf, M., \& Ouguerram, K. (2017). Plasma lipidome analysis by liquid chromatography-high resolution mass spectrometry and ion mobility of hypertriglyceridemic patients on extended-release nicotinic acid: a pilot study. Cardiovascular drugs and therapy 31(3), 269-279.

[7] Chao, H. C., Chen, G. Y., Hsu, L. C., Liao, H. W., Yang, S. Y., Wang, S. Y., Li, Y. L., Tang, S. C., Tseng, Y. J., \& Kuo, C. H. (2017). Using precursor ion scan of 184 with liquid chromatography-electrospray ionization-tandem mass spectrometry for concentration normalization in cellular lipidomic studies. Analytica chimica acta 971, 68-77.

[8] Lee, J. W., Mok, H. J., Lee, D. Y., Park, S. C., Kim, G. S., Lee, S. E., Lee, Y.S., Kim, K. P., \& Kim, H. D. (2017). UPLC-QqQ/MS-based lipidomics approach to characterize lipid alterations in inflammatory macrophages. Journal of proteome research 16(4), 1460-1469.

[9] Triebl, A., Trötzmüller, M., Hartler, J., Stojakovic, T., \& Köfeler, H. C. (2017).

Lipidomics by ultrahigh performance liquid chromatography-high resolution mass 
spectrometry and its application to complex biological samples. Journal of Chromatography B $1053,72-80$.

[10] Cheema, A. K., Mehta, K. Y., Fatanmi, O. O., Wise, S. Y., Hinzman, C. P., Wolff, J., \& Singh, V. K. (2018). A Metabolomic and lipidomic serum signature from nonhuman primates administered with a promising radiation countermeasure, gamma-tocotrienol. International journal of molecular sciences 19(1), 79, 1-16

[11] Chen, Y., Wen, S., Jiang, M., Zhu, Y., Ding, L., Shi, H., Dong, P., Yang, J., \& Yang, Y. (2017). Atherosclerotic dyslipidemia revealed by plasma lipidomics on ApoE-/- mice fed a high-fat diet. Atherosclerosis 262, 78-86.

[12] Li, Z., Guan, M., Lin, Y., Cui, X., Zhang, Y., Zhao, Z., \& Zhu, J. (2017). Aberrant lipid metabolism in hepatocellular carcinoma revealed by liver lipidomics. International journal of molecular sciences 18(12), 2550, 1-15.

[13] Ribeiro, M. A., Murgu, M., de Moraes Silva, V., Sawaya, A. C., Ribeiro, L. F., Justi, A., \& Meurer, E. C. (2017). The screening of organic matter in mineral and tap water by UHPLC-HRMS. Talanta 174, 581-586.

[14] Bang, G., Kim, Y. H., Yoon, J., Yu, Y. J., Chung, S., \& Kim, J. A. (2017). On-Chip Lipid Extraction Using Superabsorbent Polymers for Mass Spectrometry. Analytical [22] Li, Q., Liang, X., Zhao, L., Zhang, Z., Xue, X., Wang, K., \& Wu, L. (2017). UPLC-Q-exactive orbitrap/MS-based lipidomics approach to characterize lipid extracts from bee pollen and their in vitro anti-inflammatory properties. Journal of agricultural and food chemistry $65(32)$, $6848-6860$

[15] Castro-Gómez, P., Montero, O., \& Fontecha, J. (2017). In-depth lipidomic analysis of molecular species of triacylglycerides, diacylglycerides, glycerophospholipids, and 
sphingolipids of buttermilk by GC-MS/FID, HPLC-ELSD, and UPLC-QToF-MS. International journal of molecular sciences 18(3), 605, 1-20.

[16] Lísa, M., Cífková, E., Khalikova, M., Ovčačíková, M., \& Holčapek, M. (2017).

Lipidomic analysis of biological samples: comparison of liquid chromatography, supercritical fluid chromatography and direct infusion mass spectrometry methods. Journal of Chromatography A 1525, 96-108.

[17] Patterson, R. E., Kirpich, A. S., Koelmel, J. P., Kalavalapalli, S., Morse, A. M., Cusi, K., Sunny, K., McIntyre, L. M., Garrett, T. J., \& Yost, R. A. (2017). Improved experimental data processing for UHPLC-HRMS/MS lipidomics applied to nonalcoholic fatty liver disease. Journal of Chromatography B 1002, 260-266.

[18] Lloyd, A. J., Beckmann, M., Wilson, T., Tailliart, K., Allaway, D., \& Draper, J. (2017). Ultra high performance liquid chromatography-high resolution mass spectrometry plasma lipidomics can distinguish between canine breeds despite uncontrolled environmental variability and non-standardized diets. Metabolomics 13, 15, 1-11.

[19] Chang, W. Q., Zhou, J. L., Li, Y., Shi, Z. Q., Wang, L., Yang, J., Li, P., Liu, L. F., \& Xin, G. Z. (2017). An in vitro approach for lipolysis measurement using high-resolution mass spectrometry and partial least squares based analysis. Analytica chimica acta 950, 138-146. [20] Zhang, H., Gao, Y., Sun, J., Fan, S., Yao, X., Ran, X., Zheng, C., Huang, M. \& Bi, H. (2017). Optimization of lipid extraction and analytical protocols for UHPLC-ESI-HRMSbased lipidomic analysis of adherent mammalian cancer cells. Analytical and bioanalytical chemistry 409(22), 5349-5358.

[21] Witting, M., Ruttkies, C., Neumann, S., \& Schmitt-Kopplin, P. (2017). LipidFrag: Improving reliability of in silico fragmentation of lipids and application to the Caenorhabditis elegans lipidome. PloS one 12(3), e0172311, 1-23. 
[22] Li, Q., Liang, X., Zhao, L., Zhang, Z., Xue, X., Wang, K., \& Wu, L. (2017). UPLC-Qexactive orbitrap/MS-based lipidomics approach to characterize lipid extracts from bee pollen and their in vitro anti-inflammatory properties. Journal of agricultural and food chemistry 65(32), 6848-6860.

[23] Beccaria, M., Inferrera, V., Rigano, F., Gorynski, K., Purcaro, G., Pawliszyn, J., Dugo, P., \& Mondello, L. (2017). Highly informative multiclass profiling of lipids by ultra-high performance liquid chromatography-Low resolution (quadrupole) mass spectrometry by using electrospray ionization and atmospheric pressure chemical ionization interfaces. Journal of Chromatography A 1509, 69-82.

[24] Yang, J. S., Lee, J. C., Byeon, S. K., Rha, K. H., \& Moon, M. H. (2017). Size dependent lipidomic analysis of urinary exosomes from patients with prostate cancer by flow field-flow fractionation and nanoflow liquid chromatography-tandem mass spectrometry. Analytical chemistry 89(4), 2488-2496.

[25] Blaženović, I., Shen, T., Mehta, S. S., Kind, T., Ji, J., Piparo, M., Cacciola, F., Mondello, L., \& Fiehn, O. (2018). Increasing Compound Identification Rates in Untargeted Lipidomics Research with Liquid Chromatography Drift Time-Ion Mobility Mass Spectrometry. Analytical chemistry 90(18), 10758-10764.

[26] Wu, J., Wu, Q., Dai, W., Kong, J., Lv, J., Yu, X., Wang, X., \& Wang, D. (2018). Serum lipid feature and potential biomarkers of lethal ventricular tachyarrhythmia (LVTA) induced by myocardial ion channel diseases: a rat model study. International journal of legal medicine 132(2), 439-448.

[27] De Paepe, E., Van Meulebroek, L., Rombouts, C., Huysman, S., Verplanken, K., Lapauw, B., Wauters, J., Hemeryck, L. Y., Vanhaecke, L., \& Vanhaecke, L. (2018). A validated multi-matrix platform for metabolomic fingerprinting of human urine, feces and 
plasma using ultra-high performance liquid-chromatography coupled to hybrid orbitrap highresolution mass spectrometry. Analytica chimica acta 1033, 108-118.

[28] Manni, M. M., Sot, J., Arretxe, E., Gil-Redondo, R., Falcón-Pérez, J. M., Balgoma, D., Alonso, C., Goñi, F. M., \& Alonso, A. (2018). The fatty acids of sphingomyelins and ceramides in mammalian tissues and cultured cells: Biophysical and physiological implications. Chemistry and physics of lipids 217, 29-34.

[29] Yuan, Z. X., Majchrzak-Hong, S., Keyes, G. S., Iadarola, M. J., Mannes, A. J., \& Ramsden, C. E. (2018). Lipidomic profiling of targeted oxylipins with ultra-performance liquid chromatography-tandem mass spectrometry. Analytical and bioanalytical chemistry 410(23), 6009-6029.

[30] Xuan, Q., Hu, C., Yu, D., Wang, L., Zhou, Y., Zhao, X., Li, Q, Hou, X. \& Xu, G. (2018). Development of a High Coverage Pseudotargeted Lipidomics Method Based on Ultra-High Performance Liquid Chromatography-Mass Spectrometry. International Journal of Molecular Sciences 90(12), 7608-7616.

[31] Tietz-Bogert, P., Kim, M., Cheung, A., Tabibian, J., Heimbach, J., Rosen, C., Nandakumar, M., Lazaridis, K. N., LaRusso, N. F., Sung, J. \& O’Hara, S. (2018). Metabolomic Profiling of Portal Blood and Bile Reveals Metabolic Signatures of Primary Sclerosing Cholangitis. International journal of molecular sciences 19(10), 3188, 1-19.

[32] Cheema, A., Hinzman, C., Mehta, K., Hanlon, B., Garcia, M., Fatanmi, O., \& Singh, V. (2018). Plasma Derived Exosomal Biomarkers of Exposure to Ionizing Radiation in Nonhuman Primates, International journal of molecular sciences 19(11), 3427, 1-13.

[33] Chen, Y., Ma, Z., Shen, X., Li, L., Zhong, J., Min, L. S., Xu, L., Li, H., Zhang, J., \& Dai, L. (2018). Serum lipidomics profiling to identify biomarkers for non-small cell lung cancer, Atherosclerosis 262, 78-86. 
[34] Lee, H., Choi, J. M., Cho, J. Y., Kim, T. E., Lee, H. J., \& Jung, B. H. (2018). Regulation of endogenic metabolites by rosuvastatin in hyperlipidemia patients: An integration of metabolomics and lipidomics. Chemistry and physics of lipids 214, 69-83.

[35] Shan, J., Qian, W., Shen, C., Lin, L., Xie, T., Peng, L., Xu, J., Yang, R., Ji, J., \& Zhao, X. (2018). High-resolution lipidomics reveals dysregulation of lipid metabolism in respiratory syncytial virus pneumonia mice. RSC Advances 8(51), 29368-29377.

[36] Gao, X., Luo, J., Lü, L., Zhang, L., Zhang, S., \& Cui, J. (2018). RNA-Seq and UHPLCQ-TOF/MS Based Lipidomics Study in Lysiphlebia japonica. Scientific reports 8, 7802, 1-12. [37] Yan, B., Chu, H., Yang, D., Sze, K. H., Lai, P. M., Yuan, S., Shuai, H., Wang, Y., Kao, R. Y. T., Chan, J. F. W., \& Yuen, K. Y. (2019). Characterization of the Lipidomic Profile of Human Coronavirus-Infected Cells: Implications for Lipid Metabolism Remodeling upon Coronavirus Replication. Viruses 11(1), 73, 1-16.

[38] He, Z., Wang, Y., Zhang, Y., Cheng, H., \& Liu, X. (2018). Stereoselective bioaccumulation of chiral PCB 91 in earthworm and its metabolomic and lipidomic responses. Environmental pollution 238, 421-430.

[39] Law, T. H., Volk, H. A., Pan, Y., Zanghi, B., \& Want, E. J. (2018). Metabolic perturbations associated with the consumption of a ketogenic medium-chain TAG diet in dogs with idiopathic epilepsy. British Journal of Nutrition 120(5), 484-490.

[40] Chao, Y., Gao, S., Wang, X., Li, N., Zhao, H., Wen, X., Kou, Z., \& Dong, X. (2018). Untargeted lipidomics based on UPLC-QTOF-MS/MS and structural characterization reveals dramatic compositional changes in serum and renal lipids in mice with glyoxylate-induced nephrolithiasis, Journal of Chromatography B 1095, 258-266 
[41] Lee, J. H., Yang, J. S., Lee, S. H., \& Moon, M. H. (2018), Analysis of lipoproteinspecific lipids in patients with acute coronary syndrome by asymmetrical flow field-flow fractionation and nanoflow liquid chromatography-tandem mass spectrometry. Journal of Chromatography B 1099, 56-63.

[42] Kim, S. H., Yang, J. S., Lee, J. C., Lee, J. Y., Lee, J. Y., Kim, E., \& Moon, M. H. (2018). Lipidomic alterations in lipoproteins of patients with mild cognitive impairment and Alzheimer's disease by asymmetrical flow field-flow fractionation and nanoflow ultrahigh performance liquid chromatography-tandem mass spectrometry. Journal of Chromatography A $1568,91-100$.

[43] Gobo, L. A., de Carvalho, L. M., Temp, F., Viana, C., \& Mello, C. F. (2018). A rapid method for identification and quantification of prostaglandins in cerebral tissues by UHPLCESI-MS/MS for the lipidomic in vivo studies. Analytical biochemistry 545, 98-103.

[44] Drotleff, B., Hallschmid, M., \& Lämmerhofer, M. (2018). Quantification of steroid hormones in plasma using a surrogate calibrant approach and UHPLC-ESI-QTOF-MS/MS with SWATH-acquisition combined with untargeted profiling. Analytica chimica acta 1022, $70-80$.

[45] Danne-Rasche, N., Coman, C., \& Ahrends, R. (2018). Nano-LC/NSI MS refines lipidomics by enhancing lipid coverage, measurement sensitivity, and linear dynamic range. Analytical chemistry 90(13), 8093-8101.

[46] Ulmer, C. Z., Jones, C. M., Yost, R. A., Garrett, T. J., \& Bowden, J. A. (2018). Optimization of Folch, Bligh-Dyer, and Matyash sample-to-extraction solvent ratios for human plasma-based lipidomics studies. Analytica chimica acta 1037, 351-357.

[47] Jeucken, A., \& Brouwers, J. F. (2019). High-Throughput Screening of Lipidomic Adaptations in Cultured Cells, Biomolecules 9, 42, 1-14. 
[48] Marqueño, A., Blanco, M., Maceda-Veiga, A., \& Porte, C. (2019). Skeletal muscle lipidomics as a new tool to determine altered lipid homeostasis in fish exposed to urban and industrial wastewaters. Environmental science \& technology 53, 8416-8425.

[49] Rombouts, C., De Spiegeleer, M., Van Meulebroek, L., De Vos, W. H., \& Vanhaecke, L. (2019). Validated comprehensive metabolomics and lipidomics analysis of colon tissue and cell lines. Analytica Chimica Acta 1066, 79-92.

[50] Li, J., Gao, Y., Guan, L., Zhang, H., Chen, P., Gong, X., Li, D., Liang, X., Huang, M., \& Bi, H. (2019). Lipid profiling of peri-implantation endometrium in patients with premature progesterone rise in the late follicular phase. The Journal of Clinical Endocrinology \& Metabolism 104(11), 5555-5565.

[51] Goracci, L., Valeri, A., Sciabola, S., Aleo, M. D., Moritz, W., Lichtenberg, J., \& Cruciani, G. (2019). A novel lipidomics-based approach to evaluating the risk of clinical hepatotoxicity potential of drugs in 3D human microtissues. Chemical Research in Toxicology 33, 1, 258-270

[52] Wang, X., Xu, Y., Song, X., Jia, Q., Zhang, X., Qian, Y., \& Qiu, J. (2019). Analysis of glycerophospholipid metabolism after exposure to PCB153 in PC12 cells through targeted lipidomics by UHPLC-MS/MS. Ecotoxicology and environmental safety 169, 120-127.

[53] Wang, J., Zhang, L., Xiao, R., Li, Y., Liao, S., Zhang, Z., Yang, W., \& Liang, B. (2019). Plasma lipidomic signatures of spontaneous obese rhesus monkeys. Lipids in health and disease $18,8,1-11$.

[54] Lin, M., Wang, Z., Wang, D., Chen, X., \& Zhang, J. (2019). A mathematical modelassisted UHPLC-MS/MS method for global profiling and quantification of cholesteryl esters in hyperlipidemic golden hamsters. Analytical chemistry 91, 4504-4512. 
[55] Zalloua, P., Kadar, H., Hariri, E., Farraj, L. A., Brial, F., Hedjazi, L., Lay, A. L.,

Colleum A., Dubus, J., Touboul, D., Matsuda, F., Lathrop, M., Nicholson, J. K.,Dumas, M.

E. \& Matsuda, F. (2019). Untargeted Mass Spectrometry Lipidomics identifies correlation between serum sphingomyelins and plasma cholesterol. Lipids in health and disease 18, 38, $1-10$.

[56] Kappler, L., Hoene, M., Hu, C., von Toerne, C., Li, J., Bleher, D., Hoffmann, C., Böhm, A., Kollipara, L., Zischka, H., Königsrainer, A., Häring, H. U., Peter, A., Xu, G., Sickmann, A., Hauck, S. M., Weigert, C., \& Lehmann, R. (2019). Linking bioenergetic function of mitochondria to tissue-specific molecular fingerprints. American Journal of PhysiologyEndocrinology and Metabolism 317, E374-E387.

[57] Fu, K., Wang, C., Gao, Y., Fan, S., Zhang, H., Sun, J., Jiang, Y., Liu, C., Guan, L., Liu, J., Huang, M., \& Bi, H. (2019). Metabolomics and lipidomics reveal the effect of hepatic Vps33b deficiency on bile acids and lipids metabolism. Frontiers in Pharmacology 10, 276, $1-13$.

[58] Yang, R., Zhang, Y., Qian, W., Peng, L., Lin, L., Xu, J., Xie, T., Ji, J., \& Shan, J. (2019). Surfactant Lipidomics of Alveolar Lavage Fluid in Mice Based on Ultra-High-Performance Liquid Chromatography Coupled to Hybrid Quadrupole-Exactive Orbitrap Mass Spectrometry. Metabolites 9, 80, 1-14.

[59] Creydt, M., Vuralhan-Eckert, J., Fromm, J., \& Fischer, M. (2019). Effects of elevated $\mathrm{CO} 2$ concentration on leaves and berries of black elder (Sambucus nigra) using UHPLC-ESIQTOF-MS/MS and gas exchange measurements. Journal of plant physiology 234, 71-79.

[60] Gong, L. L., Yang, S., Zhang, W., Han, F. F., Lv, Y. L., Xuan, L. L., Liu, H., \& Liu, L. H. (2019). Discovery of Metabolite Profiles of Metabolic Syndrome Using Untargeted and 
Targeted LC-MS based Lipidomics Approach. Journal of pharmaceutical and biomedical analysis $117,112848,1-8$.

[61] Chico, Y., Abad-García, B., Ochoa, B., \& Martínez, M. J. (2019). Lipidomic data uncover extensive heterogeneity in phosphatidylcholine structural variants in HepG2 cells. Data in brief 27, 104608, 1-9.

[62] Gao, X., Liu, W., Mei, J., \& Xie, J. (2019). Quantitative Analysis of Cold Stress Inducing Lipidomic Changes in Shewanella putrefaciens Using UHPLC-ESI-MS/MS. Molecules 24(24), 4609, 1-13.

[63] Chamberlain, C. A., Hatch, M., \& Garrett, T. J. (2019). Metabolomic and lipidomic characterization of Oxalobacter formigenes strains HC1 and OxWR by UHPLC-HRMS. Analytical and bioanalytical chemistry 411, 4807-4818.

[64] Liu, H., Guo, X., Zhao, Q., Qin, Y., \& Zhang, J. (2020). Lipidomics analysis for identifying the geographical origin and lactation stage of goat milk. Food chemistry 309, $125765,1-7$.

[65] Su, M., Subbaraj, A. K., Fraser, K., Qi, X., Jia, H., Chen, W., Reis, M. G., Agnew, M., Day, L., Roy, N. C., \& Young, W. (2019). Lipidomics of Brain Tissues in Rats Fed Human Milk from Chinese Mothers or Commercial Infant Formula. Metabolites 9, 253, 1-19.

[66] Calderón, C., Sanwald, C., Schlotterbeck, J., Drotleff, B., \& Lämmerhofer, M. (2019). Comparison of simple monophasic versus classical biphasic extraction protocols for comprehensive UHPLC-MS/MS lipidomic analysis of Hela cells. Analytica chimica acta 1048, 66-74.

[67] Matorras, R., Martinez-Arranz, I., Arretxe, E., Iruarrizaga-Lejarreta, M., Corral, B., Ibañez-Perez, J., ... \& Alonso, C. (2019). The lipidome of endometrial fluid differs between 
implantative and non-implantative IVF cycles. Journal of Assisted Reproduction and Genetics 37(2), 385-394.

[68] Hinz, C., Liggi, S., Mocciaro, G., Jung, S., Induruwa, I., Pereira, M., Bryant, C. E., Meckelmann, S. W., O'Donnell, V. B., Farndale, R. W., Griffin, J. L., \& Fjeldsted, J. (2019). A Comprehensive UHPLC Ion Mobility Quadrupole Time-of-Flight Method for Profiling and Quantification of Eicosanoids, Other Oxylipins, and Fatty Acids. Analytical Chemistry, $8025-8035$.

[69] King, A., Baginski, M., Morikawa, Y., Rainville, P. D., Gethings, L. A., Wilson, I. D., \& Plumb, R. S. (2019). Application of a Novel Mass Spectral Data Acquisition Approach to Lipidomic Analysis of Liver Extracts from Sitaxentan-Treated Liver-Humanized PXB Mice. Journal of proteome research 18(11), 4055-4064.

[70] Gupta, A., Dorlhiac, G. F., \& Streets, A. M. (2019). Quantitative imaging of lipid droplets in single cells. Analyst, 144(3), 753-765. nanoflow liquid chromatography-tandem mass spectrometry. Analytical chemistry 89(4), 2488-2496.

[71] Lee, G. B., Lee, J. C., \& Moon, M. H. (2019). Plasma lipid profile comparison of five different cancers by nanoflow ultrahigh performance liquid chromatography-tandem mass spectrometry. Analytica Chimica Acta 1063, 117-126.

[72] Yang, J. S., Kim, J. Y., Lee, J. C., \& Moon, M. H. (2019). Investigation of lipidomic perturbations in oxidatively stressed subcellular organelles and exosomes by asymmetrical flow field-flow fractionation and nanoflow ultrahigh performance liquid chromatographytandem mass spectrometry. Analytica chimica acta 1073, 79-89.

[73] Schlotterbeck, J., Chatterjee, M., Gawaz, M., \& Lämmerhofer, M. (2019).

Comprehensive MS/MS profiling by UHPLC-ESI-QTOF-MS/MS using SWATH data- 
independent acquisition for the study of platelet lipidomes in coronary artery disease. Analytica chimica acta 1046, 1-15.

[74] Calderón, C., Rubarth, L., Cebo, M., Merfort, I., \& Lämmerhofer, M. (2019). Lipid Atlas of Keratinocytes and Betulin Effects on its Lipidome Profiled by Comprehensive UHPLC-MS/MS With Data Independent Acquisition Using Targeted Data Processing. Proteomics (Early View), 1900113, 1-17.

[75] Drotleff, B., Illison, J., Schlotterbeck, J., Lukowski, R., \& Lämmerhofer, M. (2019).

Comprehensive Lipidomics of Mouse Plasma using Class-Specific Surrogate Calibrants and SWATH Acquisition for Large-Scale Lipid Quantification in Untargeted Analysis. Analytica Chimica Acta 1022, 70-80.

[76] Drotleff, B., \& Lämmerhofer, M. (2019). Guidelines for selection of internal standardbased normalization strategies in untargeted lipidomic profiling by LC-HR-MS/MS. Analytical chemistry 91, 9836-9843.

[77] Shan, J., Qian, W., Kang, A., Peng, L., Xie, T., Lin, L., Di, L., Xiao, P., \& Zhou, W. (2019). Lipid profile perturbations in the plasma and lungs of mice with LPS-induced acute lung injury revealed by UHPLC-ESI-Q Exactive HF MS analysis. Journal of pharmaceutical and biomedical analysis 162, 242-248.

[78] Wang, L., Li, X., Liu, L., da Zhang, H., Zhang, Y., Chang, Y. H., \& Zhu, Q. P. (2019). Comparative lipidomics analysis of human, bovine and caprine milk by UHPLC-Q-TOF-MS. Food chemistry 310, 125865, 1-8.

[79] Deng, Y., Pan, M., Nie, H., Zheng, C., Tang, K., Zhang, Y., \& Yang, Q. (2019). Lipidomic Analysis of the Protective Effects of Shenling Baizhu San on Non-Alcoholic Fatty Liver Disease in Rats. Molecules 24, 3943, 1-17. 
[80] Gross, R. W. (2017). The evolution of lipidomics through space and time. Biochimica et Biophysica Acta (BBA)-Molecular and Cell Biology of Lipids 1862(8), 731-739.

[81] Holcapek, M., Liebisch, G., \& Ekroos, K. (2018). Lipidomic Analysis, Analytical Chemistry 90, 4249-4257.

[82] Yang, K., \& Han, X. (2016). Lipidomics: techniques, applications, and outcomes related to biomedical sciences. Trends in biochemical sciences 41(11), 954-969.

[83] Hyötyläinen, T., \& Orešič, M. (2015). Optimizing the lipidomics workflow for clinical studies_-practical considerations. Analytical and bioanalytical chemistry 407(17), 49734993.

[84] Jurowski, K., Kochan, K., Walczak, J., Barańska, M., Piekoszewski, W., \& Buszewski, B. (2017). Comprehensive review of trends and analytical strategies applied for biological samples preparation and storage in modern medical lipidomics: State of the art. TrAC Trends in Analytical Chemistry 86, 276-289.

[85] Tumanov, S., \& Kamphorst, J. J. (2017). Recent advances in expanding the coverage of the lipidome. Current opinion in biotechnology 43, 127-133.

[86] Criscuolo, A., Zeller, M., Cook, K., Angelidou, G., \& Fedorova, M. (2019). Rational selection of reverse phase columns for high throughput LC-MS lipidomics. Chemistry and physics of lipids 221, 120-127.

[87] Öztaş, Y., \& Boşgelmez, İ. (2017). An Introduction to Lipidomics: From Laboratory to Clinic. Acta Medica 48(1), 14-23.

[88] Checa, A., Bedia, C., \& Jaumot, J. (2015). Lipidomic data analysis: tutorial, practical guidelines and applications. Analytica chimica acta 885, 1-16 
[89] Wakil, S. J., Stoops, J. K., \& Joshi, V. C. (1983). Fatty acid synthesis and its regulation. Regulation of Fatty Acid Synthesis Annual review of biochemistry 52(1), 537-579, p. 569.

[90] Van Meer, G., Voelker, D. R., \& Feigenson, G. W. (2008). Membrane lipids: where they are and how they behave. Nature Reviews Molecular Cell Biology 9(2), 112-124.

[91] Dennis, E. A.: "LIPID MAPS Lipid Metabolics Tutorial, Fatty Acid Biosynthesis", Powerpoint, 2010, University of California, San Diego

[92] LIPID MAPS: Tutorials and Lectures on Lipids, Categories of Lipids. 02.10.2018 http://lipidmaps.org/resources/tutorials/lipid_tutorial.php\#FA

[93] Fahy, E., Subramaniam, S., Brown, H. A., Glass, C. K., Merrill Jr, A. H., Murphy, R. C., ... \& Shimizu, T. (2005). A comprehensive classification system for lipids. European journal of lipid science and technology 107(5), 337-364.

[94] LIPID MAPS: Classification, nomenclature and structure drawing. 20.11.2018 https://www.lipidmaps.org/resources/tutorials/lipid_cns.html [95] Kim, H. S., Waqued, S. C., Nodurft, D. T., Devarenne, T. P., Yakovlev, V. V., \& Han, A. (2017). Raman spectroscopy compatible PDMS droplet microfluidic culture and analysis platform towards on-chip lipidomics. Analyst 142(7), 1054-1060.

[96] Koelmel, J. P., Kroeger, N. M., Ulmer, C. Z., Bowden, J. A., Patterson, R. E., Cochran, J. A., Beecher, C. W. W., Garrett, T. J.\& Yost, R. A. (2017). LipidMatch: an automated workflow for rule-based lipid identification using untargeted high-resolution tandem mass spectrometry data. BMC bioinformatics 18(1), 331, 1-11.

[97] Lipidomics Standards Initiative Consortium. (2019). Lipidomics needs more standardization. Nature Metabolism 1(8), 745-747. 
[98] Ovčačíková, M., Lísa, M., Cífková, E., \& Holčapek, M. (2016). Retention behavior of lipids in reversed-phase ultrahigh-performance liquid chromatography-electrospray ionization mass spectrometry. Journal of Chromatography A 1450, 76-85.

[99] Burla, B., Arita, M., Arita, M., Bendt, A. K., Cazenave-Gassiot, A., Dennis, E. A., Ekroos, K., Han, X., Ikeda, K., Liebisch, G., Lin, M. K., Loh, T. P., Meikle, P. J., Orešič, M., Quehenberger, O., Shevchenko, A., Torta, F., Wakelam, M. J. O., Wheelock, C. E., \& Wenk, M. R. (2018). MS-based lipidomics of human blood plasma: a community-initiated position paper to develop accepted guidelines. Journal of lipid research 59(10), 2001-2017. [100] Rodriguez-Mozaz, S., de Alda, M. J. L., \& Barceló, D. (2007). Advantages and limitations of on-line solid phase extraction coupled to liquid chromatography-mass spectrometry technologies versus biosensors for monitoring of emerging contaminants in water. Journal of chromatography A 1152(1-2), 97-115.

[101] Avela, H. F. \& Sirén, H. (2020) Advances in lipidomics, Clinica Chimica Acta. (in-print) [102] Kitov, P. I., Han, L., Kitova, E. N., \& Klassen, J. S. (2019). Sliding window adduct removal method (SWARM) for enhanced electrospray ionization mass spectrometry binding data. Journal of The American Society for Mass Spectrometry 30(8), 1446-1454.

[103] Gorrochategui, E., Jaumot, J., Lacorte, S., \& Tauler, R. (2016). Data analysis strategies for targeted and untargeted LC-MS metabolomic studies: Overview and workflow. TrAC Trends in Analytical Chemistry 82, 425-442.

[104] Lam, S. M., Tian, H., \& Shui, G. (2017). Lipidomics, en route to accurate quantitation. Biochimica et Biophysica Acta (BBA)-Molecular and Cell Biology of Lipids 1862(8), 752761. 
[105] Vu, N., Narvaez-Rivas, M., Chen, G. Y., Rewers, M. J., \& Zhang, Q. (2019). Accurate mass and retention time library of serum lipids for type 1 diabetes research. Analytical and bioanalytical chemistry 411(23), 5937-5949.

[106] Bielow, C., Mastrobuoni, G. Orioli, M., \& Kempa, S. (2017). On mass ambiguities in high-resolution shotgun lipidomics. Analytical chemistry, 89(5), 2986-2994.

[107] Dong, M. W. (2013), Myths in ultrahigh-pressure liquid chromatography, LCGC North America 31(10), 868-880

[108] Avela, H. F. (2019). Recent Developments and Challenges in Chromatographic Lipidomics. Helsinki University, E-thesis

[109] Cífková, E., Hájek, R., Lísa, M., \& HolLapek, M. (2016). Hydrophilic interaction liquid chromatography mass spectrometry of (lyso) phosphatidic acids,(lyso) phosphatidylserines and other lipid classes. Journal of Chromatography A 1439, 65-73. [110] Moldoveanu, S. C., \& David, V. (2016). Selection of the HPLC method in chemical analysis. Elsevier, ISBN:978-0-12-803684-6, 40-42

[111] Brouwers, J. F. (2011). Liquid chromatographic-mass spectrometric analysis of phospholipids. Chromatography, ionization and quantification. Biochimica et Biophysica Acta (BBA)-Molecular and Cell Biology of Lipids 1811(11), 763-775.

[112] Cajka, T., \& Fiehn, O. (2014). Comprehensive analysis of lipids in biological systems by liquid chromatography-mass spectrometry. TrAC Trends in Analytical Chemistry 61, 192206.

[113] Triebl, A., Trötzmüller, M., Eberl, A., Hanel, P., Hartler, J., \& Köfeler, H. C. (2014). Quantitation of phosphatidic acid and lysophosphatidic acid molecular species using hydrophilic interaction liquid chromatography coupled to electrospray ionization high resolution mass spectrometry. Journal of chromatography A 1347, 104-110. 
[114] Rozing, G. (2003). Trends in HPLC column formats-Microbore, nanobore and smaller. LC GC EUROPE 16(6 A), 14-19.

[115] Storey, J. D. (2003). The positive false discovery rate: a Bayesian interpretation and the q-value. The Annals of Statistics 31(6), 2013-2035.

[116] Mannur, V. S., Patel, D., Mastiholimath, V. S., \& Shah, G. (2011). Selection of buffers in LC-MS/MS: an overview. International Journal of Pharmaceutical Science s Review and Research 6(1), Article-008, 35-37.

[117] Cajka, T., \& Fiehn, O. (2016). Toward merging untargeted and targeted methods in mass spectrometry-based metabolomics and lipidomics. Analytical chemistry 88(1), 524-545.

[118] Monnin, C., Ramrup, P., Daigle-Young, C., \& Vuckovic, D. (2018). Improving negative liquid chromatography/electrospray ionization mass spectrometry lipidomic analysis of human plasma using acetic acid as a mobile-phase additive. Rapid Communications in Mass Spectrometry 32(3), 201-211.

[119] Zhao, X., Zhang, W., Zhang, D., Liu, X., Cao, W., Chen, Q., Ouyang, Z., \& Xia, Y. (2019). A lipidomic workflow capable of resolving sn-and C [double bond, length as m-dash] C location isomers of phosphatidylcholines. Chemical Science 10(46), 10740-10748.

[120] Xiao, Y.J.; Schwartz, B.; Washington, M.; Kennedy, A.; Webster, K.; Belinson, J.; Xu, Y. (2001). Electrospray ionization mass spectrometry analysis of lysophospholipids in human ascitic fluids: Comparison of the lysophospholipid contents in malignant vs. nonmalignant ascitic fluids. Analytical Biochemistry 290, 302-313.

[121] Lee, D. Y., Kind, T., Yoon, Y. R., Fiehn, O., \& Liu, K. H. (2014). Comparative evaluation of extraction methods for simultaneous mass-spectrometric analysis of complex 
lipids and primary metabolites from human blood plasma. Analytical and bioanalytical chemistry 406(28), 7275-7286.

[122] Taylor, N., White, T., \& Viant, M. (2017). Defining the baseline and oxidant perturbed lipidomic profiles of daphnia magna. Metabolites 7, 11, 1-14.

[123] Byeon, S. K., Kim, J. Y., Lee, J. Y., Chung, B. C., Seo, H. S., \& Moon, M. H. (2015). Top-down and bottom-up lipidomic analysis of rabbit lipoproteins under different metabolic conditions using flow field-flow fractionation, nanoflow liquid chromatography and mass spectrometry. Journal of Chromatography A 1405, 140-148.

[124] Zhang, J., Rector, J., Lin, J. Q., Young, J. H., Sans, M., Katta, N., Giese, N., Yu, W., Nagi, C., Suliburk, J., Liu, J., Bensussan, A.m DeHoog, R. J., Garza, K. Y., Ludolph, B., Sorace, A. G.,, Syed, A., Zahedivash, A., Milner, T. E., \& Eberlin, L. S. (2017). Nondestructive tissue analysis for ex vivo and in vivo cancer diagnosis using a handheld mass spectrometry system. Science translational medicine 9(406), eaan3968.

[125] Law, K. P., \& Zhang, C. L. (2019). Current Progress and Future Trends of Mass Spectrometry-based Archaeal Lipidomics. Organic geochemistry 134, 45-61.

[126] Vasilopoulou, C. G., Sulek, K., Brunner, A. D., Meitei, N. S., Schweiger-Hufnagel, U., Meyer, S. W., Barsch, A., Mann, M. \& Meier, F. (2020). Trapped ion mobility spectrometry and PASEF enable in-depth lipidomics from minimal sample amounts. Nature Communications, 11(1), 1-11.

[127] Sethi, S., \& Brietzke, E. (2017). Recent advances in lipidomics: Analytical and clinical perspectives. Prostaglandins \& other lipid mediators 128, 8-16.

[128] Smith, R., Mathis, A. D., Ventura, D., \& Prince, J. T. (2014). Proteomics, lipidomics, metabolomics: a mass spectrometry tutorial from a computer scientist's point of view. BMC Bioinformatics 15(Suppl 7), S9, 1-14. 
[129] Cech, N. B., \& Enke, C. G. (2001). Practical implications of some recent studies in electrospray ionization fundamentals. Mass spectrometry reviews, 20(6), 362-387.

[130] Pham, H. T., Trevitt, A. J., Mitchell, T. W., \& Blanksby, S. J. (2013). Rapid differentiation of isomeric lipids by photodissociation mass spectrometry of fatty acid derivatives. Rapid Communications in Mass Spectrometry 27(7), 805-815.

[131] Song, C., Gao, D., Li, S., Liu, L., Chen, X., \& Jiang, Y. (2019). Determination and quantification of fatty acid $\mathrm{C}=\mathrm{C}$ isomers by epoxidation reaction and liquid chromatographymass spectrometry. Analytica Chimica Acta 1086, 82-89.

[132] Murphy, R. C., Okuno, T., Johnson, C. A., \& Barkley, R. M. (2017). Determination of double bond positions in polyunsaturated fatty acids using the photochemical Paterno-Buchi reaction with acetone and tandem mass spectrometry. Analytical chemistry 89(16), 85458553.

[133] Smilowitz, J. T., Zivkovic, A. M., Wan, Y. J. Y., Watkins, S. M., Nording, M. L., Hammock, B. D., \& German, J. B. (2013). Nutritional lipidomics: molecular metabolism, analytics, and diagnostics. Molecular nutrition \& food research, 57(8), 1319-1335.

[134] Deng, J., Yang, Y., Liu, Y., Fang, L., Lin, L., \& Luan, T. (2019). Coupling PaternòBüchi Reaction with Surface-Coated Probe Nanoelectrospray Ionization Mass Spectrometry for In Vivo and Microscale Profiling of Lipid C $=\mathrm{C}$ Location Isomers in Complex Biological Tissues. Analytical chemistry 91(7), 4592-4599.

[135] Castro-Perez, J., Roddy, T. P., Nibbering, N. M., Shah, V., McLaren, D. G., Previs, S., Attygalle, A. B., Herath, K., Chen, Z., Wang, S.-P., Mitnaul, L., Hubbard, B. K., Vreeken, R. J., Johns, D. G., \& Hankemeier, T. (2011). Localization of fatty acyl and double bond positions in phosphatidylcholines using a dual stage CID fragmentation coupled with ion mobility mass spectrometry. Journal of the American Society for Mass Spectrometry, 22(9), 1552-1567. 
[136] Lísa, M., Netušilová, K., Franěk, L., Dvořáková, H., Vrkoslav, V., \& Holčapek, M. (2011). Characterization of fatty acid and triacylglycerol composition in animal fats using silver-ion and non-aqueous reversed-phase high-performance liquid chromatography/mass spectrometry and gas chromatography/flame ionization detection. Journal of Chromatography A 1218(42), 7499-7510.

[137] Yang, K., \& Han, X. (2011). Accurate quantification of lipid species by electrospray ionization mass spectrometry — meets a key challenge in lipidomics. Metabolites, 1(1), 21-40. [138] Rampler, E., Coman, C., Hermann, G., Sickmann, A., Ahrends, R., \& Koellensperger, G. (2017). LILY-lipidome isotope labeling of yeast: in vivo synthesis of 13 C labeled reference lipids for quantification by mass spectrometry. Analyst 142(11), 1891-1899.

[139] Boysen, A. K., Heal, K. R., Carlson, L. T., \& Ingalls, A. E. (2018). Best-matched internal standard normalization in liquid chromatography-mass spectrometry metabolomics applied to environmental samples. Analytical chemistry, 90(2), 1363-1369.

[140] Lennart Eriksson, Sartorius Stedim Biotech, Umetrics Suite blog - "OPLS vs PCA: Explaining differences or grouping data? “ https://blog.umetrics.com/explaining-differencesor-grouping-data-opls-da-vs-pca-data-analysis, page checked 24.06.2019

[141] Kelechi, A. C. (2012). Regression and principal component analysis: comparison using few regressors. American Journal of Mathematics and Statistics 2(1), 1-5.

[142] Wold, S., Ruhe, A., Wold, H., \& Dunn, III, W. J. (1984). The collinearity problem in linear regression. The partial least squares (PLS) approach to generalized inverses. SIAM Journal on Scientific and Statistical Computing 5(3), 735-743.

[143] Holme, P., Huss, M., \& Jeong, H. (2003). Subnetwork hierarchies of biochemical pathways. Bioinformatics 19(4), 532-538. 
[144] Kahler, E. G. F. T. (2016). Effects of Column Inner Diameter and Packed Bed [145] Benjamini, Y., \& Hochberg, Y. (1995). Controlling the false discovery rate: a practical and powerful approach to multiple testing. Journal of the Royal statistical society: series B (Methodological) 57(1), 289-300.

[146] Ruxton, G. D. (2006). The unequal variance t-test is an underused alternative to Student's t-test and the Mann-Whitney U test. Behavioral Ecology 17(4), 688-690.

[147] Nachar, N. (2008). The Mann-Whitney U: A test for assessing whether two independent samples come from the same distribution. Tutorials in quantitative Methods for Psychology 4(1), 13-20.

[148] Svan, A., Hedeland, M., Arvidsson, T., \& Pettersson, C. E. (2018). The differences in matrix effect between supercritical fluid chromatography and reversed phase liquid chromatography coupled to ESI/MS. Analytica chimica acta 1000, 163-171.

[149] Gergov, M., Nenonen, T., Ojanperä, I., \& Ketola, R. A. (2015). Compensation of matrix effects in a standard addition method for metformin in postmortem blood using liquid chromatography-electrospray-tandem mass spectrometry. Journal of analytical toxicology, 39(5), 359-364.

[150] Dolan, J. W. (2002). The importance of temperature. LC GC NORTH AMERICA 20(6), 524-531.

[151] Dolan, J. (2016). A guide to HPLC and LC-MS buffer selection. ACE HPLC Columnsultra inert base-deactivated HPLC columns, Advanced Chromatography Technologies (Aberdeen, Scotland), 1-20.

[152] Sasaki, H., Arai, H., Cocco, M. J., \& White, S. H. (2009). pH dependence of sphingosine aggregation. Biophysical journal 96(7), 2727-2733. 
[153] Tsui, F. C., Ojcius, D. M., \& Hubbell, W. L. (1986). The intrinsic pKa values for phosphatidylserine and phosphatidylethanolamine in phosphatidylcholine host bilayers. Biophysical journal 49(2), 459-468.

[154] Konermann, L. (2017). Addressing a common misconception: Ammonium acetate as neutral $\mathrm{pH}$ "buffer" for native electrospray mass spectrometry. Journal of the American Society for Mass Spectrometry 28, 1827-1835.

[155] Aristizabal, H. J. J., Bradley, R. M., Duncan, R. E., \& Stark, K. D. (2018) Categorizing and qualifying nutritional lipidomic data: defining brutto, medio, genio and infinio lipid species within macrolipidomics and microlipidomics. Current Opinion in Clinical Nutrition and Metabolic Care 21(5), 352-359.

[156] Holčapek, M., Liebisch, G., \& Ekroos, K. (2020). Lipidomic analysis. Analytical and Bioanalytical Chemistry 412, 2187-2189.

[157] Touboul, D., \& Ollero, M. (2019). Lipidomics Conquers a Niche, Consolidates Growth. International Journal Molecular Sciences 20(13), 3188, 1-4.

[158] Ibáñez, C., Mouhid, L., Reglero, G., \& Ramírez de Molina, A. (2017). Lipidomics insights in health and nutritional intervention studies. Journal of agricultural and food chemistry, 65(36), 7827-7842.

[159] de Castro, M. L., \& Quiles-Zafra, R. (2020). Lipidomics: an omics discipline with a key role in nutrition. Talanta 219, article 121197.

[160] Graeve, M., \& Janssen, D. (2009). Improved separation and quantification of neutral and polar lipid classes by HPLC-ELSD using a monolithic silica phase: application to exceptional marine lipids. Journal of Chromatography B 877(20-21), 1815-1819.

[161] Imbert, L., Gaudin, M., Libong, D., Touboul, D., Abreu, S., Loiseau, P. M., ... \& Chaminade, P. (2012). Comparison of electrospray ionization, atmospheric pressure chemical 
ionization and atmospheric pressure photoionization for a lipidomic analysis of Leishmania donovani. Journal of Chromatography A 1242, 75-83. 
Supplementary Table S1. Experimental conditions for the solid and mobile phase in chromatography

\begin{tabular}{|c|c|c|c|c|c|c|c|}
\hline Precolumn & Column & Polar Solvent (v/v ratios) & Organic Solvent (v/v ratios) & Solvent C & Additives & Year & Citation \\
\hline \multirow[t]{26}{*}{ Yes } & T3 C18 & 1:1 MeOH: $\mathrm{H}_{2} \mathrm{O}(\mathrm{pH} 7.5)$ (PG;PS) & 6:4 MeOH:ACN (GP;PS) & & AmFo, FoA & 2017 & {$[15]$} \\
\hline & C18 HSS & 19:19:2 ACN:MeOH: $\mathrm{H}_{2} \mathrm{O}$ & IPA & & AmFo, FoA & 2017 & [8] \\
\hline & C18 HSS T3 & 3:4:3 ACN:acetone:IPA (DG;TG) & 3:7 ACN:IPA (DG;TG) & & $\mathrm{AmOH}$ & 2017 & {$[15]$} \\
\hline & C18 BEH & $40: 60 \mathrm{ACN}: \mathrm{H}_{2} \mathrm{O}$ & 90:10 IPA:ACN & & AmAc, AcA & 2017 & [19] \\
\hline & $\mathrm{N} / \mathrm{A}$ & 40:60 ACN: $\mathrm{H}_{2} \mathrm{O}$ & 90:10 IPA:ACN & & AmAc & 2017 & {$[18]$} \\
\hline & $\mathrm{C} 18$ & $50: 50 \mathrm{ACN}: \mathrm{H}_{2} \mathrm{O}$ & 95:5 IPA:ACN & & AmFo, FoA & 2017 & {$[20]$} \\
\hline & $\mathrm{C} 18 \mathrm{CSH}$ & $\mathrm{H}_{2} \mathrm{O}$ & 50:50 IPA:ACN & & AmFo, FoA & 2017 & {$[11]$} \\
\hline & $\mathrm{C} 18$ & $60: 40 \mathrm{MeOH} / \mathrm{H}_{2} \mathrm{O}$ & 60:40 MeOH:IPA & & AmAc, AcA & 2017 & [7] \\
\hline & $\mathrm{C} 18$ & $60: 40 \mathrm{ACN}: \mathrm{H}_{2} \mathrm{O}$ & 90:10 IPA:ACN & & AmAc & 2017 & {$[22]$} \\
\hline & C18 & $60: 40 \mathrm{ACN}: \mathrm{H}_{2} \mathrm{O}$ & 90:10 IPA:ACN & & AmFo, FoA & 2017 & {$[21]$} \\
\hline & $\mathrm{C} 18 \mathrm{CSH}$ & $60: 40 \mathrm{ACN}: \mathrm{H}_{2} \mathrm{O}$ & 90:10 IPA:ACN & & - & 2017 & [4] \\
\hline & $\mathrm{C} 18 \mathrm{CSH}$ & $60: 40 \mathrm{ACN}: \mathrm{H}_{2} \mathrm{O}$ & 90:10 IPA:ACN & & AmAc, FoA & 2017 & {$[6]$} \\
\hline & $\mathrm{C} 18 \mathrm{CSH}$ & $60: 40 \mathrm{ACN}: \mathrm{H}_{2} \mathrm{O}$ & $90: 10$ IPA:ACN & & AmAc & 2017 & {$[22]$} \\
\hline & $\mathrm{C} 18 \mathrm{CSH}$ & $60: 40 \mathrm{ACN}: \mathrm{H}_{2} \mathrm{O}$ & 90:10 IPA:ACN & & AmFo, FoA & 2017 & {$[14]$} \\
\hline & C18 HSS & $60: 40 \mathrm{ACN}: \mathrm{H}_{2} \mathrm{O}$ & 90:10 IPA:ACN & & AmFo & 2017 & [3] \\
\hline & C18 BEH & $90: 10 \mathrm{ACN}: \mathrm{H}_{2} \mathrm{O}$ & $90: 80: 1$ IPA:ACN: $\mathrm{H}_{2} \mathrm{O}$ & & AmFo, FoA & 2017 & {$[17]$} \\
\hline & C18 BEH* & $90: 10 \mathrm{H}_{2} \mathrm{O}: \mathrm{ACN}$ & 20:20:60 MeOH:ACN:IPA & & AmFo, FoA & 2017 & {$[24]^{*}$} \\
\hline & C18 HSS T3 & 90:10 IPA:ACN & $30: 70 \mathrm{H}_{2} \mathrm{O}: \mathrm{ACN}$ & & AmFo & 2017 & {$[12]$} \\
\hline & HILIC, BEH & $\mathrm{H}_{2} \mathrm{O}$ & $96: 4 \mathrm{ACN}: \mathrm{H}_{2} \mathrm{O}$ & & AmAc & 2017 & [16] \\
\hline & $\mathrm{C} 8 \mathrm{BEH}$ & $\mathrm{H}_{2} \mathrm{O}$ & 2:5 IPA:ACN & & AmFo, FoA & 2017 & [9] \\
\hline & C18 BEH & $\mathrm{H}_{2} \mathrm{O}$ & 50:50 IPA:ACN & & - & 2017 & [19] \\
\hline & $\mathrm{C} 18^{* *}$ & $\mathrm{H}_{2} \mathrm{O}$ & 60:36:4 IPA:ACN: $\mathrm{H}_{2} \mathrm{O}$ & & AmFo & 2017 & {$[23]^{* *}$} \\
\hline & $\mathrm{C} 8$ & $\mathrm{H}_{2} \mathrm{O}$ & 70:30 ACN:IPA & & AmAc, FoA & 2017 & {$[2]$} \\
\hline & C18 BEH & $\mathrm{H}_{2} \mathrm{O}$ & $\mathrm{ACN}$ & 90:10 IPA:ACN & FoA & 2017 & [10] \\
\hline & C18 HSS T3 & $\mathrm{H}_{2} \mathrm{O}$ & $\mathrm{ACN}$ & & FoA & 2017 & [13] \\
\hline & C18 BEH & $\mathrm{H}_{2} \mathrm{O}$ & $\mathrm{MeOH}$ & & AmAc & 2017 & {$[5]$} \\
\hline
\end{tabular}

*nano-LC, **APCl instead of ESI, ***narrow-bore UHPLC

BEH: ethylene bridged hybrid, CSH: charged surface hybrid, HSS: high strength silica, SB: stable bond packing 


\begin{tabular}{|c|c|c|c|c|c|c|c|}
\hline Precolumn & Column & Polar Solvent (v/v ratios) & Organic Solvent (v/v ratios) & Solvent C & Additives & Year & Citation \\
\hline & $\mathrm{C} 18 \mathrm{CSH}$ & 40:60 ACN: $\mathrm{H}_{2} \mathrm{O}$ & 90:10 IPA:ACN & & AmFo, FoA & 2018 & {$[35]$} \\
\hline & C18 & 45:55 ACN: $\mathrm{H}_{2} \mathrm{O}$ & & & AcA & 2018 & [43] \\
\hline & C18 BEH & $60: 40$ ACN: $\mathrm{H}_{2} \mathrm{O}$ & $81: 10: 9$ IPA:ACN: $\mathrm{H}_{2} \mathrm{O}$ & & AmFo, FoA & 2018 & {$[40]$} \\
\hline & $\mathrm{C} 18 \mathrm{CSH}$ & $60: 40$ ACN: $\mathrm{H}_{2} \mathrm{O}$ & 90:10 ACN:IPA & & AmFo, FoA & 2018 & [39] \\
\hline & C18 & $60: 40$ ACN: $\mathrm{H}_{2} \mathrm{O}$ & 90:10 IPA:ACN & & AmFo & 2018 & [33] \\
\hline & C18 & $60: 40$ ACN: $\mathrm{H}_{2} \mathrm{O}$ & 90:10 IPA:ACN & & $A m A c$ & 2018 & [38] \\
\hline \multirow[t]{2}{*}{ Yes } & C18 CSH & $60: 40 \mathrm{ACN}: \mathrm{H}_{2} \mathrm{O}$ & 90:10 IPA:ACN & & AmFo, FoA & 2018 & {$[25]$} \\
\hline & C18 HSS & $60: 40$ ACN: $\mathrm{H}_{2} \mathrm{O}$ & 90:10 IPA:ACN & & AmFo & 2018 & {$[26]$} \\
\hline Yes & C18* & $60: 40$ ACN: $\mathrm{H}_{2} \mathrm{O}$ & 90:10 IPA:ACN & & AmFo, FoA & 2018 & {$[45]^{*}$} \\
\hline \multirow[t]{5}{*}{ Yes } & $\mathrm{C} 18^{* * *}$ & $60: 40$ ACN: $\mathrm{H}_{2} \mathrm{O}$ & 90:10 IPA:ACN & & AmFo, FoA & 2018 & {$[45]^{* * *}$} \\
\hline & C8 BEH & $60: 40$ ACN: $\mathrm{H}_{2} \mathrm{O}$ & 90:10 IPA:ACN & & AmAc & 2018 & [30] \\
\hline & C8 BEH & $60: 40$ ACN: $\mathrm{H}_{2} \mathrm{O}$ & 90:10 IPA:ACN & & AmAc & 2018 & {$[30]$} \\
\hline & C18 BEH & $60: 40$ ACN: $\mathrm{H}_{2} \mathrm{O}$ & 90:10 IPA:ACN & & AmFo & 2018 & [28] \\
\hline & C18 BEH & $60: 40$ ACN: $\mathrm{H}_{2} \mathrm{O}$ & $90: 8: 2$ IPA:ACN: $\mathrm{H}_{2} \mathrm{O}$ & & AmFo, FoA & 2018 & [46] \\
\hline Yes & C18 BEH* & $90: 10 \mathrm{H}_{2} \mathrm{O}: \mathrm{ACN}$ & 20:20:60 MeOH:ACN:IPA & & AmFo, FoA & 2018 & {$[41]^{*}$} \\
\hline \multirow[t]{12}{*}{ Yes } & C18 BEH* & $90: 10 \mathrm{H}_{2} \mathrm{O}: \mathrm{ACN}$ & 20:20:60 MeOH:ACN:IPA & & AmFo, FoA & 2018 & {$[42]^{*}$} \\
\hline & C18 SB & $\mathrm{H}_{2} \mathrm{O}$ & $90: 10 \mathrm{ACN}: \mathrm{H}_{2} \mathrm{O}$ & & AmAc, AcA & 2018 & [29] \\
\hline & $\mathrm{C} 18^{* *}$ & $\mathrm{H}_{2} \mathrm{O}$ & ACN & & FoA & 2018 & {$[44]^{* *}$} \\
\hline & C18 BEH & $\mathrm{H}_{2} \mathrm{O}$ & $\mathrm{ACN}$ & $90: 10$ IPA:ACN & FoA & 2018 & [32] \\
\hline & C18 BEH & $\mathrm{H}_{2} \mathrm{O}$ & $\mathrm{ACN}$ & & AcA & 2018 & [37] \\
\hline & C18 HSS & $\mathrm{H}_{2} \mathrm{O}$ & $\mathrm{ACN}$ & & FoA & 2018 & {$[27]$} \\
\hline & Amide & $\mathrm{H}_{2} \mathrm{O}$ & $\mathrm{MeOH}$ & & $\mathrm{AmHCO} 3$ & 2018 & [31] \\
\hline & Amide & $\mathrm{H}_{2} \mathrm{O}$ & $\mathrm{MeOH}$ & & AmFo & 2018 & [31] \\
\hline & C18 BEH & $\mathrm{H}_{2} \mathrm{O}$ & $\mathrm{MeOH}$ & & PFPA, FoA & 2018 & [31] \\
\hline & C18 BEH & $\mathrm{H}_{2} \mathrm{O}$ & $\mathrm{MeOH}$ & & PFPA, FoA & 2018 & [31] \\
\hline & $\mathrm{C} 18 \mathrm{CSH}$ & $\mathrm{H}_{2} \mathrm{O}$ & $\mathrm{MeOH}$ & & FoA & 2018 & [34] \\
\hline & C18 HSS & $\mathrm{H}_{2} \mathrm{O}$ & $\mathrm{MeOH}$ & & FoA & 2018 & [39] \\
\hline
\end{tabular}

*nano-LC, ${ }^{* *}$ APCl instead of ESI, ***narrow-bore UHPLC

BEH: ethylene bridged hybrid, CSH: charged surface hybrid, HSS: high strength silica, SB: stable bond packing 


\begin{tabular}{|c|c|c|c|c|c|c|c|}
\hline Precolumn & Column & Polar Solvent ( $\mathrm{v} / \mathrm{v}$ ratios) & Organic Solvent (v/v ratios) & Solvent C & Additives & Year & Citation \\
\hline & C18 BEH* & 10:90 $\mathrm{H}_{2} \mathrm{O}: \mathrm{ACN}$ & 20:20:60 MeOH:ACN:IPA & & AmFo, NH3 & 2019 & [71] \\
\hline & $\mathrm{C} 18 \mathrm{CSH}$ & $40: 60 \mathrm{ACN}: \mathrm{H}_{2} \mathrm{O}$ & 90:10 IPA:ACN & & AmFo, FoA & 2019 & [77] \\
\hline & C18 & $50: 50 \mathrm{ACN}: \mathrm{H}_{2} \mathrm{O}$ & 20:80 IPA:MeOH & & $A m A c$ & 2019 & {$[52]$} \\
\hline & C18 & 50:50 H2O:ACN & 5:95 ACN:IPA & & AmFo, FoA & 2019 & {$[50]$} \\
\hline & C18 & 50:50 H2O:ACN & 5:95 ACN:IPA & & AmFo, FoA & 2019 & [57] \\
\hline \multirow[t]{3}{*}{ Yes } & $\mathrm{C} 18 \mathrm{CSH}$ & $60: 40 \mathrm{ACN}: \mathrm{H}_{2} \mathrm{O}$ & $90: 10 \mathrm{ACN}: \mathrm{H}_{2} \mathrm{O}$ & & AmFo, FoA & 2019 & [55] \\
\hline & C18 HSS T3 & 60:40 ACN:H2O & 90:10 ACN:IPA & & AmFo, FoA & 2019 & {$[60]$} \\
\hline & $\mathrm{C} 18 \mathrm{CSH}$ & $60: 40$ ACN:H2O & 90:10 ACN:IPA & & AmFo, FoA & 2019 & {$[60]$} \\
\hline \multirow[t]{10}{*}{ Yes } & $\mathrm{C} 18 \mathrm{CSH}$ & $60: 40 \mathrm{ACN}: \mathrm{H}_{2} \mathrm{O}$ & 90:10 IPA:ACN & & AmFo, FoA & 2019 & {$[66]$} \\
\hline & $\mathrm{C} 18 \mathrm{CSH}$ & $60: 40$ ACN:H2O & 90:10 IPA:ACN & & AmFo, FoA & 2019 & [74] \\
\hline & $\mathrm{C} 8$ & $60: 40$ ACN:H2O & 90:10 IPA:ACN & & $A m A c$ & 2019 & {$[56]$} \\
\hline & $\mathrm{C} 18 \mathrm{CSH}$ & $60: 40$ ACN:H2O & 90:10 IPA:ACN & & AmFo, FoA & 2019 & {$[65]$} \\
\hline & $\mathrm{C} 18 \mathrm{CSH}$ & 60:40 ACN:H2O & 90:10 IPA:ACN & & AmFo & 2019 & [79] \\
\hline & C18 & $60: 40$ ACN:H2O & 90:10 IPA:ACN & & AmFo & 2019 & [78] \\
\hline & $\mathrm{C} 18 \mathrm{CSH}$ & $60: 40$ ACN:H2O & $90: 10$ IPA:ACN & & AmFo, FoA & 2019 & [69] \\
\hline & $\mathrm{C} 18 \mathrm{CSH}$ & $60: 40$ ACN:H2O & 90:10 IPA:ACN & & AmFo & 2019 & {$[62]$} \\
\hline & $\mathrm{C} 18 \mathrm{CSH}$ & 60:40 ACN:H2O & 90:10 IPA:ACN & & AmAc & 2020 & [64] \\
\hline & $\mathrm{C} 18 \mathrm{CSH}$ & $60: 40$ ACN:H2O & 90:10 IPA:ACN & & AmAc & 2020 & {$[64]$} \\
\hline Yes & C18 BEH & $60: 40 \mathrm{ACN}: \mathrm{H}_{2} \mathrm{O}$ & $90: 8: 2$ IPA:ACN: $\mathrm{H}_{2} \mathrm{O}$ & & AmFo, FoA & 2019 & {$[63]$} \\
\hline Yes & $\mathrm{C} 18 \mathrm{CSH}$ & 60:40 ACN:H2O & 90:9:1 IPA:ACN:H2O & & AmFo, FoA & 2019 & [75] \\
\hline \multirow[t]{6}{*}{ Yes } & $\mathrm{C} 18 \mathrm{CSH}$ & $60: 40$ ACN:H2O & 90:9:1 IPA:ACN:H2O & & AmFo, FoA & 2019 & [76] \\
\hline & $\mathrm{C} 18 \mathrm{CSH}$ & 60:40 H2O:ACN & 90:10 IPA:ACN & & AmFo, FoA & 2019 & {$[58]$} \\
\hline & HILIC & $70: 30 \mathrm{ACN}: \mathrm{H}_{2} \mathrm{O}$ & 90:10 ACN:acetone & & AmFo, FoA & 2019 & [47] \\
\hline & HILIC** & $70: 30 \mathrm{ACN}: \mathrm{H}_{2} \mathrm{O}$ & 90:10 ACN:acetone & & AmFo, FoA & 2019 & {$[47]^{* *}$} \\
\hline & C18 BEH & $\mathrm{ACN}: \mathrm{H} 2 \mathrm{O}$ & IPA:ACN & & AmFo & 2019 & [67] \\
\hline & $\mathrm{C} 18 \mathrm{CSH}$ & $\mathrm{H} 2 \mathrm{O}$ & 16:3 ACN:MeOH & & AcA & 2019 & [68] \\
\hline
\end{tabular}

*nano-LC, ${ }^{* *} \mathrm{APCl}$ instead of ESI, ***narrow-bore UHPLC

BEH: ethylene bridged hybrid, CSH: charged surface hybrid, HSS: high strength silica, SB: stable bond packing 


\begin{tabular}{|c|c|c|c|c|c|c|c|}
\hline Precolumn & Column & Polar Solvent ( $v / v$ ratios) & Organic Solvent (v/v ratios) & Solvent C & Additives & Year & Citation \\
\hline \multirow[t]{7}{*}{ Yes } & $\mathrm{C} 8$ & $\mathrm{H}_{2} \mathrm{O}$ & 55:40:5 ACN:IPA: $\mathrm{H}_{2} \mathrm{O}$ & & $A m A c$ & 2019 & {$[68]$} \\
\hline & C18 & $\mathrm{H}_{2} \mathrm{O}$ & 75:25 IPA:ACN & & AmFo & 2019 & [59] \\
\hline & C18 & $\mathrm{H}_{2} \mathrm{O}$ & 75:25 IPA:ACN & & AmAc & 2019 & [59] \\
\hline & C18 BEH & $\mathrm{H} 2 \mathrm{O}$ & $\mathrm{ACN}$ & & FoA & 2019 & [67] \\
\hline & F5 & $\mathrm{H} 2 \mathrm{O}$ & IPA & & AmFo, FoA & 2019 & {$[51]$} \\
\hline & C18 & $\mathrm{H}_{2} \mathrm{O}$ & $\mathrm{MeOH}$ & & AmAc, FoA & 2019 & {$[53]$} \\
\hline & C18 & $\mathrm{H}_{2} \mathrm{O}$ & $\mathrm{MeOH}$ & & AmAc, FoA & 2019 & [53] \\
\hline \multirow[t]{4}{*}{ Yes } & Phenyl & $\mathrm{H}_{2} \mathrm{O}$ & $\mathrm{MeOH}$ & & $A m A c$ & 2019 & [49] \\
\hline & C8 BEH & $\mathrm{H} 2 \mathrm{O}$ & $\mathrm{MeOH}$ & & AmFo, FoA & 2019 & {$[48]$} \\
\hline & C18 BEH & $\mathrm{MeOH}$ & 2:5 ACN:IPA & & AmAc, FoA & 2019 & {$[54]$} \\
\hline & C18 HSS & N/A & $\mathrm{N} / \mathrm{A}$ & & $\mathrm{N} / \mathrm{A}$ & 2019 & [61] \\
\hline
\end{tabular}

*nano-LC, **APCl instead of ESI, ***narrow-bore UHPLC

BEH: ethylene bridged hybrid, CSH: charged surface hybrid, HSS: high strength silica, SB: stable bond packing 


\section{Highlights}

- Method development and application enhancements in lipidomics

- The review sums up chemometric and statistical methods for current lipidomics

- State of the art data collection and evaluation is discussed

- Identification/quantitation of biological lipids

- Tandem MS data-independent and data-dependent analysis 


\title{
Advances in analytical tools and current statistical methods used in ultra-high- performance liquid chromatography-mass spectrometry of glycero-, glycerophospho- and sphingolipids
}

\section{Correspondence}

Henri F. Avela (MSc), Faculty of Science, Department of Chemistry, University of Helsinki, A.I. Virtasen Aukio 1 (P.O. Box 55), FI-00014 University of Helsinki, Finland; Heli Sirén (Assoc. Professor, Doc, PhD), Faculty of Science, Department of Chemistry, University of Helsinki, A.I. Virtasen Aukio 1 (P.O. Box 55), FI-00014 University of Helsinki, Finland, heli.m.siren@helsinki.fi

\begin{abstract}
The review concentrates on the properties of analytical and statistical ultrahigh-performance liquid chromatographic (UHPLC) - mass spectrometric (MS) methods suitable for glycero-, glycerophospho- and sphingolipids in lipidomics published between the years 2017-2019. Trends and fluctuations of conventional and nano-UHPLC methods with MS and tandem MS detection were observed in context of analysis conditions and tools used for data-analysis.

Whereas general workflow characteristics are agreed upon, more details related to the chromatographic methodology (i.e. stationary and mobile phase conditions) need evidently agreements. Lipid quantitation relies upon isotope-labelled standards in targeted analyses and fully standardless algorithm-based untargeted analyses. Furthermore, a wide spectrum of setups have shown potential for the elucidation of complex and large datasets by minimizing the risks of systematic misinterpretation like false positives. This kind of evaluation was shown to have increased importance and usage for cross-validation and data-analysis.
\end{abstract}

\section{Keywords}

Lipidomics, mass spectrometry, ultrahigh performance liquid chromatography, nano-liquid chromatography, chemometrics, statistical methods, multicomponent analysis

\section{Highlights}

- Method development and application enhancements in lipidomics

- The review sums up chemometric and statistical methods for current lipidomics

- State of the art data collection and evaluation is discussed

- Identification/quantitation of biological lipids

- Tandem MS data-independent and data-dependent analysis Figures included: 4 Tables included: 5

Pages of text: 23 Supplementary data: Supplementary data (3 pages), Table S1 (4 pages) 


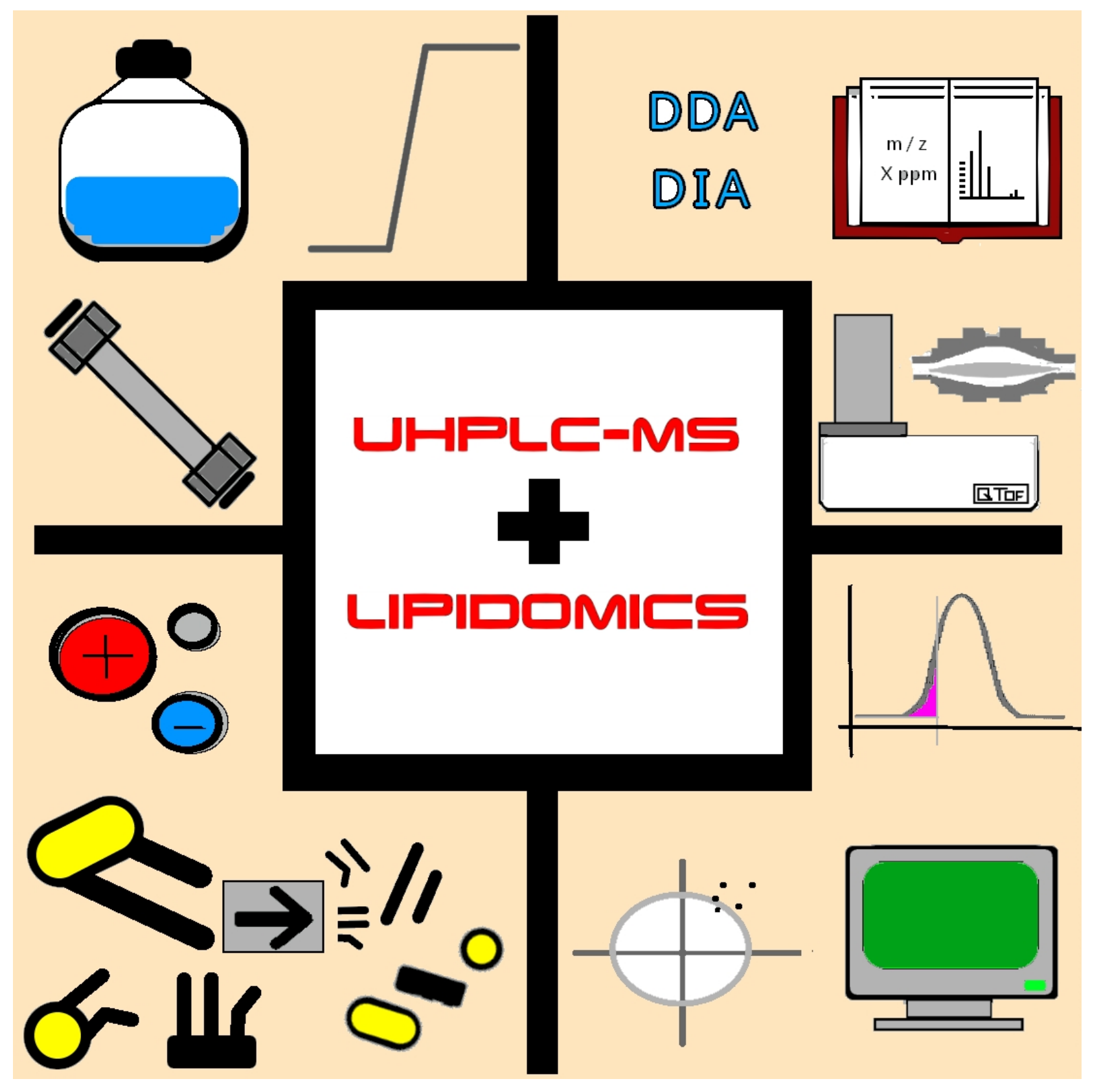




\title{
Advances in analytical tools and current statistical methods used in ultra-high- performance liquid chromatography-mass spectrometry of glycero-, glycerophospho- and sphingolipids
}

Henri F. Avela and Heli Sirén

Department of Chemistry, University of Helsinki, P.O. Box 55, FI-00014 University of Helsinki (FI-00560 Helsinki), Finland

Correspondence: Henri F. Avela (MSc), Faculty of Science, Department of Chemistry, University of Helsinki, A.I. Virtasen Aukio 1 (P.O. Box 55), FI-00014 University of Helsinki, Finland; Heli Sirén (Assoc. Professor, Doc, PhD), Faculty of Science, Department of Chemistry, University of Helsinki, A.I. Virtasen Aukio 1 (P.O. Box 55), FI-00014 University of Helsinki, Finland, heli.m.siren@helsinki.fi

\begin{abstract}
The review concentrates on the properties of analytical and statistical ultrahigh-performance liquid chromatographic (UHPLC) - mass spectrometric (MS) methods suitable for glycero-, glycerophospho- and sphingolipids in lipidomics published between the years 2017-2019. Trends and fluctuations of conventional and nano-UHPLC methods with MS and tandem MS detection were observed in context of analysis conditions and tools used for data-analysis.

Whereas general workflow characteristics are agreed upon, more details related to the chromatographic methodology (i.e. stationary and mobile phase conditions) need evidently agreements. Lipid quantitation relies upon isotope-labelled standards in targeted analyses and fully standardless algorithm-based untargeted analyses. Furthermore, a wide spectrum of setups have shown potential for the elucidation of complex and large datasets by minimizing the risks of systematic misinterpretation like false positives. This kind of evaluation was shown to have increased importance and usage for cross-validation and data-analysis.
\end{abstract}

\section{Keywords}

Lipidomics, mass spectrometry, ultrahigh performance liquid chromatography, nano-liquid chromatography, chemometrics, statistical methods, multicomponent analysis 


\section{Highlights}

- Method development and application enhancements in lipidomics

- The review sums up chemometric and statistical methods for current lipidomics

- State of the art data collection and evaluation is discussed

- Identification/quantitation of biological lipids

- Tandem MS data-independent and data-dependent analysis

\section{Abbreviations}

ACP acyl carrier protein, AF4 asymmetric flow field flow fractionation, ANOVA analysis of variance, AUC area under curve, CARS coherent anti-Stokes Raman scattering, CCS collision cross-section, CN total carbon, CRS coherent Raman scattering, DB double bond, DDA data dependent acquisition, DESI desorption electrospray ionization, DG diacylglycerol, DIA data independent acquisition, DIMS direct infusion MS, FDR false discovery rate, FA fatty acyl, FFA free fatty acid, GC gas chromatography, GL glycerolipid, GP glycerophospholipid, HCA hierarchical cluster analysis, i.d. internal diameter, IMS ion mobility spectrometry, HPLC liquid chromatography, LESA liquid extraction surface analysis, m/z mass-to-charge [ratio], LSI Lipidomic Standards Initiative, HR/LRMS high/low resolution mass spectrometry, MS all ions scans, MS/MS tandem mass spectrometry, MS/MS/MS second-generation fragmentation mass spectrometry $\mathrm{MS}^{\mathrm{n}}$ on-line coupled mass spectrometry system, MSI mass spectrometric imaging, m/z mass-to-charge ratio, NP normal phase, NSI nano-electrospray ionization, nESI negative ion mode in electrospray ionization, OPLS-DA orthogonal projections to latent structures discriminant analysis, (L)PA (lyso-)phosphatidic acid, (L)PC (lyso-)phosphatidylcholine, (L)PE lyso-phosphatidylethanolamine, pESI positive ion mode in electrospray ionization, (L)PG (lyso-) phospatidylglycerol, (L)PI (lyso-)phosphatidylinositol, NP-HPLC normal phase liquid chromatography, PIS precursor ion scan, PLS-DA partial least squares discriminant analysis, PRM parallel reaction monitoring, (L)PS (lyso- 
)phosphatidylserine, QqQ triple quadrupole mass analyzer, QTOF quadrupole - time of flight, ROC receiver operating characteristics curve, RP-HPLC reversed phase liquid chromatography, RT retention time, SP sphingolipid, SFC supercritical fluid chromatography, SWATH sequential window acquisition of all theoretical fragment ion mass spectra, SWARM sliding window adduct removal method, TG triacylglycerol, TOF time of flight mass analyzer, UHPLC ultra-high performance liquid chromatography, VIP variable importance projection

\section{Introduction}

Recent lipid research has emerged due to improved multidimensional computer algorithms and highly efficient commercial, open-source and in-house software platforms. Furthermore, data libraries for automated routine searching of mass spectra is adopted for lipid identification. International organizations have started together to harmonize knowledge about lipidomics. Especially, the Lipidomics Standard Initiative (LSI, https://lipidomics-standardsinitiative.org/) found under the International Lipidomic Society (https://lipidomicssociety.org/about/) and Lipid Home (https://www.lipidhome.co.uk/) strive to standardize lipidomic information with the globally acknowledged platform Lipid MAPS (https://www.lipidmaps.org/). Furthermore, Sumner et al. [1] have stated minimal requirements for retraceable lipid analyses, [1] which are important for integrated research in lipidomics. This review on lipid investigatios encompasses research conducted with ultra-high performance mass spectrometry (UHPLC-MS) during 2017-2019 [2-79]. To clarify, this review uses the term high performance liquid chromatography (HPLC) to describe both conventional HPLC and UHPLC. However, blindspots of exclusive UHPLC-research are attempted to be covered in the Discussion-section.

Particularly, half of the reviewed studies deal with computerized platforms to identify lipid species of several classes. These computerized platforms have successfully enabled data handling with in silico analyses, commonly generalized as machine learning algorithms. After 
all, the most popular means of comprehensive identification in -omics research is on-line coupled mass spectrometry systems (MS ${ }^{\mathrm{n}}$, e.g. tandem mass spectrometry [MS/MS]), which demand extensive and thorough data-handling. In the field of lipidomics, $\mathrm{MS}^{\mathrm{n}}$ is often divided into data-dependent acquisition (DDA) and data-independent acquisition (DIA). Here, DDA is typically defined as fragmentation of only targeted precursors, as is the case in parallel reaction monitoring (PRM) [15], [44], [45]. It is a technique, in which all fragments of the chosen lipid precursors are measured. In DIA, all available lipid precursors are fragmented and measured in a full scan with a set of collision energy (CE) or energies, detecting a substantial amount of lipid adducts fragmentation patterns, e.g. in an all ions scan $\left(\mathrm{MS}^{\mathrm{E}}\right)$ [9-15], [32-34], [55-61], [68], [74].

Recent DIA research were applied in a novel MS technique called sequential window acquisition of all theoretical fragment ions spectra (SWATH MS) [6], [44], [73-76] and similarly, with QTOF-specific SONAR technology [69]. The consecutive fragmentation or scan of precursors (i.e. PRM for DDA and SWATH or SONAR for DIA) and their product ions provide cleaner MS spectra in favour of library searches, an improved detection rate, broadened range, and expanded specificity in any given fragmentation frame compared to other methods [34], [44], [73], [69]. Different MS and MS/MS techniques are compiled in Table 1. When significant, instrumental and experimental conditions for glycero-, glycerophospho- and sphingolipids are focused on and referred to [80-88].

\section{Fatty acids, lipids and metabolites of the survey}

Fatty acids are synthetized in cells and their cell membranes, endoplasmatic reticulum, Golgi apparatus, and mitochondria [89], [90]. Most lipids are products of free fatty acids in presence of coenzyme A and NADPH [91]. This literature survey deals with a short area of lipidomics and contains commonly studied lipids, e.g. glycerolipids (GLs) like mono- (MG), di- (diacyl-, DG), and triacylsubstituted (triacyl-, TG) glycerols. [91] To look the structural challenges of 
lipids in analytics, Figure 1 illustrates the functionalities of TG lipids. Glycerophospholipids (GPs) from GLs are also included, since they are frequently detected like the most abundant lipids in eukaryotic cells, i.e. phosphatidylcholine (PC) and phosphatidylethanolamine (PE) [92]. Other GPs dealt in this review include phosphatidic acid (PA), phosphatidylserine (PS), phosphatidylinositol (PI), phosphatidylglycerols (PGs), and PG-derived cardiolipins (CLs). [92] To observe the challenges of separation and identification issues some glycerolipids and glycerophospholipids are illustrated in Figure 2.

GPs may appear with fatty acids which both are bound with ester or ether groups, the latter being defined as plasmalogens (PLs) [92]. PLs are detected and identified either by an alkenyl linker with an oxygen (e.g. PI[O-18:0/17:0]) or with a phosphorous (e.g. PI[P-18:0/17:0]) [92]. Those structural hetero atoms help mass spectrometric detection, although there may not be improvements in chromatographic separation.

An interesting group of surveyed lipids is sphingolipids (SPs, Figure 3, [28]). They have a basic backbone with various kinds of lipids. The backbone is formed from serine amino acid and a long-chain fatty acyl catalyzed by coenzyme A in mammals [92]. The analytics is focused to ceramides (Cer), which have amine-bonds with fatty acids. To sum up, interest is focused on esterified fatty acids with glycerol head groups (glycerolipids, glycerophospholipids) or a sphingosine headgroup (sphingolipids), but not a sugar backbone (saccharolipids, a topic of worth its own review). Other groups left out are defined by characteristic hydrocarbon structures, i.e. fatty acyls, prenol lipids, sterols and polyketides [94]. More analysis on lipid classes and metabolism is discussed elsewhere [101]

\section{Instrumentation}

In lipidomics, a wide range of articles introduce new or improved methods which are validated with UHPLC-MS, capillary-UHPLC-MS (nUHPLC-MS or nano-UHPLC-MS) instruments (Table 2). Most of these studies concentrate on sample preparation [14], [20], [46], [66], 
development of chromatographic methods [9], [22], [27], [45], MS/MS identification [7], [16], [23], [27], computational methods for improvement of measurement performance [17], and data analysis [21], [25], [96]. Recent challenges in HPLC-MS seem to be fluctuation (repeatability) of analysis [97], [98] results, which hinder reliable identification and interlaboratory tests of lipids. Since in chromatographic environments elution of different lipid types and their species may differ [98], deconvolution of data via manual or computational analysis may be the only reasonable compromise.

The reviewed papers show that all lipid analyses have fluctuation of lipid composition and intensity based on the fingerprint profiles of different biological matrices. These observations can be explained by distinctive matrix effects, which in turn inform that there is a specific need of sample preparation before analyses. Usually, lipidome studies are done with simplified sample preparation to avoid recovery losses in clean-up steps. [99] Mostly, the clean-up steps are protein precipitation and extraction of solid matrix compounds (e.g. biological tissues) and fluids (e.g. plasma, serum, lavages, cell suspensions and supernatants).[99] Furthermore, solid materials may trap internal standards and analytes, which increases variation of results in quantification and leads to less accuracy and precision of the methods. [100] Sample preparation of biological and clinical samples is discussed more elsewhere [101].

Though reconstitution with the most used polar acetonitrile - water mixture (60:40, v/v) and organic isopropanol - acetonitrile (90:10, v/v) eluents is practical, none of these dissolve lipids comprehensively. For instance, acetonitrile - water at 60:40 (v/v) recovers STs and TGs incompletely. [45] Furthermore, Danne-Rasche et al. [45] observed a distortion or even loss of lipids with i-propanol - acetonitrile mixture $(9: 1, v / v)$, when the eluent was used in nanoUHPLC. Thus, lipids need to be reconstituted into a standardized mixture such as butanol - ipropanol - water (8:23:69, v/v/v) [45] prior to injection. The solvent composition is important for lipid solubility. Due to high solvent volumes from pretreatment processes at the end of 
sample preparation, all lipid samples need to be dried for getting concentrates of HPLC-MS research. Furthermore, concentrates are needed to measure multidimensional MS spectra to obtain accurate identification and algorithm-based data handling of features.

To reduce systematic noise and other disadvantages (e.g. adsorption, peak broadening, adduct formation) in chromatographic data has been attempted via computational methods, i.e. with the sliding window adduct removal method (SWARM). [102] SWARM is based on the systematic interference caused by multiple adduct ions of the same analyte. The interference induces systematic noise patterns that may be excluded to enhance accurate mass acquisition for metabolites. Then, the adduct signal overlap correction for low-to-moderate resolution mass spectra could be utilized.

Modern instrumental separation and detection methodologies are still on-going needed to avoid frequent appearance of false negative lipid identifications and to establish sufficiently reliable and label-free (i.e. standard-free) methods. Identification of lipids showed to be improved by multiply usability of analytical variables and by using more independent properties, such as chromatographic retention times (RT) and mass spectrometric mass-to-charge ratios $(\mathrm{m} / \mathrm{z}$ ) of lipid ions/adducts, fragmentation/isotope patterns, and collision reactions. Reliability for targeted lipid species identified by internal standards and calibrants have made lipid analyses possible in quite many case, but especially non-targeted lipid analyses demand the super power and speed of computers with algorithm-based libraries.[96], [103], [104] Thus, the analyses can be independently conducted without potent analytes. Evidently, the computerized methods have utilized automated systems when internal standards for normalization are neglected. [2], [3], [10], [11], [13], [14], [16], [17], [20], [21], [22], [24], [26], [30], [32-36], [38], [44], [4 6], [47], [59], [63], [66], [73].

\subsection{UHPLC}


HPLC techniques are preferred for their ability to enhance identification by predictable retention time patterns [98], [105] and reduced mass ambiguity [106]. In lipid research, the sub-method UHPLC is preferred over HPLC due to its lower dispersion, substantial decrease in solvent usage, shorter analysis times without compromising resolution, higher resolution performance due to smaller (sub-2 $\mu \mathrm{m}$ ) fully porous particles or (sub-3 $\mu \mathrm{m}$ ) core-shell material [73] in columns (enabled by higher pressure capabilities) and enhanced retention precision. [107]

Our dataset [2-79], [108] informs current lipidomic UHPLC-MS separations to be primarily done with additive-assisted reversed phase liquid chromatography (RP-HPLC) hyphenated with separate experiments on positive electrospray ionization (pESI) and negative electrospray ionization (nESI). Though ESI was predominantly used for UHPLC-MS, some atmospheric pressure chemical ionization (APCI) studies were also conducted. [23], [44], [47]. ESI as a 'soft' and APCI as a 'hard' ionization technique, respectively, are suitable for supporting lipid identification. [23] In respect of that, Beccaria et al. [23] developed a method suitable for both detection approaches with no need to change HPLC parameters.

Elution of lipids in HPLC is done in many elution models (e.g. isocratic elution, curved gradient following an exponential or logarithmic function, stepped gradient, linear gradient, etc.). The suitable stationary and mobile phases with methods are also found in Table 1. More detailed information on stationary and mobile phases as well as lipid adduct concentrations is in Supplementary (Table S1).

\subsubsection{Reversed phase separation in HPLC}

In lipidomics the commonly used RP-HPLC separation methods are based on the interaction of a nonpolar stationary phase with nonpolar lipid analytes. Nonlinear or second-degree curves between the retention times and total carbon $(\mathrm{CN})$ double bonds $(\mathrm{DB})$ are used for quantitative calculations. [9], [16], [98], [105] For example, they help to differentiate possible sodium 
adducts from similar exact masses (e.g. $[\mathrm{PC}(38: 4)+\mathrm{Na}]^{+}$and $\left.[\mathrm{PC}(40: 7)+\mathrm{H}]^{+}[9]\right)$. However, retention time prediction can be complicated due to $\mathrm{pH}$ dependent speciation [109], experimentally made validation and multi-step liquid gradients [23]. Separation of polar components (i.e. early retaining lipids) can be affected by the mobile phase gradient. When elution is started with high percentage of organic mixture, polar compounds are longer adsorbed and retained on RP-HPLC phases. [110] However, changes of eluent polarity are interlinked with the ionization rate of polar lipids, which in turn can have an impact on elution. [110] RP-HPLC with polar solvents (i.e. non-aqueous RP-HPLC) is enabled by polymer-based columns which are end-capped by a methyl or hydrogen group. [110] Some column materials are based on modified silica, which when not shielded are short-periodically used with water modified organic solvents due to free silanol groups having the attractive functions. [110] In contrast to phenomena in reversed phases, normal phases in HPLC (NP-HPLC) typically separates analytes containing polar functionalities having silanol, amino- or diol groups. [111] NP-HPLC is excluded from the review, since only a single article on NP-HPLC with amide column was observed during 2017-2019 on lipid separation [31].

Since even the variation among lipid class species can be substantial, it is not surprising that the polarity of their classes varies a lot. Tumanov and Kamphorst [85] (Figure 4) demonstrated the lipid-subclass range of four distinct chromatographic approaches, which are divided to two groups (one a RP, one a NP separation strategy in each group). The subclasses in (A) include lipids of the nonpolar kind, whereas the separations of (B) are modelled to the polar lipid subclasses. Particularly, polar (lyso-forms, MGs, sphingosine-related compounds, FAs, acyl carnitines and acetyl coenzyme A) and mid-polar (PS, PG, PI, Cer) lipids seem to be species often analysed with the negative ionization mode, though more species are primarily found with positive ionization. From the GLs only MGs have FA chains with 16 counts, i.e. they are small enough to be rather polar. As a thumb rule, SPs are on the mid-polar lipid spectrum, when 
they have amide-bound FA chains. [85] Thus for reliable research data, accurate UHPLC-MS methods are needed to identify the individual lipid class species.

\subsubsection{HILIC}

Essentially, hydrophilic interaction liquid chromatography (HILIC) columns are micro-bore columns with stationary phases of mixed hydrophilic interactions for nonpolar compounds and hydrophobic interactions for polar compounds. However, nonpolar lipids (e.g. CEs, TGs) and lipids with only one hydroxyl group (e.g. Cer, DG, MG, and cholesterol) are often barely retained. [16] Nevertheless, HILIC is a well-established subclass of NP chromatography that allows usage of water as the eluent $(5-40 \%,>2 \%$ needed) as in contrast to conventional NP. [112] HILIC platforms are ideal for quantitation, since lipid class species co-elute with their respective calibrants. [109]

PAs and PSs species are known to have broad or barely detectable peaks in RP-HPLC, thus, HILIC is used. [112] Furthermore, as PAs and LPAs co-elute among major lipid components (e.g. PCs, PEs, SMs) in RP-HPLC. Because of that the comparatively lower abundance of lipids in this class, PAs and LPAs have no selective fragment to differentiate from other lipid classes. Thus, proper methods for PA/LPA separation have been specifically approached with HILIC. [113], [109]

Though being a well-established technique for HPLC-MS quantitation, not many UHPLC analyses have used HILIC-columns for separation. [16], [47] Thus, HILIC applications are also excluded from the scope of this review.

\subsubsection{Nano-UHPLC}

In nano-HPLC [112] columns of sub-millimetre internal diameter (i.d.) are filled with conventional column packing materials. Published papers [41], [71], [24], [45], [42] inform only about using a loaded capillary (i.d.: 1-0.001 mm [114]) or a nanobore column (i.d.: 0.1$0.025 \mathrm{~mm}[114])$. For comparison, a large majority of the lipid studies are done with the 
smallest possible narrow bore columns $(2.1-4 \mathrm{~mm})$. Surprisingly, along with nano-HPLC a low-resolution triple-quadrupole mass spectrometer was unequivocally preferred.

In contrast to a faster UHPLC separation, in nano-HPLC the "loading time" followed by the sample elution was extended due to the low flow rate restrictions $(0.3-0.35 \mu 1 / \mathrm{min})$. [71]

Nano-HPLC methods show a great capacity for lipid identification with a low-resolution mass analyser, since then both an extremely broad identification range and high sensitivity at the low fmol-scale are gained using a low throughput (analysis time: 40-60 min). [41], [45] The result was that the low fmol range for almost every GLs, GPs, SPs and their lipid derivatives was reached in the positive ionization electrospray mode (pESI). For example, the calibration curve for a specific PE (17:0/14:1) demonstrated a linear relationship between 16-10000 fmol by pESI-MS detection and 0.64-2000 fmol by nESI-MS detection [41] By contrast, with HPLCESI-MS the linear dynamic range for the same analyte reached around 80-10000 fmol by pESIMS and 16-2000 fmol by nESI-MS. [45] Similarly, Kim et al. [115] achieved a LOD-range from 59 fmol (LPC(17:0)) to 507 fmol (LPG(14:0)) with untargeted nano-UHPLC-ESIMS/MS of lipoprotein by pESI and nESI ionization, respectively. [115]

\subsubsection{Mobile phases in hyphenation of HPLC with MS}

Mobile phases in HPLC are usually modified to help in lipid detection and their separation with HPLC. [112] As the sensitivity issues are concerned, composition of eluents is important to obtain stabile adduct ions in MS. [23] Volatile buffers (e.g. formic acid, acetic acid, or their ammonium salts [116]) are used in lipidomics methods. [113] However, earlier studies report lipid results with $5 \mu \mathrm{M}$ phosphate buffer (nanoelectrospray ionization) [9] and $5 \mathrm{mM}$ phosphoric acid [45]

In some cases, additives in HPLC eluents may cause analytes to become undetectable due to signal suppression. Cajka and Fiehn [117] studied the effects of five different modifiers in both pESI and nESI mode with two different HPLC columns filled with ethylene bridged hybrid 
(BEH) particles and the slightly better charged surface hybrid (CSH) particles. [117] Whereas the experiments could be concluded by choosing the optimal mobile phase modifier to be formic acid or formic acid/ammonium acetate for $\mathrm{pESI}$ and ammonium acetate for nESI. Using a CSH C18 column with HPLC-pESI-MS studies Monnin et al. [118] reported about a further enhanced ionization efficiency by choosing $0.02 \%$ acetic acid as the additive in eluent. Whereas the signal for LPLs (except LSM) and GPs certainly increased by manifold, Cer and PCs experienced a decrease in peak area when compared with analyses data with $10 \mathrm{mM}$ ammonium acetate. [118] However, specific to PCs their carbonate ion adducts showed 20fold stronger signals compared to their ammonium adducts with CID-MS/MS. [119]

\subsection{Importance of mass spectrometry detection in lipid research}

Formation of adducts is dependent on the molecular structure and functional groups of a lipid (e.g. deprotonation of carboxyl groups in fatty acid with nESI). Lists of exact masses in MS and MS/MS, non-protonated adducts, possible collision energies and lipid classes/topics are listed in Table 3. Furthermore, sources like Lipid maps providing a free MS/MS prediction tool (http://lipidmaps.org/resources/tools/index.php) and a structure database library (http://lipidmaps.org/data/classification/LM_classification_exp.php) allow data handling in lipidomics.

MS advancements, such as a quadrupole Orbitrap mass analyzer (Q-Orbitrap) [9], [17], [20], [22], [27], [31], [46], [49], [51], [55], [56], [62-65], [77], [79], and NSI [24], [45], [71] have extremely increased resolution capabilities (theoretical plate number in HPLC >35 $000 / \mathrm{m}$ ) for lipid identification. However, compromises need to be done before the analyses, since either mass accuracy or ion resolution is emphasized depending on whether identification or quantitation is preferred. Furthermore, identification is improved with the help of orthogonal measurements, such as collisional cross-section (CCS) values with ion mobility spectrometry (IMS), achieved with a drift tube coupled with MS instruments, such as a quadrupole time of 
flight (QTOF) [6], [16], [25], [68]. Furthermore, the inclusion of trapped IMS (TIMS) combined with parallel accumulation serial fragmentation (PASEF) has emerged to enhance complementary MS/MS and IMS data. [126]

For high-resolution MS, an Orbitrap [2], [5], [34], QTOF [4], [6], [10-16], [21], [25], [28], [30], [32], [33], [36-40], [43], [44], [53], [54], [59-61], [66], [68-71], [71], AA073, [74], [75], AA076, [78] and QOrbitrap [3], [5], [9], [17-20], [22], [26], [27], [31], [34], [35], [46], [47], [49-51], [55-58], [60], [62-65], [72], [77], [79] were used. However, some studies settled for low-resolution MS, mostly with a triple quadrupole instrument [7], [8], [23], [24], [30], [4143]. Most rarely, pseudotargeted methods [21], [30], [54] (i.e. lipid identification with an in silico library), polarity switching [5], [17], [23], [41], [49], standalone nESI mode in MS [29], [43], [68], NSI [45], [71] and atmospheric pressure chemical ionization (APCI) [23], [44], [47] were used.

\subsection{Data acquisition and processing}

Identification and determination of lipids and their metabolites need commercial, open-source, and sophisticated tailor-made tools [127]. The statistical tools, analysis software, and algorithms enable visualization and perceiving of patterns from large datasets and raw data [103]. Metabolic profiling of MS data is done with multi-variant programs, such as Metaboanalyst, [10], [11], [17], [27], [32], [34], [37], [40], [46], [59], [63], [77] or MeV [3], [30], [65], [61], [79], [103],. All in all, reported software environments for data processing constitute mostly of R and SPSS languages.

Manual programming from the ground up or with borrowed code demands more computational expertise. Koelmel et al. [96] compared identification algorithms with the commercial R-based LipidMatch-tool, which is tailored for lipid identification softwares. [96] For a deeper understanding of differences in program functionalities, the article on "LipidMatch: an 
automated workflow for rule-based lipid identification using untargeted high-resolution tandem mass spectrometry data“" gives excellent information [96].

\section{Analysis}

\subsection{Identification of lipids}

Lipid identification can be enhanced by using information about the individual lipid itself, e.g. by increasing orthogonal information via "polarity switching" [5], [17], [23], [41], [49] instead of only using one ionization mode in the measurements or by the introduction of supporting measurements or (lipid class) expanding methods. Identification demands are partly already mentioned in Introduction and chapters, where chromatographic parameters are discussed referenced to resolution and sensitivity.

In lipid studies, absolute retention time (RT) variation during sample analyses is easily increased by small differences in experimental conditions (preparatory, chromatographic, and instrumental parameters). However, more repeatable separation techniques along with feature alignment enables more reliable identification with RTs in inter-laboratory studies in the future. [128] Since measurements of $\mathrm{m} / \mathrm{z}$ ratio are significantly less deviant (parameters affecting $\mathrm{m} / \mathrm{z}$ : ionization efficiency and mass spectrometric setup), single $\mathrm{m} / \mathrm{z}$, feature, and low-resolution spectra matching are the most used means for quick identification. [7] If needed, high resolution precursor and product ion spectra are usually obtained to achieve accurate identification between highly similar species. For further optimization of mass spectrometric identification, adduct formation and control of collision energy should also be studied and used. It is commonly agreed that adduct ions are formed mostly during ionization [9], [73], which is why lipid species appear at the same retention time in both pESI-MS and nESI-MS. Apart from protonated and deprotonated species, adding of millimolar concentrations of salts can be harnessed for signal enhancement [129]. Though at too high concentrations lipid signals can drastically be inhibited due to background noise caused by ion clusters [129]. Common ligand 
ions are acetate $\left(\mathrm{CH}_{3} \mathrm{COO}^{-}\right)$[56], [68], formate $\left(\mathrm{HCOO}^{-}\right)$[48], [50], [57], [58], [60], [75-77], and ammonium $\left(\mathrm{NH}_{4}^{+}\right)$[48], [50], [56-58], [60], [74-76]. Sodium (Na+) and potassium (K+) adducts are also commonly observed, though no controlled addition of such salts was noticed [92].

High resolution RP-HPLC separates structural isomers of lipid classes, [9] up to the point of carbon chain regioisomers (i.e. sn-positions of lipids). However, comprehensive identification of isobars and isomers could not be achieved by solely using MS/MS. For example, Lisa et al. [16] and Blaženović et al. [25] reported unresolved isomers and isobars with identical CNs and DBs but different fatty acyl composition. Adducts with same nominal mass can be separated with MS/MS [23], but specific information like sn-position of DBs needs other methods, like derivatizing of free fatty acids to form 4-iodobenzyl esters [130]. The reaction is made to esterified fatty acids into distinguishable epoxides via ketone dioxide catalyst and oxidant [131] or to oxetane-adducts via the Paternó-Büchi photochemical reaction. [132], [133], [134] Mass spectrometric solutions include second-generation fragmentation (MS/MS/MS) by dual stage collision-induced dissociation (CID) [135] or CID and ozone-induced dissociation combined, or silver-ion chromatography (currently achieved only with TGs) [81], [112], [136]. Recently, Zhao et al. [119] also mentioned electron impact excitation of ions from organics (EIEIO) and photo-ultraviolet dissociation (PUVD, photodissociation [130]) in the list of nonCID methods for sn and DB position. Since lyso-forms of GPs, i.e. sn-2 acyl lipids retain less in a HPLC column than their sn-1 isomers, they can be identified as separated double peaks. [9]

\subsection{Quantification of lipids}

Burla et al. [99] (a statement of the global lipidome community) compiled guideline recommendations for quantitative analysis in clinical sample matrices, specifically absolute quantification of lipids in plasma and serum. For the quick comparison of absolute 
quantification results, lipid concentrations should be reported in the SI-units mol/L or molar percentages whenever possible. [99]

In comparison to lipid identification, quantitative analysis is increasingly dependent on control samples as well as the quality and concentration of included standards, i.e. internal standards and calibrants. Common control samples include blanks and quality control (QC): Blanks are included for standard impurity monitoring and validation purposes, whereas QC samples (a pooled sample of all study samples in a batch) serves to monitor and correct batch-related uncertainty, instrumental errors and evaluate the performance of lipid analytes for validation purposes. [99]

Absolute quantitative calibration of lipids is either conducted via spiked QC samples (surrogate calibration) or by direct introduction of standards in the study sample as is often done in singlepoint and standard addition calibration. [75] However, single-point calibrations are reported to overestimate target lipid concentrations due to inherent flaws in the regression. [75] An ideal calibrant for complex matrices has an identical response factor, ionization efficiency and experiences comparable matrix effects as the analyte. [137]

For better understanding quantitative UHPLC-MS, some differences between HPLC-MS and DIMS should be pointed out. Though DIMS is more robust at the high-concentration lipids $(\mu \mathrm{mol} / \mathrm{L}-\mathrm{mmol} / \mathrm{L})$ of a sample, HPLC-MS is advantageous for the identification and quantification of low- and very low-abundance lipids (<nmol/L-mmol/L). [137], [99] Anyway, lipid class coverage and sensitivity are considerably improved in HPLC-MS experiments. [99] As an inherent disadvantage in HPLC-MS, the risk of lipid-lipid interaction increases due to the enrichment of same lipid species occurring in the column, though hetero-interaction between different lipid species decreases. [137] HPLC-MS uses peak areas in contrast to DIMS, where more stable ionization conditions enable quantification via peak intensity. [82] 
Moreover, the fluctuating quality and quantity of molecular species in the ion source is a recurring problem for lipid quantifications. These issues are amplified by the increasing complexity of experimental setups, such as chromatographic gradients affecting electrical properties (i.e. ionization efficiency) of the eluent. This has implications not only for the ionization efficiency, but also for the MS instrument's response factor: alongside solvent composition, Drotleff et al. [75] reports post-acquisition $\mathrm{MS}^{\mathrm{n}}$ procedures like SWATH or multiple reaction monitoring (MRM) to further deviate the detector's response values from one unit per molecule. Drotleff et al. [75] proposed "post-acquisition recalibration" as a reasonable compromise, if not enough sample is available for using the standard addition method. Then, sensitivities of analytes can be determined via a certified reference material. For lipids, only the NIST CRM 1950 plasma reference is currently acknowledged. [75] Standard mixtures, such as the Splash ${ }^{\circledR}$ Lipidomix $^{\circledR}$ mixture (Avanti Polar Lipids Inc., Alabaster, AL, USA) have been used for mimicking corresponding lipid concentrations in experiments as in biological fluids, e.g. human plasma, and for normalization of the analyses [14], [28], [66]. Since lipidome isotope labelling of yeast (LILY, complete lipidome carbon labelling with C13 isotope [138]) technology is discovered very recently, studies on using it in practice could not be considered in this review.

Furthermore, solvent-system dependent lipid concentrations of $>10-100 \mathrm{mmol} / \mathrm{L}$ are reported to form significant amounts of poorly ionic lipid aggregates (results found in a DIMS experiment). [137] The formation of aggregates like dimer, oligomer and micellar structures is further increased by the hydrophobicity of the lipid analyte and polarity of the mobile phase. [137]

Biological matrices include inherent variation in concentrations. UHPLC-MS experiments mostly revolve relative quantification and study changes in the lipidome of biological systems. However, in general HPLC-MS lipidomics has grown enough to advance further in absolute 
quantification of targeted lipids. [99] Main limitations of absolute quantification in HPLC-MS concern ambiguities preceding calibration, i.e. the structural versatility of lipid species, lipid concentrations at the lower (or upper) border of their linearity range, and various matrix effects such as ion suppression and enhancement. [99] Whereas polar lipid species quantification reaches a $\sim 5 \%$ accuracy (due to the polar headgroup predominantly explains their MS sensitivity) [137]. Mid- to nonpolar lipids like TGs and cholesteric esters are more effected by their specific structure in ESI-MS. [104] Thus, these lipids need more attention in standard representation. More details on quantitative and validation in lipid analyses are informed in Refs. [99] and [104].

\section{Data analysis of lipids}

According to the lipidome community, experimentally acquired raw data should be available for result-validation purposes and study re-analysis, whereas analytical details and results should be included in a file with acknowledged "XML[-based] or structured tabular format". [99] Corresponding raw data information can be taken from the proteomic field, which has practical formats for efficient data sharing. [99]

\subsection{Normalization}

Normalization of raw data is used to reduce systematic fluctuation concerning an accurate measurement. For example, Boysen et al. [139] developed the best matched internal standard normalization (B-MIS), an algorithm-driven solution for correcting non-biological variation of raw data (i.e. ion suppression by matrix effects, chromatographic quality and analytical drift). Obscuring variation, i.e. changes of peak area as a function of concentration, is compared between an unrestricted set of internal standards and analyte peaks in a QC sample. As a result, the algorithm chooses a suitable standard for normalization if the relative standard deviation of the peak area is improved by 40\%. [139] Furthermore, Drotleff et al. [76] considered B-MIS to be the best normalization model for reducing intragroup variation between the coefficient of 
variation, median absolute deviation and variance. His team observed a small difference in real positives favouring this normalization, but also noted that normalization models may work in a complementary fashion with their respective strengths and weaknesses. [76] Though not yet applied in absolute quantification experiments, B-MIS appears as a good alternative for modelbased peak area normalization in relative quantification methods. [139], [76]

Normalization is done with single standards representing the whole range of lipid classes. Suitable internal calibration standards with the same head groups and similar, but not identical fatty acids (e.g. odd-numbered $<1 \%$ abundant in higher organisms [104]), or isotope-labeled fatty acids are preferred for normalization [104]. The most used internal standards are deuterated at the carbon chain of their FA end to have either 7 or 9 deuterium atoms. [75] Internal standards included in QC samples are used for post-correction of systematic errors (i.e. drift and other batch-biases) [104], [99] like changes in peak shapes influencing the peak areas and for monitoring carry over [112].

\subsection{Data libraries}

Typically, lipid metabolite features in untargeted analyses are compared to available library spectra. [66], [118] Therefore, acquired data is represented by a "closest-match" for the $\mathrm{m} / \mathrm{z}$ or total ion spectrum that is compared, typically accompanied by a score from 1-100\%. Evidently, this approach may lead to false positive results, when the database used is incomplete or when the reference data is noisy [128].

Along with home-made databases [30], [32], [33], [36], [51], [54], [68], [78], commercial and open-source libraries have gained popularity and variety both for monitoring the lipid range with MS by characterizing fragmentation patterns for selective identity searching of lipids. Information about individual libraries and their respective types are compiled in Table 4. Specific tools like open-source softwares are mentioned in Table 5, whereas chemometric and statistical concepts are introduced in the next chapters. Additional information can be found 
from the LSI document (https://lipidomics-standards-initiative.org/links) and LIPID MAPS pages (http://lipidmaps.org/resources/tools/index.php).

\subsection{Chemometric tools}

Typically, metabolic correlation and significance analyses of lipids is conducted with a set of visual chemometric analyses and statistical methods. Chemometric methods provide an overview on analyte trends and outliers in relationship to their observations (features, spectra, etc.) with principal component analysis (PCA) [5], [7], [13], [16], [17-19], [27], [30], [31], [3336], [38-40], [43], [47-49], [51], [52], [54], [56-60], [62-64], [66], [68], [69], [72], [73], [7579], [124], systematic but hidden/uncorrelated variation between lipidome states with partial least-squares regression - discriminant analysis (PLS-DA) [3], [7], [10], [11], [26], [32], [34], [35], [48], [59], [60], [64], [76], [79], or orthogonal projection of latent structures discriminant analysis (OPLS-DA) [4], [6], [9], [19], [27], [33], [36], [37], [49], [50], [52], [54], [57], [60], [69], [78], [79], (relative or exact) concentration evaluation with boxplots [10], [18], [19], [32], [34], [37], [40], [44], [45], [48], [58], [60], [61], [67], [69], [73], [76] or a heatmap [3], [5], [7], [10], [11], [17], [26-28], [30-32], [34-38], [40-42], [45], [47], [48], [52], [56-58], [62], [68], [69], [73], [77], [79] visualizations for significant outlier detection such as the Bland-Altman plot [16], [40], [43-45], [55], analyte interaction or metabolic interlinkage of lipid species via hierarchical cluster analysis (HCA, often included with heatmap analysis) [3], [5], [7], [10], [17], [26], [27], [34], [37], [39], [40], [47-49], [68], [69], [73], [77], [79], interactive network [3], [26], [47] or pathway analysis [3], [7], [11], [26], [27], [37], [60], and diagnostic tools such as importance testing of PLS-DA variables with variable importance projection (VIP) [3], [10], [26], [33-37], [48], [50], [52], [57], [64], [79], OPLS-DA variable reliability and importance evaluation via S-plots [6], [27], [50], [57], [69], [79] or volcano plots [11], [31], [41], [42], [48], [60], [63], [67], [74], [77], [78], and method or sample comparisons with Venn diagrams [11], [17], [27], [30], [45], [49], [60], [66], [76]. 
PCA [140] pursues to flatten a large dataset with multiple variables to find the most important two sources of variation, i.e. principal components. This is done by comparing the transitions between all variables and flattening them into two dimensions with matrix operations. Thus, the most important information about multivariate data is found. This reduction process may help to divide the studied compounds into different groups and characterize them. [141] Similarly, PLS-DA seeks to flatten multivariate data to find the most fitting parallel twodimensional plane representing the whole dataset, [140] whereas OPLS-DA reduces the dataset to an orthogonal plane found with the partial least square plateau of the dataset. [142] Briefly, HCA clusters and categorizes sample components according to their apparent covariant relationship. [143]

Before actual multivariate data-analysis, the data needs often to be transformed, i.e. cleaned, scaled and re-centered, as is often done with e.g. statistical noise corrections or discarding of noisy data/spectra/outliers, unit variance or Pareto scaling and mean centering for PCA. [144] Chemometric methods, such as PCA, PLS-DA and OPLS-DA have kept their positions as the most common tools for visualizing analyte groupings (PCA) and metabolic changes (PLS-DA, OPLS-DA). Furthermore, VIP has emerged as an emphasis estimator of PLS-DA variables [3], [8], [26], [32], [34], [37], [64], [79] emphasizing the importance (i.e. magnitude) of each variable. In this context, lipids in the projection plot with VIP scores $>1$ are most accountable to predict changes in metabolism. More extensive analyses can be found in Ref. [88]

\subsection{Statistical tools}

In lipidomic research, statistical methods have developed into a broad variety of numerical tests and visualization techniques. Targeted and untargeted methods are two distinctively different approaches, as well as the means of data processing. Gorrochategui et al. [103] divided the targeted processing of metabolites into five phases: (1) the acquisition of raw data, (2) the contemplation of which database should be used when considering the research question and 
analytes, (3) the pre-processing and identification of these metabolites, (4) the normalization and quantification of the identified species, and (5) the reflection of the results on the biochemical/physiological context of metabolic pathways. [103] In contrast to targeted approaches, untargeted approaches need more careful experimental MS data processing, data modification, and evaluation for pre-processing to attain sufficient data quality. By this way, relevant biomarkers can be identified from the totality of detected features/peaks. The data quality is directly affected by the sensitivity and resolving power of the applied instrumentation. The non-targeted steps include the same pathway elucidation as in the targeted analyses, however, with respective appropriate tools. [103]

Statistical methods include a null hypothesis to test similarities by using one dataset. However, the $p$-value parameter in the Student's $t$-test [2], [10], [14], [15], [18], [19], [26], [27], [32], [37-39], [42], [50], [52], [54], [56], [57], [60], [61], [63], [67], [72], [79], the non-parametric Mann-Whitney $U$-test, [15], [31], [35], [41], [50], [57], [58], [74], [77], and the analysis of variance test (ANOVA) [2], [7], [10], [17], [22], [27], [32], [33], [46], [64], [69], [79] are for comparison of two or more datasets. These methods are often used for validation of analytical data and for detection of changes (e.g. analyte concentrations) between a control sample and authentic samples. Together with statistical tests, correction methods such as the BenjaminiHochberg test [145] are used to calculate the false discovery rate (FDR), i.e. minimizing false positive data in the dataset [56-58], [60], [74], [76].

The $t$-test designed for comparing two independent variables (e.g. patient versus control group lipidome data and that before and after drug intake) may be unreliable, when the sample size number is small $(\mathrm{n}<30)$ or when other kinds than normal distributions arise through the data processing [63]. This is the case for most sample number described in the articles reviewed for this paper. The average number of real samples studied was around 10-20 with the excluded outliers of 283 [36] and 10115 [94] test subjects. As to analyse control samples, there was a 
clear split of 7-16 and 30 in the number of controls used in the individual studies [30], [34]. Some cases of statistical testing of the datasets may be questionable, since e.g. $t$-tests were not reliable, when variances between populations were different. [146] For these kinds of cases, an unequal variance test like the Storey [74], [115] or Welch [48] $t$-test may be used instead [48], [74].

The Mann-Whitney U-test [147] (also known as the Wilcoxon rank-sum test) is used in mathematical data handling, when there are not any fixed parameters, which can be used to evaluate similarities of two independent dataset medians with a null hypothesis. For two sets with same or different sample sizes, the values of both datasets are sorted from the smallest to the largest one. The ranking integer is divided, if two values are identical: for instance, when two datapoints have the value 2 , they will be both noted as 1.5 [147]. Then, the separately ranked sample value sets are individually summed. When any of the sums reaches the critical value range of the $U$-test, the null hypothesis can be rejected. Akin to the Student's $t$-test [63], the $U$-test is evaluated by determining the $\mathrm{z}$-score $\left(z=\frac{x_{i}-\mu}{S D}\right.$, where $\mathrm{x}_{\mathrm{i}}$ is a value of a single datapoint, $\mu$ the population mean and SD the population standard deviation) from a normal distribution, suitable for a large number of studied samples (>30) [147]. In essence, the z-score is a distance-measurement of a single datapoint in relation to a normal distribution's standard deviation, determining the distance between its mean and single measurement.

A Mann-Whitney $U$-test [147] can be fitted for non-Gaussian distribution data. It is typically combined with a Benjamini-Hochberg (or Bonferroni-Holm [63]) test to exclude false positive values, thus providing the FDR. These methods were applied in multiple studies to limit uncertainty in the results of lipids. Furthermore, Paepe et al. [27] and Gong et al. [60] used cross-validated ANOVA test (CV-ANOVA) to improve reliability of the identified analytes. The reviewed papers also gave information that Mann-Whitney $U$-tests and FDR corrections 
with the Benjamini-Hochberg test [145] were used to discover the real positive results from analysed MS datasets.

In the Benjamini-Hochberg method [145] p-values of two sample sets (between the control and the test groups) are inflated mathematically to reveal possible false positive results. Since affected and unaffected lipids represent two distinct normal distributions, false positive values can be ideally seen to differentiate a distribution from the normal when using a big sample number in the study. In the data analysis of lipid species, the original $p$-values are inflated and excluded by their significance desired for the $p$-values (e.g. $p=0.1,10 \%$ significance). The $p$ values are then ranked from the smallest to the largest, after which the individual $p$-values are converted to a largest-to-smallest sequence. The largest $p$-value is kept, but the second largest $p$-value is determined as the smaller value of the two options. Therefore, either it is the same value preceding it, or it is the value calculated with a separate equation [145].

Lately, Tietz-Bogert et al. [31] calculated the FDR value in a lipido-metabolomic study by searching significant biomarkers of primary sclerosing cholangitis [31], which is a disorder of lipids in the bile duct of unknown origin. The concentrations of lipid species in control samples of healthy individual's and the clinical samples of sick patient's blood and bile were compared to find changes with a statistical significance of $p=0.01$ [31]. Simpler classical methods like ANOVA for multiple variables [2], [32], [46] were only occasionally used.

Machine learning and software advancements for multi-ionic identification have allowed to evaluate MS spectra produced with UHPLC-MS in silico, which is only demonstrated in a few recent articles within our scope [3], [18], [21], [25], [33], [67]. Due to machine learning techniques and their discovered use in omics, automated lipidomic analyses, receiver operating characteristic curve/area under curve (ROC/AUC) cross-validation analyses [3], [60], [67], [76] random forest studies [18], [32], [59], [67], neural network applications [67], [96], in silico spectra evaluation [21], and CCS value generation algorithms [25] have become in use. 


\section{Discussion}

UHPLC-MS lipidomics is divided into isotope-labelled standard methods and fully standardless algorithm-based analyses. Furthermore, the separation and acquisition of highly abundant lipid classes $(\mathrm{mmol} / \mathrm{L})$ from mid- $(\mu \mathrm{mol} / \mathrm{L})$ and low-abundance $(\leq \mathrm{nmol} / \mathrm{L})$ classes and their species is a challenge. [113] This is especially the case when (semi-)quantitative analysis is conducted for all quantifiable lipids in the sample, which can be the case for indepth studies on biological materials.

When comparing matrix effects in pharmacokinetic analysis made with HPLC and supercritical fluid chromatography (SFC) coupled with ESI-MS/MS, Svan et al. [148] observed a higher amount of ion suppression in SFC than in HPLC due to more ion enhancement. Is this general notion also applicable to HPLC-MS in lipidomics? Further information may be obtained from matrix effects via post-column infusion [148] as demonstrated by Drotleff et al. [75] or observations from more laborious (though more informative) post-extraction process. [149] Both of these tools may be important for improving the understanding of lipidome analysis in the future, as sightings of such matrix effect analysis is rare in recent lipid papers.

For practical reasons, a limitation of the comprehensive lipidome analysis is caused by the lipids without reasonable sensitivity. [9] Thus, the solution in that case is the Pareto principle, i.e. a limited number of biomolecules (lipid species) can be explained and measured using the main part of the studied biomass. [9] This is especially understandable in studies, where all the components are not relevant to the scientific question proposed. For example, metabolic lipid profiling with uncorrelated variation analysis (PLS-DA, OPLS-DA) in combination with metabolic knowledge highlight certain lipids from others to determine the most viable biomarkers. These biomarkers may form a conjoint array, which can already explain the changes in metabolism. 
The studies reviewed revealed a constant change, which affect retention time and peak focusing in a HPLC method: flow rate, gradient and oven temperature. Apart from an isocratic [43] and three-solvent eluents [10], [32], using aqueous - organic solvent gradients by increasing organic solvent percentage appeared in stepped (two [21], [40], [48] to three [25], [29], [38], [39] plateau steps or two [6], [69] to three [51] inclined steps), curved (exponential, [8], [37], [45], [67] logarithmic, [2], [19], [24], [41], [42], [50], [71] or s-curve [20], [27], [39], [49], [59], [63], [66]) and most popularly in linear [3], [9], [12], [13], [16], [17], [18], [22], [26], [28], [33], [34], [47], [52-54], [56], [67], [78] form. Modifications and combinations such as an inverted (i.e. increase in polar solvent percentage) linear [60], [79] or logarithmic [11] gradient, pyramidic [57], [77], pit-like [15], modified $S$-curve [5], [7], [45], [64] linear/isocratic [19], [23], exponential/linear [62], [68], [75], exponential/exponential [65], S-curve/exponential [64] and $S$-curve/isocratic [5] gradient were also used.

In particular, it was observed that the lipid studies were done under various column heating temperatures, from room temperature to $65^{\circ} \mathrm{C}$. [65] Instrumentation for keeping the oven temperature stabile is very important in lipids analysis standardization. Furthermore, the research was done under various mobile phase temperatures, i.e. lipid analytes experienced significant temperature changes (up to $10-20{ }^{\circ} \mathrm{C}$ ) from column inlet to outlet. [107], [150] In addition, as heat is propagated from the column walls to the centre, temperature gradients are evident. [107] Though this is partly considered by narrow bore packed columns and the preequilibration of the analyses, the eluents flown from solvent units in room-temperature may cool down the column unevenly resulting in separation of lipids with low resolution.

The choose of column properties in lipid analyses should be considered by sorbent chemistry [109], particle size, pore size [86], and particle technology [117]. The unanimously preferred column (i.d. $2.1 \mathrm{~mm}$ ) is not necessarily optimal, since narrower columns are more prone to wall effects. [107], [144] This is due to a relatively more heterogeneous packing in comparison to 
bigger columns (internal diameter larger, i.d.). Moreover, a bigger i.d. (3 mm) is argued to enhance column efficiency and allow higher flow rates. [107] However, this may be an insignificant issue compared to the current disparity in lipidome method standardization and validation. [97]

Avanti Polar Lipids (https://avantilipids.com/tech-support/physical-properties/ionizationconstants) provides pKa-values of phospholipids to be closest to buffer pKa at 2.6 (PS phosphate group), 3 (PAs), 2.9-3.5 (PGs) and 5.5 (carboxyl group of PS) when compared to the pKa values of formic acid (3.74 [151]) and acetic acid (4.75 [151]). This means that when those organic acids are used PSs (and PAs/PGs at low $\mathrm{pH}$ ) are never once charged ions. Further problems may arise for amines such as in sphingosine (pKa 9.1 [152]), PE (9.6 [153]) or PS (9.8 [153]) when ammonia (pKa 9.25 [151]) is used. Modifiers used as eluent additives may also lead to fluctuations of $\mathrm{pH}$ in non-buffered systems in on-line coupled HPLC-MS. [154] The phenomena are possible either in the eluent during electrochemical ionization due to charge-balancing redox reactions, and during droplet shrinkage, since it may cause structural perturbations and discrepancy in the ratios of ionization species. [154]

In lipidome analytics, interdisciplinary analyses on multifunctional and computational methods will be crucial to effective and improved data processing. Furthermore, it is important to use the most practical parameters such as precursors, fragments, and CCS values in evaluation of data to get fast identification and determination of lipids. Multifunctional methods used are statistic and chemometric analyses, whereas computational methods include algorithms, dataprocessing interfaces, specific software, and machine learning strategies. Both method categories were attempted to be refined with tailored software platforms for necessary data preprocessing, pattern recognition, and for using large datasets with an immense amount of dataprocessing interfaces. 
Concerning common terminology, Aristizabal et al. [155] suggest names depending on the acquired structural information: brutto (e.g. PC 36:1), medio (e.g. PC(18:0/18:1), sn-position not known), genio (e.g. PC(18:0/18:1), sn-position known) and infinio (e.g. PC(18:0/18:1-9Z), sn-position not known). The same research group identified two distinct trends in lipidomics, which are the global analysis of lipids (coined macrolipidomics) and the specialized analyses for low-abundant lipids (microlipidomics).

Finally, the recognition correlation of $\mathrm{HCA}$ analyses is derived from information often visualized in the Heatmaps. Since HCA sorts out (i.e. clusters) groups of similar analyte species with each other, similar tools can potentially be developed by means of identifying and distinguishing matrices from each other.

As the scope of this review focuses mainly on UHPLC applications, it may limit some aspects of progress in HPLC-MS lipidomics as a whole. However, this was deemed necessary as publications on lipidomics have drastically increased. [156], [157] In our opinion -as may be representative for lipid studies in general- the most neglected topic mainly left out in the dataset was nutritional lipidomics [133] (mainly personalized nutrition [158] and nutritional intervention [159] studies). This is in tune with the main tendency of lipidomics towards lifesciences and bioanalytical questions concerning physiology and pathology. Furthermore, UHPLC-IMS-MS $^{\mathrm{n}}$ studies were rarer than might be expected from current trends in HPLCIMS-MS ${ }^{\mathrm{n}}$ lipidomics. Our dataset between the years 2017-2019 (topics discussed elsewhere [101]) included research on lipid method development, physiological profiling and metabolic changes via multicomponent analysis including drug development and biomarker studies. Sample matrices included plants, microbiota, mammals, fish and human patients. [101] As UHPLC instruments are less frequent in the total scheme of lipidomics compared to the commonly used HPLC instruments, limitations concerning this review's scope on lipidome applications must be noted. For instance, the use of acetone in an eluent mixture was only used 
by Castro-Gómez et al. [15] though some studies use a HPLC-MS ternary gradient separation in the fashion of Graeve et al. [160] ; acetone/ethyl acetate (v/v 2:1) and alkane:ethyl acetate (alkane: e.g. isooctane or $n$-heptane, v/v 99.8:0.2) is applied. [161] The lipidome community has also gone out of its way to develop a comprehensive and high-throughput method for lipid analysis, which puts emphasis on simple and quick sample preparation, high resolution/sensitivity $\mathrm{MS}^{\mathrm{n}}$ analysis and data handling via extensively automated data processing. Consequently, a derivatization step for UHPLC-MS was used only once in our dataset [8] where TMSD methylation was applied for separate acidic lipid analysis; neutral and basic lipids were directly analysed after sample preparation. [8] Though derivatization may be necessary for very low-abundance lipids or to increase lipid class specificity, another reason for the reduction of sample preparation steps is the minimization of experimental errors. [137] A particularly important outlier in lipidome applications was the research on double bond position elucidation; for the absolute determination of double bond positions, other options than multiple collision $\mathrm{MS}^{\mathrm{n}}$-experiments or spectral library matching have been developed.

\section{Conclusions}

Current challenges in lipidomics are closely related to the lack of uniform methods across the field. Compared with the lipid research generally made with RP-HPLC-MS, UHPLC-MS needs more focus on the chromatographic method parameters, such as stationary (e.g. column choice, oven temperature) and mobile phase properties (flow rate, $\mathrm{pH}$, solvents, gradients, eluent modifiers).

Normalization with representative or single internal standards has been widely adopted in the field. Since computational methods alongside chemometric and statistical methods have increased in both importance and usage in HPLC-MS technology for cross-validation and dataanalysis, lipidomics needs interdisciplinary studies to reach full potential with big and complex datasets. The process of typical data-analyses in lipidomics is suggested to be reduced into four 
parts: Pre-processing, identification/quantitation, chemometric, and statistical analyses. A substantial amount of specific processing tools are provided, i.e. commercial and open-source ones, including self-made algorithms. Since ever, more lipid libraries have emerged as well for untargeted, relatively targeted and in silico methods. Currently, chemometric analyses appear to be popular in lipidomics, i.e. for developing methods, profiling lipids in samples, and evaluating metabolic relationships between lipid species. For accurate identification, ion mobility mass spectrometry has been applied with liquid chromatography, alongside chromatographic separation modes with mass-spectrometric ionization, or/and $\mathrm{MS}^{\mathrm{n}}$ systems. When chemometric methods shed light to the inter- and intra-analyte properties of typically biological medium, statistical analyses were used for data-validation (e.g. significance testing and filtering out of false positives) and detection of abonormal changes (e.g. metabolic profiling of diseases).

Funding: This research received no external funding.

Conflicts of Interest: All authors declare no conflict of interest.

\section{References}

[1] Sumner, L. W., Amberg, A., Barrett, D., Beale, M. H., Beger, R., Daykin, C. A., Fan, T. W. M., Fiehn, O., Goodacre, R., Griffin, J. L., Hankemeier, T., Hardy, N., Harnly, J., Higashi, R., Kopka, J., Lane, A. N., Lindon, J. C., Marriott, P., Nicholls, A. W., Reily, M. D., Thaden, J. J., \& Viant, M. R. (2007). Proposed minimum reporting standards for chemical analysis. Metabolomics 3(3), 211-221.

[2] Liu, M. Y., Burgos, A., Ma, L., Zhang, Q., Tang, D., \& Ruan, J. (2017). Lipidomics analysis unravels the effect of nitrogen fertilization on lipid metabolism in tea plant (Camellia sinensis L.). BMC plant biology 17(1), 165, 1-10. 
[3] Wu, J., Wu, Q., Wang, D., Kong, J., Dai, W., Wang, X., \& Yu, X. (2017). Common lipid features of lethal ventricular tarchyarrhythmias (LVTAs) induced by myocardial infarction and myocardial ion channel diseases. Scientific reports 7(1), 4220, 1-11.

[4] Dong, S., Zhang, R., Liang, Y., Shi, J., Li, J., Shang, F., Mao, X., \& Sun, J. (2017).

Changes of myocardial lipidomics profiling in a rat model of diabetic cardiomyopathy using UPLC/Q-TOF/MS analysis. Diabetology \& metabolic syndrome 9(1), 56, 1-9.

[5] Van Meulebroek, L., De Paepe, E., Vercruysse, V., Pomian, B., Bos, S., Lapauw, B., \& Vanhaecke, L. (2017). Holistic lipidomics of the human gut phenotype using validated ultrahigh-performance liquid chromatography coupled to hybrid orbitrap mass spectrometry. Analytical chemistry 89(22), 12502-12510.

[6] Ferchaud-Roucher, V., Croyal, M., Moyon, T., Zair, Y., Krempf, M., \& Ouguerram, K. (2017). Plasma lipidome analysis by liquid chromatography-high resolution mass spectrometry and ion mobility of hypertriglyceridemic patients on extended-release nicotinic acid: a pilot study. Cardiovascular drugs and therapy 31(3), 269-279.

[7] Chao, H. C., Chen, G. Y., Hsu, L. C., Liao, H. W., Yang, S. Y., Wang, S. Y., Li, Y. L., Tang, S. C., Tseng, Y. J., \& Kuo, C. H. (2017). Using precursor ion scan of 184 with liquid chromatography-electrospray ionization-tandem mass spectrometry for concentration normalization in cellular lipidomic studies. Analytica chimica acta 971, 68-77.

[8] Lee, J. W., Mok, H. J., Lee, D. Y., Park, S. C., Kim, G. S., Lee, S. E., Lee, Y.S., Kim, K. P., \& Kim, H. D. (2017). UPLC-QqQ/MS-based lipidomics approach to characterize lipid alterations in inflammatory macrophages. Journal of proteome research 16(4), 1460-1469.

[9] Triebl, A., Trötzmüller, M., Hartler, J., Stojakovic, T., \& Köfeler, H. C. (2017).

Lipidomics by ultrahigh performance liquid chromatography-high resolution mass 
spectrometry and its application to complex biological samples. Journal of Chromatography B $1053,72-80$.

[10] Cheema, A. K., Mehta, K. Y., Fatanmi, O. O., Wise, S. Y., Hinzman, C. P., Wolff, J., \& Singh, V. K. (2018). A Metabolomic and lipidomic serum signature from nonhuman primates administered with a promising radiation countermeasure, gamma-tocotrienol. International journal of molecular sciences 19(1), 79, 1-16

[11] Chen, Y., Wen, S., Jiang, M., Zhu, Y., Ding, L., Shi, H., Dong, P., Yang, J., \& Yang, Y. (2017). Atherosclerotic dyslipidemia revealed by plasma lipidomics on ApoE-/- mice fed a high-fat diet. Atherosclerosis 262, 78-86.

[12] Li, Z., Guan, M., Lin, Y., Cui, X., Zhang, Y., Zhao, Z., \& Zhu, J. (2017). Aberrant lipid metabolism in hepatocellular carcinoma revealed by liver lipidomics. International journal of molecular sciences 18(12), 2550, 1-15.

[13] Ribeiro, M. A., Murgu, M., de Moraes Silva, V., Sawaya, A. C., Ribeiro, L. F., Justi, A., \& Meurer, E. C. (2017). The screening of organic matter in mineral and tap water by UHPLC-HRMS. Talanta 174, 581-586.

[14] Bang, G., Kim, Y. H., Yoon, J., Yu, Y. J., Chung, S., \& Kim, J. A. (2017). On-Chip Lipid Extraction Using Superabsorbent Polymers for Mass Spectrometry. Analytical [22] Li, Q., Liang, X., Zhao, L., Zhang, Z., Xue, X., Wang, K., \& Wu, L. (2017). UPLC-Q-exactive orbitrap/MS-based lipidomics approach to characterize lipid extracts from bee pollen and their in vitro anti-inflammatory properties. Journal of agricultural and food chemistry $65(32)$, $6848-6860$

[15] Castro-Gómez, P., Montero, O., \& Fontecha, J. (2017). In-depth lipidomic analysis of molecular species of triacylglycerides, diacylglycerides, glycerophospholipids, and 
sphingolipids of buttermilk by GC-MS/FID, HPLC-ELSD, and UPLC-QToF-MS. International journal of molecular sciences 18(3), 605, 1-20.

[16] Lísa, M., Cífková, E., Khalikova, M., Ovčačíková, M., \& Holčapek, M. (2017).

Lipidomic analysis of biological samples: comparison of liquid chromatography, supercritical fluid chromatography and direct infusion mass spectrometry methods. Journal of Chromatography A 1525, 96-108.

[17] Patterson, R. E., Kirpich, A. S., Koelmel, J. P., Kalavalapalli, S., Morse, A. M., Cusi, K., Sunny, K., McIntyre, L. M., Garrett, T. J., \& Yost, R. A. (2017). Improved experimental data processing for UHPLC-HRMS/MS lipidomics applied to nonalcoholic fatty liver disease. Journal of Chromatography B 1002, 260-266.

[18] Lloyd, A. J., Beckmann, M., Wilson, T., Tailliart, K., Allaway, D., \& Draper, J. (2017). Ultra high performance liquid chromatography-high resolution mass spectrometry plasma lipidomics can distinguish between canine breeds despite uncontrolled environmental variability and non-standardized diets. Metabolomics 13, 15, 1-11.

[19] Chang, W. Q., Zhou, J. L., Li, Y., Shi, Z. Q., Wang, L., Yang, J., Li, P., Liu, L. F., \& Xin, G. Z. (2017). An in vitro approach for lipolysis measurement using high-resolution mass spectrometry and partial least squares based analysis. Analytica chimica acta 950, 138-146. [20] Zhang, H., Gao, Y., Sun, J., Fan, S., Yao, X., Ran, X., Zheng, C., Huang, M. \& Bi, H. (2017). Optimization of lipid extraction and analytical protocols for UHPLC-ESI-HRMSbased lipidomic analysis of adherent mammalian cancer cells. Analytical and bioanalytical chemistry 409(22), 5349-5358.

[21] Witting, M., Ruttkies, C., Neumann, S., \& Schmitt-Kopplin, P. (2017). LipidFrag: Improving reliability of in silico fragmentation of lipids and application to the Caenorhabditis elegans lipidome. PloS one 12(3), e0172311, 1-23. 
[22] Li, Q., Liang, X., Zhao, L., Zhang, Z., Xue, X., Wang, K., \& Wu, L. (2017). UPLC-Qexactive orbitrap/MS-based lipidomics approach to characterize lipid extracts from bee pollen and their in vitro anti-inflammatory properties. Journal of agricultural and food chemistry 65(32), 6848-6860.

[23] Beccaria, M., Inferrera, V., Rigano, F., Gorynski, K., Purcaro, G., Pawliszyn, J., Dugo, P., \& Mondello, L. (2017). Highly informative multiclass profiling of lipids by ultra-high performance liquid chromatography-Low resolution (quadrupole) mass spectrometry by using electrospray ionization and atmospheric pressure chemical ionization interfaces. Journal of Chromatography A 1509, 69-82.

[24] Yang, J. S., Lee, J. C., Byeon, S. K., Rha, K. H., \& Moon, M. H. (2017). Size dependent lipidomic analysis of urinary exosomes from patients with prostate cancer by flow field-flow fractionation and nanoflow liquid chromatography-tandem mass spectrometry. Analytical chemistry 89(4), 2488-2496.

[25] Blaženović, I., Shen, T., Mehta, S. S., Kind, T., Ji, J., Piparo, M., Cacciola, F., Mondello, L., \& Fiehn, O. (2018). Increasing Compound Identification Rates in Untargeted Lipidomics Research with Liquid Chromatography Drift Time-Ion Mobility Mass Spectrometry. Analytical chemistry 90(18), 10758-10764.

[26] Wu, J., Wu, Q., Dai, W., Kong, J., Lv, J., Yu, X., Wang, X., \& Wang, D. (2018). Serum lipid feature and potential biomarkers of lethal ventricular tachyarrhythmia (LVTA) induced by myocardial ion channel diseases: a rat model study. International journal of legal medicine 132(2), 439-448.

[27] De Paepe, E., Van Meulebroek, L., Rombouts, C., Huysman, S., Verplanken, K., Lapauw, B., Wauters, J., Hemeryck, L. Y., Vanhaecke, L., \& Vanhaecke, L. (2018). A validated multi-matrix platform for metabolomic fingerprinting of human urine, feces and 
plasma using ultra-high performance liquid-chromatography coupled to hybrid orbitrap highresolution mass spectrometry. Analytica chimica acta 1033, 108-118.

[28] Manni, M. M., Sot, J., Arretxe, E., Gil-Redondo, R., Falcón-Pérez, J. M., Balgoma, D., Alonso, C., Goñi, F. M., \& Alonso, A. (2018). The fatty acids of sphingomyelins and ceramides in mammalian tissues and cultured cells: Biophysical and physiological implications. Chemistry and physics of lipids 217, 29-34.

[29] Yuan, Z. X., Majchrzak-Hong, S., Keyes, G. S., Iadarola, M. J., Mannes, A. J., \& Ramsden, C. E. (2018). Lipidomic profiling of targeted oxylipins with ultra-performance liquid chromatography-tandem mass spectrometry. Analytical and bioanalytical chemistry 410(23), 6009-6029.

[30] Xuan, Q., Hu, C., Yu, D., Wang, L., Zhou, Y., Zhao, X., Li, Q, Hou, X. \& Xu, G. (2018). Development of a High Coverage Pseudotargeted Lipidomics Method Based on Ultra-High Performance Liquid Chromatography-Mass Spectrometry. International Journal of Molecular Sciences 90(12), 7608-7616.

[31] Tietz-Bogert, P., Kim, M., Cheung, A., Tabibian, J., Heimbach, J., Rosen, C., Nandakumar, M., Lazaridis, K. N., LaRusso, N. F., Sung, J. \& O’Hara, S. (2018). Metabolomic Profiling of Portal Blood and Bile Reveals Metabolic Signatures of Primary Sclerosing Cholangitis. International journal of molecular sciences 19(10), 3188, 1-19.

[32] Cheema, A., Hinzman, C., Mehta, K., Hanlon, B., Garcia, M., Fatanmi, O., \& Singh, V. (2018). Plasma Derived Exosomal Biomarkers of Exposure to Ionizing Radiation in Nonhuman Primates, International journal of molecular sciences 19(11), 3427, 1-13.

[33] Chen, Y., Ma, Z., Shen, X., Li, L., Zhong, J., Min, L. S., Xu, L., Li, H., Zhang, J., \& Dai, L. (2018). Serum lipidomics profiling to identify biomarkers for non-small cell lung cancer, Atherosclerosis 262, 78-86. 
[34] Lee, H., Choi, J. M., Cho, J. Y., Kim, T. E., Lee, H. J., \& Jung, B. H. (2018). Regulation of endogenic metabolites by rosuvastatin in hyperlipidemia patients: An integration of metabolomics and lipidomics. Chemistry and physics of lipids 214, 69-83.

[35] Shan, J., Qian, W., Shen, C., Lin, L., Xie, T., Peng, L., Xu, J., Yang, R., Ji, J., \& Zhao, X. (2018). High-resolution lipidomics reveals dysregulation of lipid metabolism in respiratory syncytial virus pneumonia mice. RSC Advances 8(51), 29368-29377.

[36] Gao, X., Luo, J., Lü, L., Zhang, L., Zhang, S., \& Cui, J. (2018). RNA-Seq and UHPLCQ-TOF/MS Based Lipidomics Study in Lysiphlebia japonica. Scientific reports 8, 7802, 1-12. [37] Yan, B., Chu, H., Yang, D., Sze, K. H., Lai, P. M., Yuan, S., Shuai, H., Wang, Y., Kao, R. Y. T., Chan, J. F. W., \& Yuen, K. Y. (2019). Characterization of the Lipidomic Profile of Human Coronavirus-Infected Cells: Implications for Lipid Metabolism Remodeling upon Coronavirus Replication. Viruses 11(1), 73, 1-16.

[38] He, Z., Wang, Y., Zhang, Y., Cheng, H., \& Liu, X. (2018). Stereoselective bioaccumulation of chiral PCB 91 in earthworm and its metabolomic and lipidomic responses. Environmental pollution 238, 421-430.

[39] Law, T. H., Volk, H. A., Pan, Y., Zanghi, B., \& Want, E. J. (2018). Metabolic perturbations associated with the consumption of a ketogenic medium-chain TAG diet in dogs with idiopathic epilepsy. British Journal of Nutrition 120(5), 484-490.

[40] Chao, Y., Gao, S., Wang, X., Li, N., Zhao, H., Wen, X., Kou, Z., \& Dong, X. (2018). Untargeted lipidomics based on UPLC-QTOF-MS/MS and structural characterization reveals dramatic compositional changes in serum and renal lipids in mice with glyoxylate-induced nephrolithiasis, Journal of Chromatography B 1095, 258-266 
[41] Lee, J. H., Yang, J. S., Lee, S. H., \& Moon, M. H. (2018), Analysis of lipoproteinspecific lipids in patients with acute coronary syndrome by asymmetrical flow field-flow fractionation and nanoflow liquid chromatography-tandem mass spectrometry. Journal of Chromatography B 1099, 56-63.

[42] Kim, S. H., Yang, J. S., Lee, J. C., Lee, J. Y., Lee, J. Y., Kim, E., \& Moon, M. H. (2018). Lipidomic alterations in lipoproteins of patients with mild cognitive impairment and Alzheimer's disease by asymmetrical flow field-flow fractionation and nanoflow ultrahigh performance liquid chromatography-tandem mass spectrometry. Journal of Chromatography A $1568,91-100$.

[43] Gobo, L. A., de Carvalho, L. M., Temp, F., Viana, C., \& Mello, C. F. (2018). A rapid method for identification and quantification of prostaglandins in cerebral tissues by UHPLCESI-MS/MS for the lipidomic in vivo studies. Analytical biochemistry 545, 98-103.

[44] Drotleff, B., Hallschmid, M., \& Lämmerhofer, M. (2018). Quantification of steroid hormones in plasma using a surrogate calibrant approach and UHPLC-ESI-QTOF-MS/MS with SWATH-acquisition combined with untargeted profiling. Analytica chimica acta 1022, $70-80$.

[45] Danne-Rasche, N., Coman, C., \& Ahrends, R. (2018). Nano-LC/NSI MS refines lipidomics by enhancing lipid coverage, measurement sensitivity, and linear dynamic range. Analytical chemistry 90(13), 8093-8101.

[46] Ulmer, C. Z., Jones, C. M., Yost, R. A., Garrett, T. J., \& Bowden, J. A. (2018). Optimization of Folch, Bligh-Dyer, and Matyash sample-to-extraction solvent ratios for human plasma-based lipidomics studies. Analytica chimica acta 1037, 351-357.

[47] Jeucken, A., \& Brouwers, J. F. (2019). High-Throughput Screening of Lipidomic Adaptations in Cultured Cells, Biomolecules 9, 42, 1-14. 
[48] Marqueño, A., Blanco, M., Maceda-Veiga, A., \& Porte, C. (2019). Skeletal muscle lipidomics as a new tool to determine altered lipid homeostasis in fish exposed to urban and industrial wastewaters. Environmental science \& technology 53, 8416-8425.

[49] Rombouts, C., De Spiegeleer, M., Van Meulebroek, L., De Vos, W. H., \& Vanhaecke, L. (2019). Validated comprehensive metabolomics and lipidomics analysis of colon tissue and cell lines. Analytica Chimica Acta 1066, 79-92.

[50] Li, J., Gao, Y., Guan, L., Zhang, H., Chen, P., Gong, X., Li, D., Liang, X., Huang, M., \& Bi, H. (2019). Lipid profiling of peri-implantation endometrium in patients with premature progesterone rise in the late follicular phase. The Journal of Clinical Endocrinology \& Metabolism 104(11), 5555-5565.

[51] Goracci, L., Valeri, A., Sciabola, S., Aleo, M. D., Moritz, W., Lichtenberg, J., \& Cruciani, G. (2019). A novel lipidomics-based approach to evaluating the risk of clinical hepatotoxicity potential of drugs in 3D human microtissues. Chemical Research in Toxicology 33, 1, 258-270

[52] Wang, X., Xu, Y., Song, X., Jia, Q., Zhang, X., Qian, Y., \& Qiu, J. (2019). Analysis of glycerophospholipid metabolism after exposure to PCB153 in PC12 cells through targeted lipidomics by UHPLC-MS/MS. Ecotoxicology and environmental safety 169, 120-127.

[53] Wang, J., Zhang, L., Xiao, R., Li, Y., Liao, S., Zhang, Z., Yang, W., \& Liang, B. (2019). Plasma lipidomic signatures of spontaneous obese rhesus monkeys. Lipids in health and disease $18,8,1-11$.

[54] Lin, M., Wang, Z., Wang, D., Chen, X., \& Zhang, J. (2019). A mathematical modelassisted UHPLC-MS/MS method for global profiling and quantification of cholesteryl esters in hyperlipidemic golden hamsters. Analytical chemistry 91, 4504-4512. 
[55] Zalloua, P., Kadar, H., Hariri, E., Farraj, L. A., Brial, F., Hedjazi, L., Lay, A. L.,

Colleum A., Dubus, J., Touboul, D., Matsuda, F., Lathrop, M., Nicholson, J. K.,Dumas, M.

E. \& Matsuda, F. (2019). Untargeted Mass Spectrometry Lipidomics identifies correlation between serum sphingomyelins and plasma cholesterol. Lipids in health and disease 18, 38, $1-10$.

[56] Kappler, L., Hoene, M., Hu, C., von Toerne, C., Li, J., Bleher, D., Hoffmann, C., Böhm, A., Kollipara, L., Zischka, H., Königsrainer, A., Häring, H. U., Peter, A., Xu, G., Sickmann, A., Hauck, S. M., Weigert, C., \& Lehmann, R. (2019). Linking bioenergetic function of mitochondria to tissue-specific molecular fingerprints. American Journal of PhysiologyEndocrinology and Metabolism 317, E374-E387.

[57] Fu, K., Wang, C., Gao, Y., Fan, S., Zhang, H., Sun, J., Jiang, Y., Liu, C., Guan, L., Liu, J., Huang, M., \& Bi, H. (2019). Metabolomics and lipidomics reveal the effect of hepatic Vps33b deficiency on bile acids and lipids metabolism. Frontiers in Pharmacology 10, 276, $1-13$.

[58] Yang, R., Zhang, Y., Qian, W., Peng, L., Lin, L., Xu, J., Xie, T., Ji, J., \& Shan, J. (2019). Surfactant Lipidomics of Alveolar Lavage Fluid in Mice Based on Ultra-High-Performance Liquid Chromatography Coupled to Hybrid Quadrupole-Exactive Orbitrap Mass Spectrometry. Metabolites 9, 80, 1-14.

[59] Creydt, M., Vuralhan-Eckert, J., Fromm, J., \& Fischer, M. (2019). Effects of elevated $\mathrm{CO} 2$ concentration on leaves and berries of black elder (Sambucus nigra) using UHPLC-ESIQTOF-MS/MS and gas exchange measurements. Journal of plant physiology 234, 71-79.

[60] Gong, L. L., Yang, S., Zhang, W., Han, F. F., Lv, Y. L., Xuan, L. L., Liu, H., \& Liu, L. H. (2019). Discovery of Metabolite Profiles of Metabolic Syndrome Using Untargeted and 
Targeted LC-MS based Lipidomics Approach. Journal of pharmaceutical and biomedical analysis $117,112848,1-8$.

[61] Chico, Y., Abad-García, B., Ochoa, B., \& Martínez, M. J. (2019). Lipidomic data uncover extensive heterogeneity in phosphatidylcholine structural variants in HepG2 cells. Data in brief 27, 104608, 1-9.

[62] Gao, X., Liu, W., Mei, J., \& Xie, J. (2019). Quantitative Analysis of Cold Stress Inducing Lipidomic Changes in Shewanella putrefaciens Using UHPLC-ESI-MS/MS. Molecules 24(24), 4609, 1-13.

[63] Chamberlain, C. A., Hatch, M., \& Garrett, T. J. (2019). Metabolomic and lipidomic characterization of Oxalobacter formigenes strains HC1 and OxWR by UHPLC-HRMS. Analytical and bioanalytical chemistry 411, 4807-4818.

[64] Liu, H., Guo, X., Zhao, Q., Qin, Y., \& Zhang, J. (2020). Lipidomics analysis for identifying the geographical origin and lactation stage of goat milk. Food chemistry 309, $125765,1-7$.

[65] Su, M., Subbaraj, A. K., Fraser, K., Qi, X., Jia, H., Chen, W., Reis, M. G., Agnew, M., Day, L., Roy, N. C., \& Young, W. (2019). Lipidomics of Brain Tissues in Rats Fed Human Milk from Chinese Mothers or Commercial Infant Formula. Metabolites 9, 253, 1-19.

[66] Calderón, C., Sanwald, C., Schlotterbeck, J., Drotleff, B., \& Lämmerhofer, M. (2019). Comparison of simple monophasic versus classical biphasic extraction protocols for comprehensive UHPLC-MS/MS lipidomic analysis of Hela cells. Analytica chimica acta 1048, 66-74.

[67] Matorras, R., Martinez-Arranz, I., Arretxe, E., Iruarrizaga-Lejarreta, M., Corral, B., Ibañez-Perez, J., ... \& Alonso, C. (2019). The lipidome of endometrial fluid differs between 
implantative and non-implantative IVF cycles. Journal of Assisted Reproduction and Genetics 37(2), 385-394.

[68] Hinz, C., Liggi, S., Mocciaro, G., Jung, S., Induruwa, I., Pereira, M., Bryant, C. E., Meckelmann, S. W., O'Donnell, V. B., Farndale, R. W., Griffin, J. L., \& Fjeldsted, J. (2019). A Comprehensive UHPLC Ion Mobility Quadrupole Time-of-Flight Method for Profiling and Quantification of Eicosanoids, Other Oxylipins, and Fatty Acids. Analytical Chemistry, $8025-8035$.

[69] King, A., Baginski, M., Morikawa, Y., Rainville, P. D., Gethings, L. A., Wilson, I. D., \& Plumb, R. S. (2019). Application of a Novel Mass Spectral Data Acquisition Approach to Lipidomic Analysis of Liver Extracts from Sitaxentan-Treated Liver-Humanized PXB Mice. Journal of proteome research 18(11), 4055-4064.

[70] Gupta, A., Dorlhiac, G. F., \& Streets, A. M. (2019). Quantitative imaging of lipid droplets in single cells. Analyst, 144(3), 753-765. nanoflow liquid chromatography-tandem mass spectrometry. Analytical chemistry 89(4), 2488-2496.

[71] Lee, G. B., Lee, J. C., \& Moon, M. H. (2019). Plasma lipid profile comparison of five different cancers by nanoflow ultrahigh performance liquid chromatography-tandem mass spectrometry. Analytica Chimica Acta 1063, 117-126.

[72] Yang, J. S., Kim, J. Y., Lee, J. C., \& Moon, M. H. (2019). Investigation of lipidomic perturbations in oxidatively stressed subcellular organelles and exosomes by asymmetrical flow field-flow fractionation and nanoflow ultrahigh performance liquid chromatographytandem mass spectrometry. Analytica chimica acta 1073, 79-89.

[73] Schlotterbeck, J., Chatterjee, M., Gawaz, M., \& Lämmerhofer, M. (2019).

Comprehensive MS/MS profiling by UHPLC-ESI-QTOF-MS/MS using SWATH data- 
independent acquisition for the study of platelet lipidomes in coronary artery disease. Analytica chimica acta 1046, 1-15.

[74] Calderón, C., Rubarth, L., Cebo, M., Merfort, I., \& Lämmerhofer, M. (2019). Lipid Atlas of Keratinocytes and Betulin Effects on its Lipidome Profiled by Comprehensive UHPLC-MS/MS With Data Independent Acquisition Using Targeted Data Processing. Proteomics (Early View), 1900113, 1-17.

[75] Drotleff, B., Illison, J., Schlotterbeck, J., Lukowski, R., \& Lämmerhofer, M. (2019).

Comprehensive Lipidomics of Mouse Plasma using Class-Specific Surrogate Calibrants and SWATH Acquisition for Large-Scale Lipid Quantification in Untargeted Analysis. Analytica Chimica Acta 1022, 70-80.

[76] Drotleff, B., \& Lämmerhofer, M. (2019). Guidelines for selection of internal standardbased normalization strategies in untargeted lipidomic profiling by LC-HR-MS/MS. Analytical chemistry 91, 9836-9843.

[77] Shan, J., Qian, W., Kang, A., Peng, L., Xie, T., Lin, L., Di, L., Xiao, P., \& Zhou, W. (2019). Lipid profile perturbations in the plasma and lungs of mice with LPS-induced acute lung injury revealed by UHPLC-ESI-Q Exactive HF MS analysis. Journal of pharmaceutical and biomedical analysis 162, 242-248.

[78] Wang, L., Li, X., Liu, L., da Zhang, H., Zhang, Y., Chang, Y. H., \& Zhu, Q. P. (2019). Comparative lipidomics analysis of human, bovine and caprine milk by UHPLC-Q-TOF-MS. Food chemistry 310, 125865, 1-8.

[79] Deng, Y., Pan, M., Nie, H., Zheng, C., Tang, K., Zhang, Y., \& Yang, Q. (2019). Lipidomic Analysis of the Protective Effects of Shenling Baizhu San on Non-Alcoholic Fatty Liver Disease in Rats. Molecules 24, 3943, 1-17. 
[80] Gross, R. W. (2017). The evolution of lipidomics through space and time. Biochimica et Biophysica Acta (BBA)-Molecular and Cell Biology of Lipids 1862(8), 731-739.

[81] Holcapek, M., Liebisch, G., \& Ekroos, K. (2018). Lipidomic Analysis, Analytical Chemistry 90, 4249-4257.

[82] Yang, K., \& Han, X. (2016). Lipidomics: techniques, applications, and outcomes related to biomedical sciences. Trends in biochemical sciences 41(11), 954-969.

[83] Hyötyläinen, T., \& Orešič, M. (2015). Optimizing the lipidomics workflow for clinical studies_-practical considerations. Analytical and bioanalytical chemistry 407(17), 49734993.

[84] Jurowski, K., Kochan, K., Walczak, J., Barańska, M., Piekoszewski, W., \& Buszewski, B. (2017). Comprehensive review of trends and analytical strategies applied for biological samples preparation and storage in modern medical lipidomics: State of the art. TrAC Trends in Analytical Chemistry 86, 276-289.

[85] Tumanov, S., \& Kamphorst, J. J. (2017). Recent advances in expanding the coverage of the lipidome. Current opinion in biotechnology 43, 127-133.

[86] Criscuolo, A., Zeller, M., Cook, K., Angelidou, G., \& Fedorova, M. (2019). Rational selection of reverse phase columns for high throughput LC-MS lipidomics. Chemistry and physics of lipids 221, 120-127.

[87] Öztaş, Y., \& Boşgelmez, İ. (2017). An Introduction to Lipidomics: From Laboratory to Clinic. Acta Medica 48(1), 14-23.

[88] Checa, A., Bedia, C., \& Jaumot, J. (2015). Lipidomic data analysis: tutorial, practical guidelines and applications. Analytica chimica acta 885, 1-16 
[89] Wakil, S. J., Stoops, J. K., \& Joshi, V. C. (1983). Fatty acid synthesis and its regulation. Regulation of Fatty Acid Synthesis Annual review of biochemistry 52(1), 537-579, p. 569.

[90] Van Meer, G., Voelker, D. R., \& Feigenson, G. W. (2008). Membrane lipids: where they are and how they behave. Nature Reviews Molecular Cell Biology 9(2), 112-124.

[91] Dennis, E. A.: "LIPID MAPS Lipid Metabolics Tutorial, Fatty Acid Biosynthesis", Powerpoint, 2010, University of California, San Diego

[92] LIPID MAPS: Tutorials and Lectures on Lipids, Categories of Lipids. 02.10.2018 http://lipidmaps.org/resources/tutorials/lipid_tutorial.php\#FA

[93] Fahy, E., Subramaniam, S., Brown, H. A., Glass, C. K., Merrill Jr, A. H., Murphy, R. C., ... \& Shimizu, T. (2005). A comprehensive classification system for lipids. European journal of lipid science and technology 107(5), 337-364.

[94] LIPID MAPS: Classification, nomenclature and structure drawing. 20.11.2018 https://www.lipidmaps.org/resources/tutorials/lipid_cns.html [95] Kim, H. S., Waqued, S. C., Nodurft, D. T., Devarenne, T. P., Yakovlev, V. V., \& Han, A. (2017). Raman spectroscopy compatible PDMS droplet microfluidic culture and analysis platform towards on-chip lipidomics. Analyst 142(7), 1054-1060.

[96] Koelmel, J. P., Kroeger, N. M., Ulmer, C. Z., Bowden, J. A., Patterson, R. E., Cochran, J. A., Beecher, C. W. W., Garrett, T. J.\& Yost, R. A. (2017). LipidMatch: an automated workflow for rule-based lipid identification using untargeted high-resolution tandem mass spectrometry data. BMC bioinformatics 18(1), 331, 1-11.

[97] Lipidomics Standards Initiative Consortium. (2019). Lipidomics needs more standardization. Nature Metabolism 1(8), 745-747. 
[98] Ovčačíková, M., Lísa, M., Cífková, E., \& Holčapek, M. (2016). Retention behavior of lipids in reversed-phase ultrahigh-performance liquid chromatography-electrospray ionization mass spectrometry. Journal of Chromatography A 1450, 76-85.

[99] Burla, B., Arita, M., Arita, M., Bendt, A. K., Cazenave-Gassiot, A., Dennis, E. A., Ekroos, K., Han, X., Ikeda, K., Liebisch, G., Lin, M. K., Loh, T. P., Meikle, P. J., Orešič, M., Quehenberger, O., Shevchenko, A., Torta, F., Wakelam, M. J. O., Wheelock, C. E., \& Wenk, M. R. (2018). MS-based lipidomics of human blood plasma: a community-initiated position paper to develop accepted guidelines. Journal of lipid research 59(10), 2001-2017. [100] Rodriguez-Mozaz, S., de Alda, M. J. L., \& Barceló, D. (2007). Advantages and limitations of on-line solid phase extraction coupled to liquid chromatography-mass spectrometry technologies versus biosensors for monitoring of emerging contaminants in water. Journal of chromatography A 1152(1-2), 97-115.

[101] Avela, H. F. \& Sirén, H. (2020) Advances in lipidomics, Clinica Chimica Acta. (in-print) [102] Kitov, P. I., Han, L., Kitova, E. N., \& Klassen, J. S. (2019). Sliding window adduct removal method (SWARM) for enhanced electrospray ionization mass spectrometry binding data. Journal of The American Society for Mass Spectrometry 30(8), 1446-1454.

[103] Gorrochategui, E., Jaumot, J., Lacorte, S., \& Tauler, R. (2016). Data analysis strategies for targeted and untargeted LC-MS metabolomic studies: Overview and workflow. TrAC Trends in Analytical Chemistry 82, 425-442.

[104] Lam, S. M., Tian, H., \& Shui, G. (2017). Lipidomics, en route to accurate quantitation. Biochimica et Biophysica Acta (BBA)-Molecular and Cell Biology of Lipids 1862(8), 752761. 
[105] Vu, N., Narvaez-Rivas, M., Chen, G. Y., Rewers, M. J., \& Zhang, Q. (2019). Accurate mass and retention time library of serum lipids for type 1 diabetes research. Analytical and bioanalytical chemistry 411(23), 5937-5949.

[106] Bielow, C., Mastrobuoni, G. Orioli, M., \& Kempa, S. (2017). On mass ambiguities in high-resolution shotgun lipidomics. Analytical chemistry, 89(5), 2986-2994.

[107] Dong, M. W. (2013), Myths in ultrahigh-pressure liquid chromatography, LCGC North America 31(10), 868-880

[108] Avela, H. F. (2019). Recent Developments and Challenges in Chromatographic Lipidomics. Helsinki University, E-thesis

[109] Cífková, E., Hájek, R., Lísa, M., \& HolLapek, M. (2016). Hydrophilic interaction liquid chromatography mass spectrometry of (lyso) phosphatidic acids,(lyso) phosphatidylserines and other lipid classes. Journal of Chromatography A 1439, 65-73. [110] Moldoveanu, S. C., \& David, V. (2016). Selection of the HPLC method in chemical analysis. Elsevier, ISBN:978-0-12-803684-6, 40-42

[111] Brouwers, J. F. (2011). Liquid chromatographic-mass spectrometric analysis of phospholipids. Chromatography, ionization and quantification. Biochimica et Biophysica Acta (BBA)-Molecular and Cell Biology of Lipids 1811(11), 763-775.

[112] Cajka, T., \& Fiehn, O. (2014). Comprehensive analysis of lipids in biological systems by liquid chromatography-mass spectrometry. TrAC Trends in Analytical Chemistry 61, 192206.

[113] Triebl, A., Trötzmüller, M., Eberl, A., Hanel, P., Hartler, J., \& Köfeler, H. C. (2014). Quantitation of phosphatidic acid and lysophosphatidic acid molecular species using hydrophilic interaction liquid chromatography coupled to electrospray ionization high resolution mass spectrometry. Journal of chromatography A 1347, 104-110. 
[114] Rozing, G. (2003). Trends in HPLC column formats-Microbore, nanobore and smaller. LC GC EUROPE 16(6 A), 14-19.

[115] Storey, J. D. (2003). The positive false discovery rate: a Bayesian interpretation and the q-value. The Annals of Statistics 31(6), 2013-2035.

[116] Mannur, V. S., Patel, D., Mastiholimath, V. S., \& Shah, G. (2011). Selection of buffers in LC-MS/MS: an overview. International Journal of Pharmaceutical Science s Review and Research 6(1), Article-008, 35-37.

[117] Cajka, T., \& Fiehn, O. (2016). Toward merging untargeted and targeted methods in mass spectrometry-based metabolomics and lipidomics. Analytical chemistry 88(1), 524-545.

[118] Monnin, C., Ramrup, P., Daigle-Young, C., \& Vuckovic, D. (2018). Improving negative liquid chromatography/electrospray ionization mass spectrometry lipidomic analysis of human plasma using acetic acid as a mobile-phase additive. Rapid Communications in Mass Spectrometry 32(3), 201-211.

[119] Zhao, X., Zhang, W., Zhang, D., Liu, X., Cao, W., Chen, Q., Ouyang, Z., \& Xia, Y. (2019). A lipidomic workflow capable of resolving sn-and C [double bond, length as m-dash] C location isomers of phosphatidylcholines. Chemical Science 10(46), 10740-10748.

[120] Xiao, Y.J.; Schwartz, B.; Washington, M.; Kennedy, A.; Webster, K.; Belinson, J.; Xu, Y. (2001). Electrospray ionization mass spectrometry analysis of lysophospholipids in human ascitic fluids: Comparison of the lysophospholipid contents in malignant vs. nonmalignant ascitic fluids. Analytical Biochemistry 290, 302-313.

[121] Lee, D. Y., Kind, T., Yoon, Y. R., Fiehn, O., \& Liu, K. H. (2014). Comparative evaluation of extraction methods for simultaneous mass-spectrometric analysis of complex 
lipids and primary metabolites from human blood plasma. Analytical and bioanalytical chemistry 406(28), 7275-7286.

[122] Taylor, N., White, T., \& Viant, M. (2017). Defining the baseline and oxidant perturbed lipidomic profiles of daphnia magna. Metabolites 7, 11, 1-14.

[123] Byeon, S. K., Kim, J. Y., Lee, J. Y., Chung, B. C., Seo, H. S., \& Moon, M. H. (2015). Top-down and bottom-up lipidomic analysis of rabbit lipoproteins under different metabolic conditions using flow field-flow fractionation, nanoflow liquid chromatography and mass spectrometry. Journal of Chromatography A 1405, 140-148.

[124] Zhang, J., Rector, J., Lin, J. Q., Young, J. H., Sans, M., Katta, N., Giese, N., Yu, W., Nagi, C., Suliburk, J., Liu, J., Bensussan, A.m DeHoog, R. J., Garza, K. Y., Ludolph, B., Sorace, A. G.,, Syed, A., Zahedivash, A., Milner, T. E., \& Eberlin, L. S. (2017). Nondestructive tissue analysis for ex vivo and in vivo cancer diagnosis using a handheld mass spectrometry system. Science translational medicine 9(406), eaan3968.

[125] Law, K. P., \& Zhang, C. L. (2019). Current Progress and Future Trends of Mass Spectrometry-based Archaeal Lipidomics. Organic geochemistry 134, 45-61.

[126] Vasilopoulou, C. G., Sulek, K., Brunner, A. D., Meitei, N. S., Schweiger-Hufnagel, U., Meyer, S. W., Barsch, A., Mann, M. \& Meier, F. (2020). Trapped ion mobility spectrometry and PASEF enable in-depth lipidomics from minimal sample amounts. Nature Communications, 11(1), 1-11.

[127] Sethi, S., \& Brietzke, E. (2017). Recent advances in lipidomics: Analytical and clinical perspectives. Prostaglandins \& other lipid mediators 128, 8-16.

[128] Smith, R., Mathis, A. D., Ventura, D., \& Prince, J. T. (2014). Proteomics, lipidomics, metabolomics: a mass spectrometry tutorial from a computer scientist's point of view. BMC Bioinformatics 15(Suppl 7), S9, 1-14. 
[129] Cech, N. B., \& Enke, C. G. (2001). Practical implications of some recent studies in electrospray ionization fundamentals. Mass spectrometry reviews, 20(6), 362-387.

[130] Pham, H. T., Trevitt, A. J., Mitchell, T. W., \& Blanksby, S. J. (2013). Rapid differentiation of isomeric lipids by photodissociation mass spectrometry of fatty acid derivatives. Rapid Communications in Mass Spectrometry 27(7), 805-815.

[131] Song, C., Gao, D., Li, S., Liu, L., Chen, X., \& Jiang, Y. (2019). Determination and quantification of fatty acid $\mathrm{C}=\mathrm{C}$ isomers by epoxidation reaction and liquid chromatographymass spectrometry. Analytica Chimica Acta 1086, 82-89.

[132] Murphy, R. C., Okuno, T., Johnson, C. A., \& Barkley, R. M. (2017). Determination of double bond positions in polyunsaturated fatty acids using the photochemical Paterno-Buchi reaction with acetone and tandem mass spectrometry. Analytical chemistry 89(16), 85458553.

[133] Smilowitz, J. T., Zivkovic, A. M., Wan, Y. J. Y., Watkins, S. M., Nording, M. L., Hammock, B. D., \& German, J. B. (2013). Nutritional lipidomics: molecular metabolism, analytics, and diagnostics. Molecular nutrition \& food research, 57(8), 1319-1335.

[134] Deng, J., Yang, Y., Liu, Y., Fang, L., Lin, L., \& Luan, T. (2019). Coupling PaternòBüchi Reaction with Surface-Coated Probe Nanoelectrospray Ionization Mass Spectrometry for In Vivo and Microscale Profiling of Lipid C $=\mathrm{C}$ Location Isomers in Complex Biological Tissues. Analytical chemistry 91(7), 4592-4599.

[135] Castro-Perez, J., Roddy, T. P., Nibbering, N. M., Shah, V., McLaren, D. G., Previs, S., Attygalle, A. B., Herath, K., Chen, Z., Wang, S.-P., Mitnaul, L., Hubbard, B. K., Vreeken, R. J., Johns, D. G., \& Hankemeier, T. (2011). Localization of fatty acyl and double bond positions in phosphatidylcholines using a dual stage CID fragmentation coupled with ion mobility mass spectrometry. Journal of the American Society for Mass Spectrometry, 22(9), 1552-1567. 
[136] Lísa, M., Netušilová, K., Franěk, L., Dvořáková, H., Vrkoslav, V., \& Holčapek, M. (2011). Characterization of fatty acid and triacylglycerol composition in animal fats using silver-ion and non-aqueous reversed-phase high-performance liquid chromatography/mass spectrometry and gas chromatography/flame ionization detection. Journal of Chromatography A $1218(42), 7499-7510$.

[137] Yang, K., \& Han, X. (2011). Accurate quantification of lipid species by electrospray ionization mass spectrometry — meets a key challenge in lipidomics. Metabolites, 1(1), 21-40. [138] Rampler, E., Coman, C., Hermann, G., Sickmann, A., Ahrends, R., \& Koellensperger, G. (2017). LILY-lipidome isotope labeling of yeast: in vivo synthesis of 13 C labeled reference lipids for quantification by mass spectrometry. Analyst 142(11), 1891-1899.

[139] Boysen, A. K., Heal, K. R., Carlson, L. T., \& Ingalls, A. E. (2018). Best-matched internal standard normalization in liquid chromatography-mass spectrometry metabolomics applied to environmental samples. Analytical chemistry, 90(2), 1363-1369.

[140] Lennart Eriksson, Sartorius Stedim Biotech, Umetrics Suite blog - "OPLS vs PCA: Explaining differences or grouping data? “ https://blog.umetrics.com/explaining-differencesor-grouping-data-opls-da-vs-pca-data-analysis, page checked 24.06.2019

[141] Kelechi, A. C. (2012). Regression and principal component analysis: comparison using few regressors. American Journal of Mathematics and Statistics 2(1), 1-5.

[142] Wold, S., Ruhe, A., Wold, H., \& Dunn, III, W. J. (1984). The collinearity problem in linear regression. The partial least squares (PLS) approach to generalized inverses. SIAM Journal on Scientific and Statistical Computing 5(3), 735-743.

[143] Holme, P., Huss, M., \& Jeong, H. (2003). Subnetwork hierarchies of biochemical pathways. Bioinformatics 19(4), 532-538. 
[144] Kahler, E. G. F. T. (2016). Effects of Column Inner Diameter and Packed Bed [145] Benjamini, Y., \& Hochberg, Y. (1995). Controlling the false discovery rate: a practical and powerful approach to multiple testing. Journal of the Royal statistical society: series B (Methodological) 57(1), 289-300.

[146] Ruxton, G. D. (2006). The unequal variance t-test is an underused alternative to Student's t-test and the Mann-Whitney U test. Behavioral Ecology 17(4), 688-690.

[147] Nachar, N. (2008). The Mann-Whitney U: A test for assessing whether two independent samples come from the same distribution. Tutorials in quantitative Methods for Psychology 4(1), 13-20.

[148] Svan, A., Hedeland, M., Arvidsson, T., \& Pettersson, C. E. (2018). The differences in matrix effect between supercritical fluid chromatography and reversed phase liquid chromatography coupled to ESI/MS. Analytica chimica acta 1000, 163-171.

[149] Gergov, M., Nenonen, T., Ojanperä, I., \& Ketola, R. A. (2015). Compensation of matrix effects in a standard addition method for metformin in postmortem blood using liquid chromatography-electrospray-tandem mass spectrometry. Journal of analytical toxicology, 39(5), 359-364.

[150] Dolan, J. W. (2002). The importance of temperature. LC GC NORTH AMERICA 20(6), 524-531.

[151] Dolan, J. (2016). A guide to HPLC and LC-MS buffer selection. ACE HPLC Columnsultra inert base-deactivated HPLC columns, Advanced Chromatography Technologies (Aberdeen, Scotland), 1-20.

[152] Sasaki, H., Arai, H., Cocco, M. J., \& White, S. H. (2009). pH dependence of sphingosine aggregation. Biophysical journal 96(7), 2727-2733. 
[153] Tsui, F. C., Ojcius, D. M., \& Hubbell, W. L. (1986). The intrinsic pKa values for phosphatidylserine and phosphatidylethanolamine in phosphatidylcholine host bilayers. Biophysical journal 49(2), 459-468.

[154] Konermann, L. (2017). Addressing a common misconception: Ammonium acetate as neutral $\mathrm{pH}$ "buffer" for native electrospray mass spectrometry. Journal of the American Society for Mass Spectrometry 28, 1827-1835.

[155] Aristizabal, H. J. J., Bradley, R. M., Duncan, R. E., \& Stark, K. D. (2018) Categorizing and qualifying nutritional lipidomic data: defining brutto, medio, genio and infinio lipid species within macrolipidomics and microlipidomics. Current Opinion in Clinical Nutrition and Metabolic Care 21(5), 352-359.

[156] Holčapek, M., Liebisch, G., \& Ekroos, K. (2020). Lipidomic analysis. Analytical and Bioanalytical Chemistry 412, 2187-2189.

[157] Touboul, D., \& Ollero, M. (2019). Lipidomics Conquers a Niche, Consolidates Growth. International Journal Molecular Sciences 20(13), 3188, 1-4.

[158] Ibáñez, C., Mouhid, L., Reglero, G., \& Ramírez de Molina, A. (2017). Lipidomics insights in health and nutritional intervention studies. Journal of agricultural and food chemistry, 65(36), 7827-7842.

[159] de Castro, M. L., \& Quiles-Zafra, R. (2020). Lipidomics: an omics discipline with a key role in nutrition. Talanta 219, article 121197.

[160] Graeve, M., \& Janssen, D. (2009). Improved separation and quantification of neutral and polar lipid classes by HPLC-ELSD using a monolithic silica phase: application to exceptional marine lipids. Journal of Chromatography B 877(20-21), 1815-1819.

[161] Imbert, L., Gaudin, M., Libong, D., Touboul, D., Abreu, S., Loiseau, P. M., ... \& Chaminade, P. (2012). Comparison of electrospray ionization, atmospheric pressure chemical 
ionization and atmospheric pressure photoionization for a lipidomic analysis of Leishmania donovani. Journal of Chromatography A 1242, 75-83. 
Supplementary Table S1. Experimental conditions for the solid and mobile phase in chromatography

\begin{tabular}{|c|c|c|c|c|c|c|c|}
\hline Precolumn & Column & Polar Solvent (v/v ratios) & Organic Solvent (v/v ratios) & Solvent C & Additives & Year & Citation \\
\hline \multirow[t]{26}{*}{ Yes } & T3 C18 & 1:1 MeOH: $\mathrm{H}_{2} \mathrm{O}(\mathrm{pH} 7.5)$ (PG;PS) & 6:4 MeOH:ACN (GP;PS) & & AmFo, FoA & 2017 & {$[15]$} \\
\hline & C18 HSS & 19:19:2 ACN:MeOH: $\mathrm{H}_{2} \mathrm{O}$ & IPA & & AmFo, FoA & 2017 & [8] \\
\hline & C18 HSS T3 & 3:4:3 ACN:acetone:IPA (DG;TG) & 3:7 ACN:IPA (DG;TG) & & $\mathrm{AmOH}$ & 2017 & {$[15]$} \\
\hline & C18 BEH & $40: 60 \mathrm{ACN}: \mathrm{H}_{2} \mathrm{O}$ & 90:10 IPA:ACN & & AmAc, AcA & 2017 & [19] \\
\hline & $\mathrm{N} / \mathrm{A}$ & 40:60 ACN: $\mathrm{H}_{2} \mathrm{O}$ & 90:10 IPA:ACN & & AmAc & 2017 & {$[18]$} \\
\hline & $\mathrm{C} 18$ & $50: 50 \mathrm{ACN}: \mathrm{H}_{2} \mathrm{O}$ & 95:5 IPA:ACN & & AmFo, FoA & 2017 & {$[20]$} \\
\hline & $\mathrm{C} 18 \mathrm{CSH}$ & $\mathrm{H}_{2} \mathrm{O}$ & 50:50 IPA:ACN & & AmFo, FoA & 2017 & {$[11]$} \\
\hline & $\mathrm{C} 18$ & $60: 40 \mathrm{MeOH} / \mathrm{H}_{2} \mathrm{O}$ & 60:40 MeOH:IPA & & AmAc, AcA & 2017 & [7] \\
\hline & $\mathrm{C} 18$ & $60: 40 \mathrm{ACN}: \mathrm{H}_{2} \mathrm{O}$ & 90:10 IPA:ACN & & AmAc & 2017 & {$[22]$} \\
\hline & C18 & $60: 40 \mathrm{ACN}: \mathrm{H}_{2} \mathrm{O}$ & 90:10 IPA:ACN & & AmFo, FoA & 2017 & {$[21]$} \\
\hline & $\mathrm{C} 18 \mathrm{CSH}$ & $60: 40 \mathrm{ACN}: \mathrm{H}_{2} \mathrm{O}$ & 90:10 IPA:ACN & & - & 2017 & [4] \\
\hline & $\mathrm{C} 18 \mathrm{CSH}$ & $60: 40 \mathrm{ACN}: \mathrm{H}_{2} \mathrm{O}$ & 90:10 IPA:ACN & & AmAc, FoA & 2017 & {$[6]$} \\
\hline & $\mathrm{C} 18 \mathrm{CSH}$ & $60: 40 \mathrm{ACN}: \mathrm{H}_{2} \mathrm{O}$ & $90: 10$ IPA:ACN & & AmAc & 2017 & {$[22]$} \\
\hline & $\mathrm{C} 18 \mathrm{CSH}$ & $60: 40 \mathrm{ACN}: \mathrm{H}_{2} \mathrm{O}$ & 90:10 IPA:ACN & & AmFo, FoA & 2017 & {$[14]$} \\
\hline & C18 HSS & $60: 40 \mathrm{ACN}: \mathrm{H}_{2} \mathrm{O}$ & 90:10 IPA:ACN & & AmFo & 2017 & [3] \\
\hline & C18 BEH & $90: 10 \mathrm{ACN}: \mathrm{H}_{2} \mathrm{O}$ & $90: 80: 1$ IPA:ACN: $\mathrm{H}_{2} \mathrm{O}$ & & AmFo, FoA & 2017 & {$[17]$} \\
\hline & C18 BEH* & $90: 10 \mathrm{H}_{2} \mathrm{O}: \mathrm{ACN}$ & 20:20:60 MeOH:ACN:IPA & & AmFo, FoA & 2017 & {$[24]^{*}$} \\
\hline & C18 HSS T3 & 90:10 IPA:ACN & $30: 70 \mathrm{H}_{2} \mathrm{O}: \mathrm{ACN}$ & & AmFo & 2017 & {$[12]$} \\
\hline & HILIC, BEH & $\mathrm{H}_{2} \mathrm{O}$ & $96: 4 \mathrm{ACN}: \mathrm{H}_{2} \mathrm{O}$ & & AmAc & 2017 & [16] \\
\hline & $\mathrm{C} 8 \mathrm{BEH}$ & $\mathrm{H}_{2} \mathrm{O}$ & 2:5 IPA:ACN & & AmFo, FoA & 2017 & [9] \\
\hline & C18 BEH & $\mathrm{H}_{2} \mathrm{O}$ & 50:50 IPA:ACN & & - & 2017 & [19] \\
\hline & $\mathrm{C} 18^{* *}$ & $\mathrm{H}_{2} \mathrm{O}$ & 60:36:4 IPA:ACN: $\mathrm{H}_{2} \mathrm{O}$ & & AmFo & 2017 & {$[23]^{* *}$} \\
\hline & $\mathrm{C} 8$ & $\mathrm{H}_{2} \mathrm{O}$ & 70:30 ACN:IPA & & AmAc, FoA & 2017 & {$[2]$} \\
\hline & C18 BEH & $\mathrm{H}_{2} \mathrm{O}$ & $\mathrm{ACN}$ & 90:10 IPA:ACN & FoA & 2017 & [10] \\
\hline & C18 HSS T3 & $\mathrm{H}_{2} \mathrm{O}$ & $\mathrm{ACN}$ & & FoA & 2017 & [13] \\
\hline & C18 BEH & $\mathrm{H}_{2} \mathrm{O}$ & $\mathrm{MeOH}$ & & AmAc & 2017 & {$[5]$} \\
\hline
\end{tabular}

*nano-LC, **APCl instead of ESI, ***narrow-bore UHPLC

BEH: ethylene bridged hybrid, CSH: charged surface hybrid, HSS: high strength silica, SB: stable bond packing 


\begin{tabular}{|c|c|c|c|c|c|c|c|}
\hline Precolumn & Column & Polar Solvent (v/v ratios) & Organic Solvent (v/v ratios) & Solvent C & Additives & Year & Citation \\
\hline & $\mathrm{C} 18 \mathrm{CSH}$ & 40:60 ACN: $\mathrm{H}_{2} \mathrm{O}$ & 90:10 IPA:ACN & & AmFo, FoA & 2018 & {$[35]$} \\
\hline & C18 & 45:55 ACN: $\mathrm{H}_{2} \mathrm{O}$ & & & AcA & 2018 & [43] \\
\hline & C18 BEH & $60: 40$ ACN: $\mathrm{H}_{2} \mathrm{O}$ & $81: 10: 9$ IPA:ACN: $\mathrm{H}_{2} \mathrm{O}$ & & AmFo, FoA & 2018 & {$[40]$} \\
\hline & $\mathrm{C} 18 \mathrm{CSH}$ & $60: 40$ ACN: $\mathrm{H}_{2} \mathrm{O}$ & 90:10 ACN:IPA & & AmFo, FoA & 2018 & [39] \\
\hline & C18 & $60: 40$ ACN: $\mathrm{H}_{2} \mathrm{O}$ & 90:10 IPA:ACN & & AmFo & 2018 & [33] \\
\hline & C18 & $60: 40$ ACN: $\mathrm{H}_{2} \mathrm{O}$ & 90:10 IPA:ACN & & $A m A c$ & 2018 & [38] \\
\hline \multirow[t]{2}{*}{ Yes } & C18 CSH & $60: 40 \mathrm{ACN}: \mathrm{H}_{2} \mathrm{O}$ & 90:10 IPA:ACN & & AmFo, FoA & 2018 & {$[25]$} \\
\hline & C18 HSS & $60: 40$ ACN: $\mathrm{H}_{2} \mathrm{O}$ & 90:10 IPA:ACN & & AmFo & 2018 & {$[26]$} \\
\hline Yes & C18* & $60: 40$ ACN: $\mathrm{H}_{2} \mathrm{O}$ & 90:10 IPA:ACN & & AmFo, FoA & 2018 & {$[45]^{*}$} \\
\hline \multirow[t]{5}{*}{ Yes } & $\mathrm{C} 18^{* * *}$ & $60: 40$ ACN: $\mathrm{H}_{2} \mathrm{O}$ & 90:10 IPA:ACN & & AmFo, FoA & 2018 & {$[45]^{* * *}$} \\
\hline & C8 BEH & $60: 40$ ACN: $\mathrm{H}_{2} \mathrm{O}$ & 90:10 IPA:ACN & & AmAc & 2018 & [30] \\
\hline & C8 BEH & $60: 40$ ACN: $\mathrm{H}_{2} \mathrm{O}$ & 90:10 IPA:ACN & & AmAc & 2018 & {$[30]$} \\
\hline & C18 BEH & $60: 40$ ACN: $\mathrm{H}_{2} \mathrm{O}$ & 90:10 IPA:ACN & & AmFo & 2018 & [28] \\
\hline & C18 BEH & $60: 40$ ACN: $\mathrm{H}_{2} \mathrm{O}$ & $90: 8: 2$ IPA:ACN: $\mathrm{H}_{2} \mathrm{O}$ & & AmFo, FoA & 2018 & [46] \\
\hline Yes & C18 BEH* & $90: 10 \mathrm{H}_{2} \mathrm{O}: \mathrm{ACN}$ & 20:20:60 MeOH:ACN:IPA & & AmFo, FoA & 2018 & {$[41]^{*}$} \\
\hline \multirow[t]{12}{*}{ Yes } & C18 BEH* & $90: 10 \mathrm{H}_{2} \mathrm{O}: \mathrm{ACN}$ & 20:20:60 MeOH:ACN:IPA & & AmFo, FoA & 2018 & {$[42]^{*}$} \\
\hline & C18 SB & $\mathrm{H}_{2} \mathrm{O}$ & $90: 10 \mathrm{ACN}: \mathrm{H}_{2} \mathrm{O}$ & & AmAc, AcA & 2018 & [29] \\
\hline & $\mathrm{C} 18^{* *}$ & $\mathrm{H}_{2} \mathrm{O}$ & ACN & & FoA & 2018 & {$[44]^{* *}$} \\
\hline & C18 BEH & $\mathrm{H}_{2} \mathrm{O}$ & $\mathrm{ACN}$ & $90: 10$ IPA:ACN & FoA & 2018 & [32] \\
\hline & C18 BEH & $\mathrm{H}_{2} \mathrm{O}$ & $\mathrm{ACN}$ & & AcA & 2018 & [37] \\
\hline & C18 HSS & $\mathrm{H}_{2} \mathrm{O}$ & $\mathrm{ACN}$ & & FoA & 2018 & {$[27]$} \\
\hline & Amide & $\mathrm{H}_{2} \mathrm{O}$ & $\mathrm{MeOH}$ & & $\mathrm{AmHCO} 3$ & 2018 & [31] \\
\hline & Amide & $\mathrm{H}_{2} \mathrm{O}$ & $\mathrm{MeOH}$ & & AmFo & 2018 & [31] \\
\hline & C18 BEH & $\mathrm{H}_{2} \mathrm{O}$ & $\mathrm{MeOH}$ & & PFPA, FoA & 2018 & [31] \\
\hline & C18 BEH & $\mathrm{H}_{2} \mathrm{O}$ & $\mathrm{MeOH}$ & & PFPA, FoA & 2018 & [31] \\
\hline & $\mathrm{C} 18 \mathrm{CSH}$ & $\mathrm{H}_{2} \mathrm{O}$ & $\mathrm{MeOH}$ & & FoA & 2018 & [34] \\
\hline & C18 HSS & $\mathrm{H}_{2} \mathrm{O}$ & $\mathrm{MeOH}$ & & FoA & 2018 & [39] \\
\hline
\end{tabular}

*nano-LC, ${ }^{* *}$ APCl instead of ESI, ***narrow-bore UHPLC

BEH: ethylene bridged hybrid, CSH: charged surface hybrid, HSS: high strength silica, SB: stable bond packing 


\begin{tabular}{|c|c|c|c|c|c|c|c|}
\hline Precolumn & Column & Polar Solvent ( $\mathrm{v} / \mathrm{v}$ ratios) & Organic Solvent (v/v ratios) & Solvent C & Additives & Year & Citation \\
\hline & C18 BEH* & 10:90 $\mathrm{H}_{2} \mathrm{O}: \mathrm{ACN}$ & 20:20:60 MeOH:ACN:IPA & & AmFo, NH3 & 2019 & [71] \\
\hline & $\mathrm{C} 18 \mathrm{CSH}$ & $40: 60 \mathrm{ACN}: \mathrm{H}_{2} \mathrm{O}$ & 90:10 IPA:ACN & & AmFo, FoA & 2019 & [77] \\
\hline & C18 & $50: 50 \mathrm{ACN}: \mathrm{H}_{2} \mathrm{O}$ & 20:80 IPA:MeOH & & $A m A c$ & 2019 & {$[52]$} \\
\hline & C18 & 50:50 H2O:ACN & 5:95 ACN:IPA & & AmFo, FoA & 2019 & {$[50]$} \\
\hline & C18 & 50:50 H2O:ACN & 5:95 ACN:IPA & & AmFo, FoA & 2019 & [57] \\
\hline \multirow[t]{3}{*}{ Yes } & $\mathrm{C} 18 \mathrm{CSH}$ & $60: 40 \mathrm{ACN}: \mathrm{H}_{2} \mathrm{O}$ & $90: 10 \mathrm{ACN}: \mathrm{H}_{2} \mathrm{O}$ & & AmFo, FoA & 2019 & [55] \\
\hline & C18 HSS T3 & 60:40 ACN:H2O & 90:10 ACN:IPA & & AmFo, FoA & 2019 & {$[60]$} \\
\hline & $\mathrm{C} 18 \mathrm{CSH}$ & $60: 40$ ACN:H2O & 90:10 ACN:IPA & & AmFo, FoA & 2019 & {$[60]$} \\
\hline \multirow[t]{10}{*}{ Yes } & $\mathrm{C} 18 \mathrm{CSH}$ & $60: 40 \mathrm{ACN}: \mathrm{H}_{2} \mathrm{O}$ & 90:10 IPA:ACN & & AmFo, FoA & 2019 & {$[66]$} \\
\hline & $\mathrm{C} 18 \mathrm{CSH}$ & $60: 40$ ACN:H2O & 90:10 IPA:ACN & & AmFo, FoA & 2019 & [74] \\
\hline & $\mathrm{C} 8$ & $60: 40$ ACN:H2O & 90:10 IPA:ACN & & $A m A c$ & 2019 & {$[56]$} \\
\hline & $\mathrm{C} 18 \mathrm{CSH}$ & $60: 40$ ACN:H2O & 90:10 IPA:ACN & & AmFo, FoA & 2019 & {$[65]$} \\
\hline & $\mathrm{C} 18 \mathrm{CSH}$ & 60:40 ACN:H2O & 90:10 IPA:ACN & & AmFo & 2019 & [79] \\
\hline & C18 & $60: 40$ ACN:H2O & 90:10 IPA:ACN & & AmFo & 2019 & [78] \\
\hline & $\mathrm{C} 18 \mathrm{CSH}$ & $60: 40$ ACN:H2O & $90: 10$ IPA:ACN & & AmFo, FoA & 2019 & [69] \\
\hline & $\mathrm{C} 18 \mathrm{CSH}$ & $60: 40$ ACN:H2O & 90:10 IPA:ACN & & AmFo & 2019 & {$[62]$} \\
\hline & $\mathrm{C} 18 \mathrm{CSH}$ & 60:40 ACN:H2O & 90:10 IPA:ACN & & AmAc & 2020 & [64] \\
\hline & $\mathrm{C} 18 \mathrm{CSH}$ & $60: 40$ ACN:H2O & 90:10 IPA:ACN & & AmAc & 2020 & {$[64]$} \\
\hline Yes & C18 BEH & $60: 40 \mathrm{ACN}: \mathrm{H}_{2} \mathrm{O}$ & $90: 8: 2$ IPA:ACN: $\mathrm{H}_{2} \mathrm{O}$ & & AmFo, FoA & 2019 & {$[63]$} \\
\hline Yes & $\mathrm{C} 18 \mathrm{CSH}$ & 60:40 ACN:H2O & 90:9:1 IPA:ACN:H2O & & AmFo, FoA & 2019 & [75] \\
\hline \multirow[t]{6}{*}{ Yes } & $\mathrm{C} 18 \mathrm{CSH}$ & $60: 40$ ACN:H2O & 90:9:1 IPA:ACN:H2O & & AmFo, FoA & 2019 & [76] \\
\hline & $\mathrm{C} 18 \mathrm{CSH}$ & 60:40 H2O:ACN & 90:10 IPA:ACN & & AmFo, FoA & 2019 & {$[58]$} \\
\hline & HILIC & $70: 30 \mathrm{ACN}: \mathrm{H}_{2} \mathrm{O}$ & 90:10 ACN:acetone & & AmFo, FoA & 2019 & [47] \\
\hline & HILIC** & $70: 30 \mathrm{ACN}: \mathrm{H}_{2} \mathrm{O}$ & 90:10 ACN:acetone & & AmFo, FoA & 2019 & {$[47]^{* *}$} \\
\hline & C18 BEH & $\mathrm{ACN}: \mathrm{H} 2 \mathrm{O}$ & IPA:ACN & & AmFo & 2019 & [67] \\
\hline & $\mathrm{C} 18 \mathrm{CSH}$ & $\mathrm{H} 2 \mathrm{O}$ & 16:3 ACN:MeOH & & AcA & 2019 & [68] \\
\hline
\end{tabular}

*nano-LC, ${ }^{* *} \mathrm{APCl}$ instead of ESI, ***narrow-bore UHPLC

BEH: ethylene bridged hybrid, CSH: charged surface hybrid, HSS: high strength silica, SB: stable bond packing 


\begin{tabular}{|c|c|c|c|c|c|c|c|}
\hline Precolumn & Column & Polar Solvent ( $v / v$ ratios) & Organic Solvent (v/v ratios) & Solvent C & Additives & Year & Citation \\
\hline \multirow[t]{7}{*}{ Yes } & $\mathrm{C} 8$ & $\mathrm{H}_{2} \mathrm{O}$ & 55:40:5 ACN:IPA: $\mathrm{H}_{2} \mathrm{O}$ & & $A m A c$ & 2019 & {$[68]$} \\
\hline & C18 & $\mathrm{H}_{2} \mathrm{O}$ & 75:25 IPA:ACN & & AmFo & 2019 & [59] \\
\hline & C18 & $\mathrm{H}_{2} \mathrm{O}$ & 75:25 IPA:ACN & & AmAc & 2019 & [59] \\
\hline & C18 BEH & $\mathrm{H} 2 \mathrm{O}$ & $\mathrm{ACN}$ & & FoA & 2019 & [67] \\
\hline & F5 & $\mathrm{H} 2 \mathrm{O}$ & IPA & & AmFo, FoA & 2019 & {$[51]$} \\
\hline & C18 & $\mathrm{H}_{2} \mathrm{O}$ & $\mathrm{MeOH}$ & & AmAc, FoA & 2019 & {$[53]$} \\
\hline & C18 & $\mathrm{H}_{2} \mathrm{O}$ & $\mathrm{MeOH}$ & & AmAc, FoA & 2019 & [53] \\
\hline \multirow[t]{4}{*}{ Yes } & Phenyl & $\mathrm{H}_{2} \mathrm{O}$ & $\mathrm{MeOH}$ & & $A m A c$ & 2019 & [49] \\
\hline & C8 BEH & $\mathrm{H} 2 \mathrm{O}$ & $\mathrm{MeOH}$ & & AmFo, FoA & 2019 & {$[48]$} \\
\hline & C18 BEH & $\mathrm{MeOH}$ & 2:5 ACN:IPA & & AmAc, FoA & 2019 & {$[54]$} \\
\hline & C18 HSS & N/A & $\mathrm{N} / \mathrm{A}$ & & $\mathrm{N} / \mathrm{A}$ & 2019 & [61] \\
\hline
\end{tabular}

*nano-LC, **APCl instead of ESI, ***narrow-bore UHPLC

BEH: ethylene bridged hybrid, CSH: charged surface hybrid, HSS: high strength silica, SB: stable bond packing 


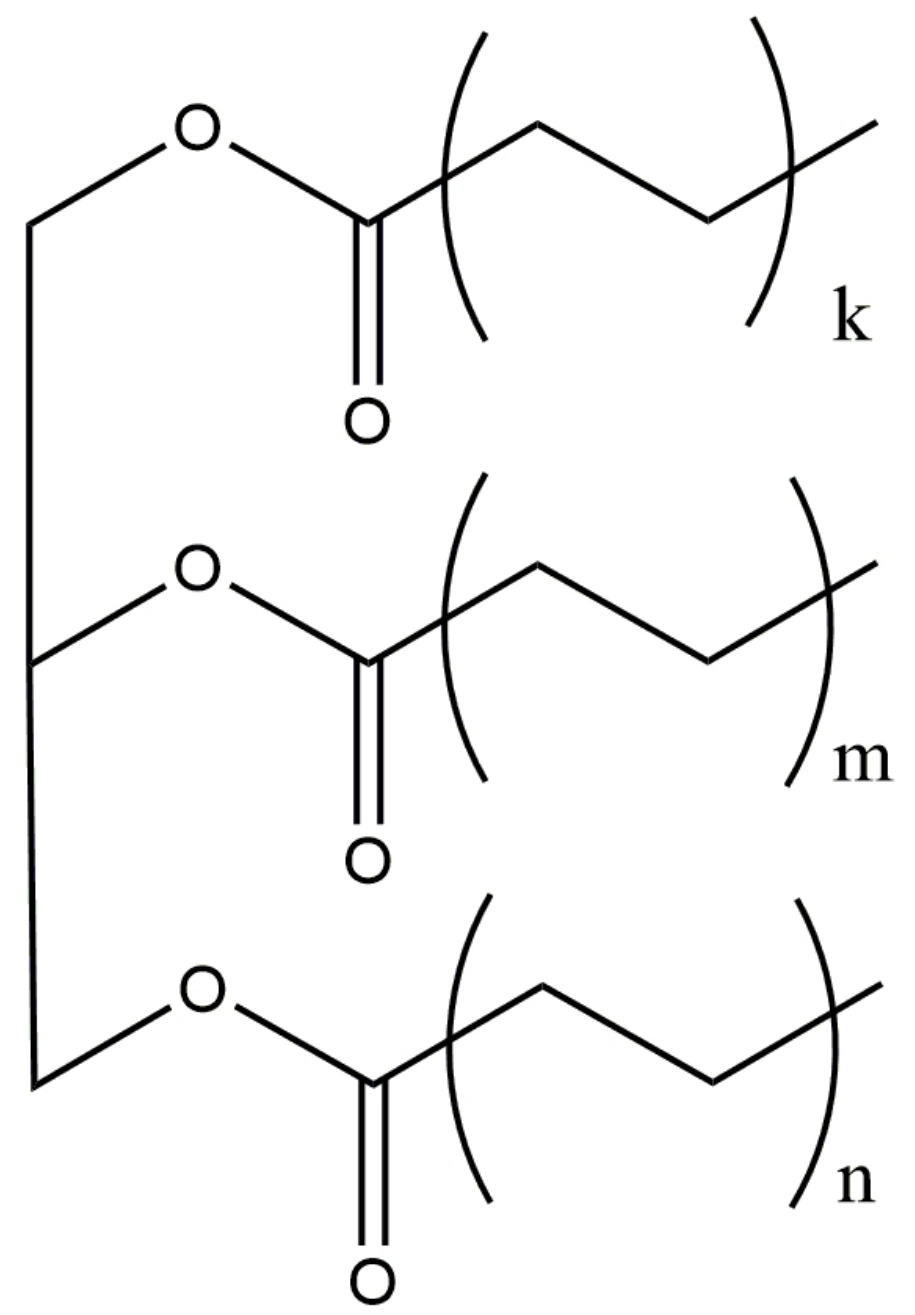

$\mathrm{TG}(\mathrm{N}[\mathrm{k}+2]: 0, \mathrm{~N}[\mathrm{~m}+2]: 0, \mathrm{~N}[\mathrm{n}+2]: 0)$

$\mathrm{k}=\mathrm{m}=\mathrm{n}=16 \Rightarrow \mathrm{TG}(18: 0,18: 0,18: 0), \mathrm{TG}(54: 0)$

$\mathrm{k}+\mathrm{m}+\mathrm{n}=54=>\mathrm{TG}(54: 0)$ (FAs unknown) 


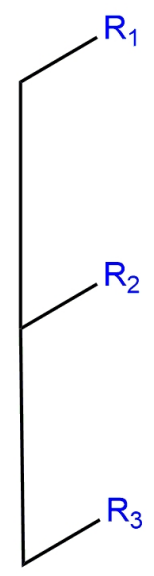

MG: $\mathrm{R}_{1}=\mathrm{FA}, \mathrm{R}_{2}=\mathrm{OH}, \mathrm{R}_{3}=\mathrm{OH}$ or $\mathrm{R}_{1}=\mathrm{OH}, \mathrm{R}_{2}=\mathrm{FA}, \mathrm{R}_{3}=\mathrm{OH}$

DG: $\mathrm{R}_{1}=\mathrm{FA}, \mathrm{R}_{2}=\mathrm{FA}, \mathrm{R}_{3}=\mathrm{OH}$ or $\mathrm{R}_{1}=\mathrm{FA}, \mathrm{R}_{2}=\mathrm{OH}, \mathrm{R}_{3}=\mathrm{FA}$

TG: $\mathrm{R}_{1}=\mathrm{FA}, \mathrm{R}_{2}=\mathrm{FA}, \mathrm{R}_{3}=\mathrm{FA}$

PC: e.g. $\mathrm{R}_{1}=\mathrm{OPO}_{3}{ }^{-} \mathrm{CH}_{2} \mathrm{CH}_{2} \mathrm{~N}\left(\mathrm{CH}_{3}\right)_{3}{ }^{+}, \mathrm{R}_{2}=\mathrm{FA}, \mathrm{R}_{3}=\mathrm{FA}$

PE: e.g. $\mathrm{R}_{1}=\mathrm{OPO}_{3}{ }^{-} \mathrm{CH}_{2} \mathrm{CH}_{2} \mathrm{NH}_{3}{ }^{+}, \mathrm{R}_{2}=\mathrm{FA}, \mathrm{R}_{3}=\mathrm{FA}$

PG: e.g. $\mathrm{R}_{1}=\mathrm{OPO}_{3}{ }^{-} \mathrm{CH}_{2} \mathrm{CH}(\mathrm{OH}) \mathrm{CH}_{2} \mathrm{OH}, \mathrm{R}_{2}=\mathrm{FA}, \mathrm{R}_{3}=\mathrm{FA}$

PI: e.g. $\mathrm{R}_{1}=\mathrm{OPO}_{3}{ }^{-} \mathrm{CH}_{2} \mathrm{CH}_{2} \mathrm{NH}_{3}{ }^{+}, \mathrm{R}_{2}=\mathrm{FA}, \mathrm{R}_{3}=\mathrm{FA}$

PS: e.g. $\mathrm{R}_{1}=\mathrm{OPO}_{3}{ }^{-} \mathrm{CH}_{2} \mathrm{CH}\left(\mathrm{COO}^{-}\right) \mathrm{NH}_{2}, \mathrm{R}_{2}=\mathrm{FA}, \mathrm{R}_{3}=\mathrm{FA}$

LPC: e.g. $\mathrm{R}_{1}=\mathrm{OPO}_{3}{ }^{-} \mathrm{CH}_{2} \mathrm{CH}_{2} \mathrm{~N}\left(\mathrm{CH}_{3}\right)_{3}{ }^{+}, \mathrm{R}_{2}=\mathrm{FA}, \mathrm{R}_{3}=\mathrm{OH}$

LPE: e.g. $\mathrm{R}_{1}=\mathrm{OPO}_{3} \mathrm{CH}_{2} \mathrm{CH}_{2} \mathrm{NH}_{3}{ }^{+}, \mathrm{R}_{2}=\mathrm{FA}, \mathrm{R}_{3}=\mathrm{OH}$

FA: fatty acid residue

B

PC(...):

PC(O-...):

PC(P-...):
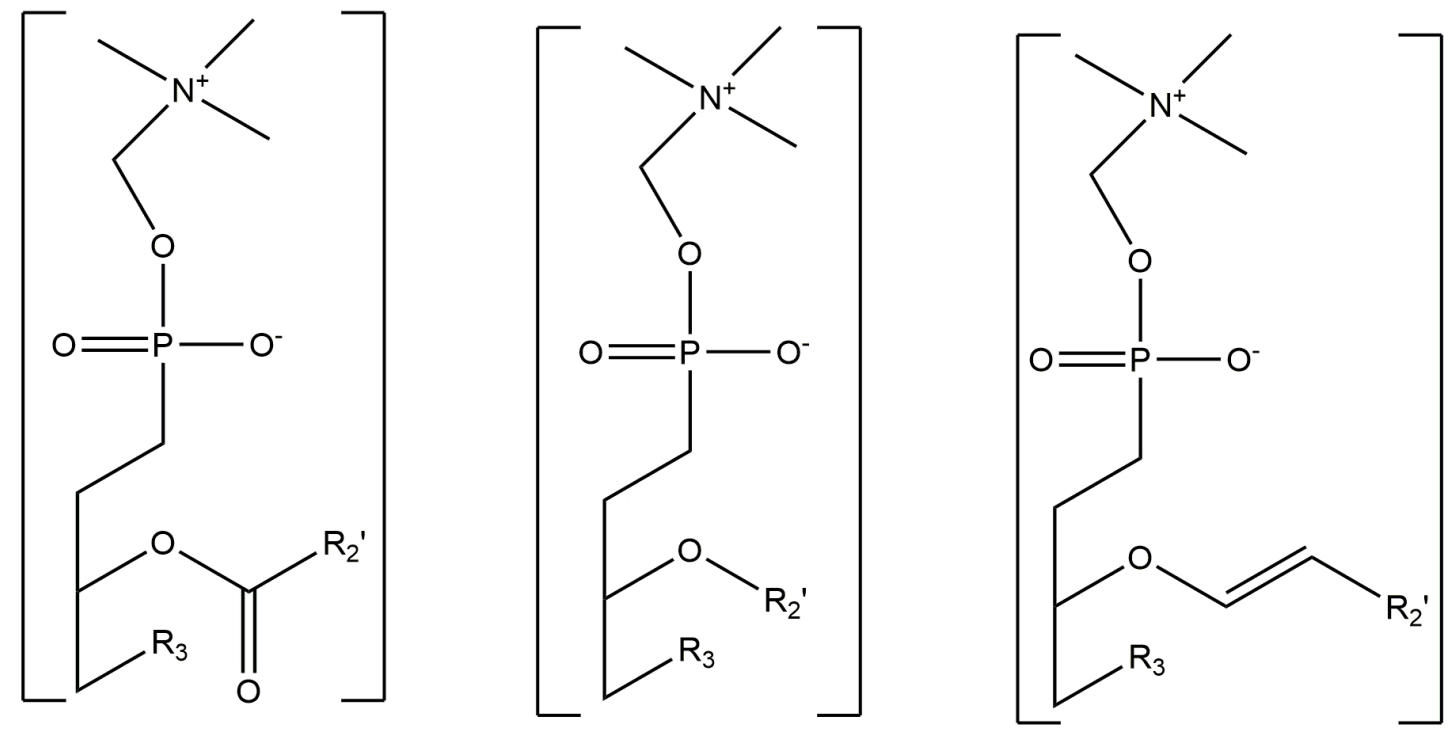


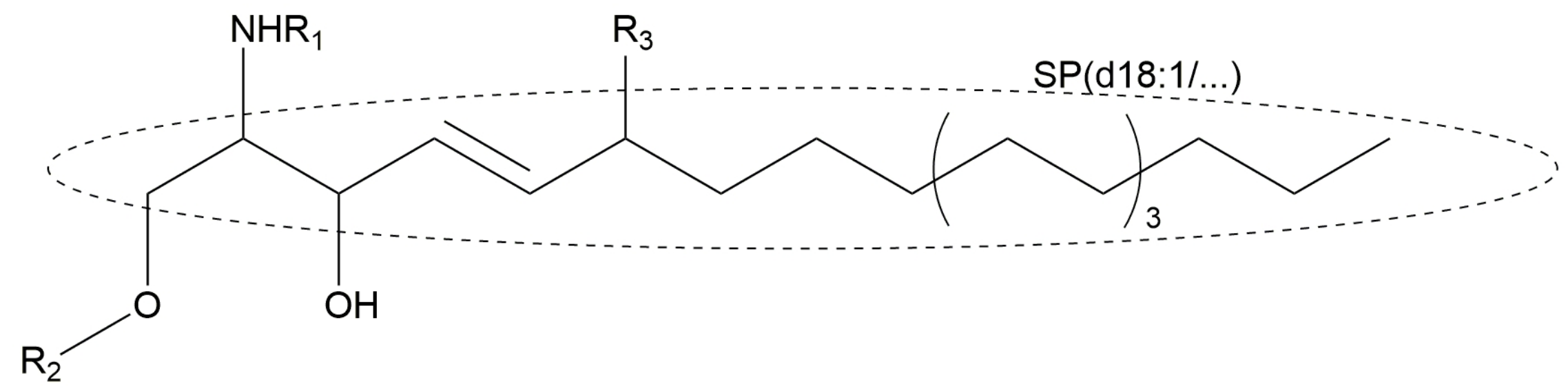

$$
\begin{aligned}
& \text { Cer: } \quad \mathrm{R}_{1}=\mathrm{CO}-\mathrm{FA}, \mathrm{R}_{2}=\mathrm{H}, \mathrm{R}_{3}=\mathrm{H} \\
& \text { S1P: } \quad \mathrm{R}_{1}=\mathrm{H}, \mathrm{R}_{2}=\mathrm{PO}_{3}{ }^{-} \mathrm{H}, \mathrm{R}_{3}=\mathrm{H} \\
& \text { SA1P*: } \quad \mathrm{R}_{1}=\mathrm{H}, \mathrm{R}_{2}=\mathrm{PO}_{3}{ }^{-} \mathrm{H}, \mathrm{R}_{3}=\mathrm{H} \\
& \text { SM(Cer-PC): } \quad \mathrm{R}_{1}=\mathrm{CO}-\mathrm{FA}, \mathrm{R}_{2}=\mathrm{PO}_{3}{ }^{-} \mathrm{CH}_{2} \mathrm{CH}_{2} \mathrm{NH}_{2}, \mathrm{R}_{3}=\mathrm{H} \\
& \text { SM(Cer-PE): } \quad \mathrm{R}_{1}=\mathrm{CO}-\mathrm{FA}, \mathrm{R}_{2}=\mathrm{PO}_{3}{ }^{-} \mathrm{CH}_{2} \mathrm{CH}_{2} \mathrm{~N}\left(\mathrm{CH}_{3}\right)_{3}{ }^{+}, \mathrm{R}_{3}=\mathrm{H} \\
& \text { Phytosphingosine*: } \quad \mathrm{R}_{1}=\mathrm{H}, \mathrm{R}_{2}=\mathrm{H}, \mathrm{R}_{3}=\mathrm{OH}
\end{aligned}
$$$$
\text { 6-hydroxy-sphingosine: } \mathrm{R}_{1}=\mathrm{H}, \mathrm{R}_{2}=\mathrm{H}, \mathrm{R}_{3}=\mathrm{OH}
$$

S1P: sphingosine phosphate

SA1P: sphinganine phosphate

$$
\text { *no double bond }
$$


(a)

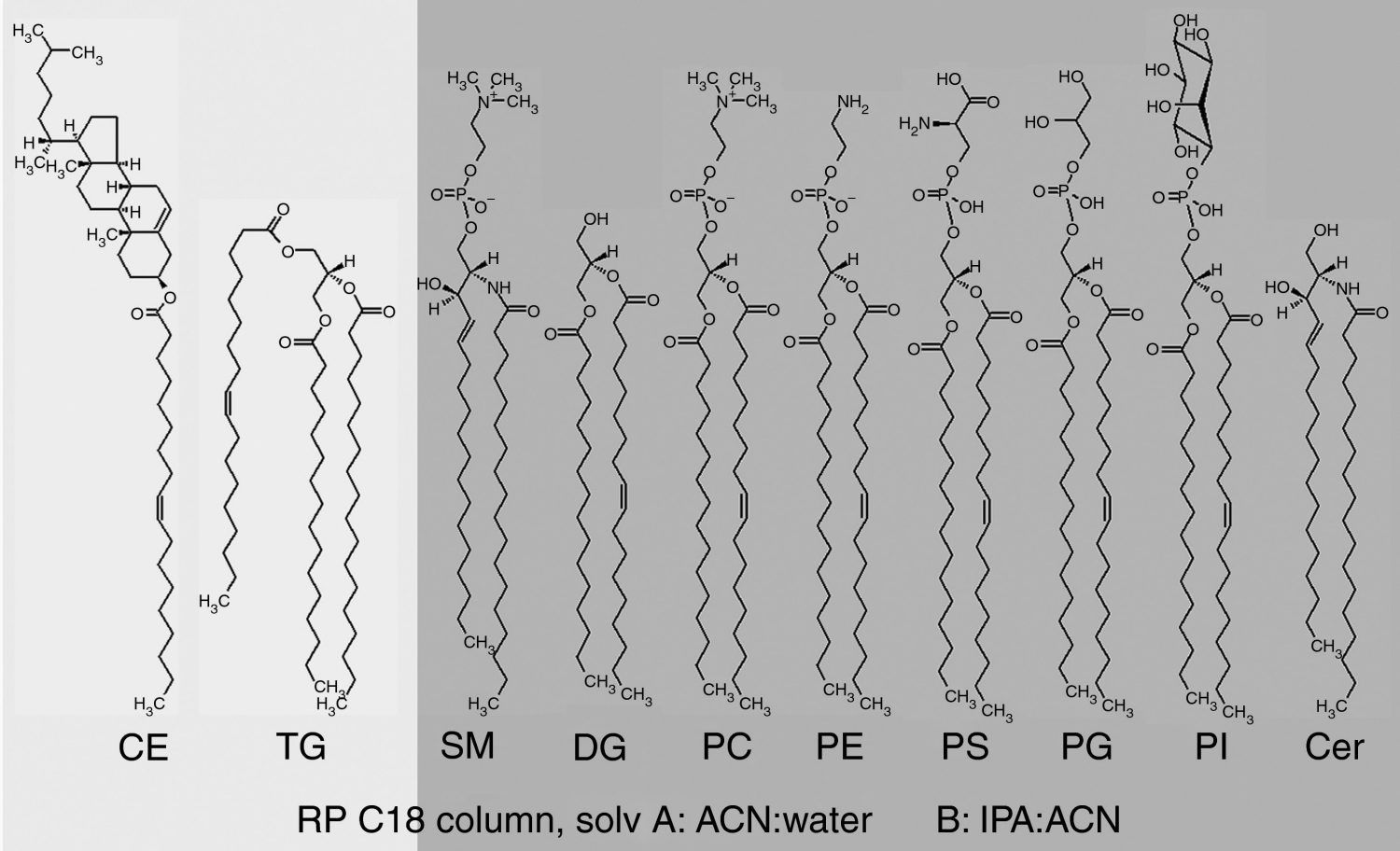

NP Silica, solv A\&B IPA:Hexane

(b)

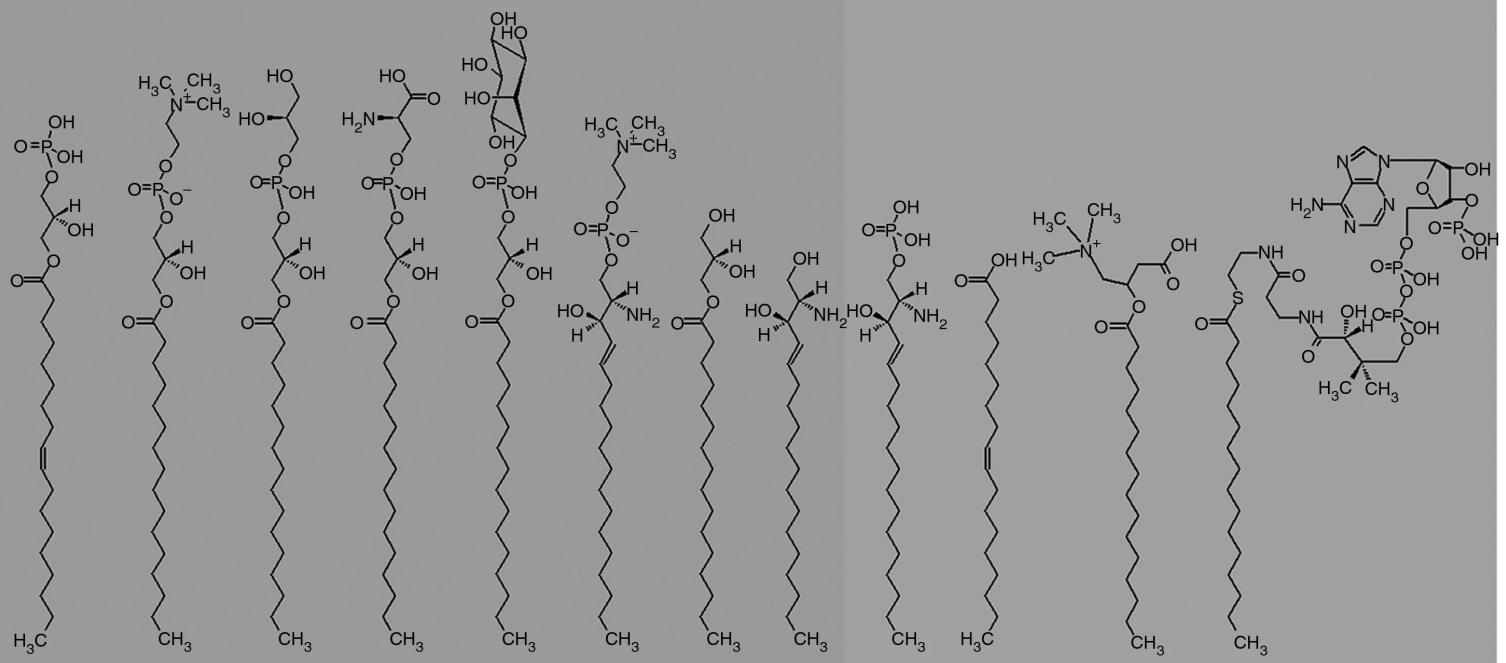

LPA LPC LPG LPS LPI LSM MG So S1P FA AcyICN AcylCoA

RP C18 column, solv A: water $\left(0.05 \% \mathrm{NH}_{4} \mathrm{OH}\right) \quad \mathrm{B}$ : $\mathrm{MeOH}\left(0.05 \% \mathrm{NH}_{4} \mathrm{OH}\right)$

HILIC column,

A: $0.2 \%$ formic acid, $200 \mathrm{mM} \mathrm{NH}_{4} \mathrm{HCOO}$ in water

B: $0.2 \%$ formic acid in ACN 


\section{Tables:}

Table 1. Instrumental approaches and MS or MS/MS chromatographic methods. Ionization mode was done either in positive (pos), negative (neg) or polarity switch (Switch) ion mode.

\begin{tabular}{|c|c|c|c|c|c|c|}
\hline Approach & Column & ESI mode & MS/MS & Tandem mode & Year & Citation \\
\hline UHPLC-ESI-Orbitrap & & Pos/Neg & No & - & 2017 & {$[2]$} \\
\hline UHPLC-ESI-QOrbitrap & Silica & Pos/Neg & No & - & 2017 & [3] \\
\hline UHPLC-ESI-QTOF & $\mathrm{CSH}$ & Pos & No & - & 2017 & {$[4]$} \\
\hline UHPLC-HESI-Orbitrap & $\mathrm{BEH}$ & Switch & No & - & 2017 & {$[5]$} \\
\hline UHPLC-Zspray-IMS-QTOF & $\mathrm{CSH}$ & Pos/Neg & No & - & 2017 & [6] \\
\hline UHPLC-ESI-QqQ-MS/MS & & $\begin{array}{l}\text { Pos (SPs)/Neg } \\
\text { (SM) }\end{array}$ & Yes & MRM & 2017 & [7] \\
\hline UHPLC-ESI-QqQ-MS/MS & Silica & Pos/Neg & Yes & MRM & 2017 & [8] \\
\hline UHPLC-ESI-QOrbitrap-MS/MS & $\mathrm{C} 8 \mathrm{BEH}$ & Pos/Neg & Yes & MSE & 2017 & [9] \\
\hline UHPLC-ESI-QTOF-MS/MS & $\mathrm{BEH}$ & Pos/Neg & Yes & MSE & 2017 & [10] \\
\hline UHPLC-ESI-QTOF-MS/MS & $\mathrm{CSH}$ & Pos/Neg & Yes & MSE & 2017 & {$[11]$} \\
\hline UHPLC-ESI-QTOF-MS/MS & HSS & Pos/Neg & Yes & MSE & 2017 & [12] \\
\hline UHPLC-ESI-QTOF-MS/MS & & Pos & Yes & MSE & 2017 & [13] \\
\hline UHPLC-ESI-QTOF-MSMS & $\mathrm{CSH}$ & Pos/Neg & Yes & MSE & 2017 & [14] \\
\hline UHPLC-ESI-QTOF-MS/MS & HSS & Pos/Neg & Yes & MSE, PRM & 2017 & [15] \\
\hline UHPLC-ESI-IMS-QTOF-MS/MS & & Pos & Yes & $N / A$ & 2017 & [16] \\
\hline UHPLC-ESI-QOrbitrap-MS/MS & $\mathrm{BEH}$ & Switch & Yes & $N / A$ & 2017 & [17] \\
\hline UHPLC-ESI-QOrbitrap-MS/MS & & Pos/Neg & Yes & $N / A$ & 2017 & [18] \\
\hline UHPLC-HESI-QOrbitrap-MS/MS & $\mathrm{BEH}$ & Pos/Neg & Yes & PIS & 2017 & [19] \\
\hline UHPLC-HESI-QOrbitrap-MS/MS & & Pos/Neg & Yes & PIS & 2017 & [20] \\
\hline UHPLC-ESI-QTOF-MS/MS & & Pos/Neg & Yes & PIS? & 2017 & {$[21]$} \\
\hline UHPLC-HESI-QOrbitrap-MS/MS & $\mathrm{CSH}$ & Pos/Neg & Yes & product ion scan & 2017 & [22] \\
\hline UHPLC-ESI(/APCI)-QqQ-MS/MS & $* *$ & Switch & Yes & SIM & 2017 & [23] \\
\hline AF4, nUHPLC-ESI-QqQ-MS/MS & $*$ & Pos/Neg & Yes & SRM, PIS & 2017 & [24] \\
\hline UHPLC-ESI-IMS-QTOF & $\mathrm{CSH}$ & Pos & No & - & 2018 & [25] \\
\hline UHPLC-ESI-QOrbitrap & Silica & Pos/Neg & No & - & 2018 & {$[26]$} \\
\hline UHPLC-HESI-QOrbitrap & HSS & Switch & No & - & 2018 & {$[27]$} \\
\hline UHPLC-ESI-QTOF & BEH & Pos & No? & - & 2018 & [28] \\
\hline UHPLC-ESI-Qtrap-MS/MS & SB & Neg & Yes & $\begin{array}{l}\text { enh. product ion } \\
\text { scan, MRM }\end{array}$ & 2018 & [29] \\
\hline UHPLC-ESI-QQQ-MS/MS & C8 BEH & Pos/Neg & Yes & MRM & 2018 & [30] \\
\hline UHPLC-HESI-QOrbitrap-MS/MS & $\begin{array}{l}\text { BEH, } \\
\text { CSH, } \\
\text { Amide }\end{array}$ & Pos/Neg & Yes & MRM & 2018 & [31] \\
\hline UHPLC-ESI-QTOF-MS/MS & $\mathrm{BEH}$ & Pos/Neg & Yes & MSE & 2018 & [32] \\
\hline
\end{tabular}

Enh.: enhanced, PIS: precursor ion scan, *nano-LC, **APCI instead of ESI, ***narrow-bore LC

BEH: ethylene bridged hybrid, CSH: charged surface hybrid, HSS: high strength silica,

SB: stable bond packing 


\begin{tabular}{|c|c|c|c|c|c|c|}
\hline Approach & Column & ESI mode & MS/MS & Tandem mode & Year & Citation \\
\hline UHPLC-ESI-QTOF-MS/MS & & Pos/Neg & Yes & MSE & 2018 & [33] \\
\hline UHPLC-ESI-QOrbitrap-MS/MS & $\mathrm{CSH}$ & Pos/Neg & Yes & MSE, MRM & 2018 & [34] \\
\hline UHPLC-HESI-QOrbitrap & & Pos/Neg & Yes & $\mathrm{N} / \mathrm{A}$ & 2018 & [35] \\
\hline UHPLC-ESI-QTOF-MS/MS & & Pos/Neg & Yes & PIS & 2018 & [36] \\
\hline UHPLC-QTOF-MS/MS & $\mathrm{BEH}$ & Pos/Neg & Yes & PIS & 2018 & [37] \\
\hline UHPLC-ESI-QTOF-MS/MS & & Pos/Neg & Yes & Targeted & 2018 & [38] \\
\hline UHPLC-QTOF-MS/MS & $\begin{array}{l}\mathrm{CSH} \\
\mathrm{HSS}\end{array}$ & Pos/Neg & Yes & Targeted & 2018 & [39] \\
\hline UHPLC-ESI-QTOF-MS/MS & $\mathrm{BEH}$ & Pos/Neg & Yes & product ion scan & 2018 & [40] \\
\hline AF4, nUHPLC-ESI-QqQ-MS/MS & $*$ & Switch & Yes & SRM & 2018 & [41] \\
\hline AF4, nUHPLC-ESI-QqQ-MS/MS & $*$ & Pos/Neg & Yes & SRM & 2018 & [42] \\
\hline UHPLC-ESI-QQQ & & Neg & Yes & SRM & 2018 & [43] \\
\hline $\begin{array}{l}\text { UHPLC-ESI(neg: APCI)-QTOF- } \\
\text { MS/MS }\end{array}$ & $* *$ & Pos/Neg & Yes & SWATH, PRM & 2018 & [44] \\
\hline nUHPLC-NSI-QTOF-MS/MS & $* / * * *$ & Pos/Neg & Yes & Top10 ddMS2, PRM & 2018 & [45] \\
\hline UHPLC-HESI-QOrbitrap-MS/MS & $\mathrm{BEH}$ & Pos/Neg & Yes & top10-ddMS2 & 2018 & [46] \\
\hline UHPLC-APCI-QLIT-MS & HILIC** & Pos & No & - & 2019 & [47] \\
\hline UHPLC-ESI-TOF & C8 BEH & Pos & No & - & 2019 & [48] \\
\hline UHPLC-HESI-QOrbitrap & Phenyl & Switch & No & - & 2019 & [49] \\
\hline UHPLC-ESI-Orbitrap-MS/MS & & Pos/Neg & Yes & autoMS & 2019 & [50] \\
\hline UHPLC-HESI-QOrbitrap-MS/MS & F5 & Pos/Neg & Yes & autoMs & 2019 & [51] \\
\hline UHPLC-ESI-Qtrap-MS/MS & & Pos/Neg & Yes & MRM & 2019 & [52] \\
\hline UHPLC-ESI-QTOF-MS/MS & & Pos/Neg & Yes & $\begin{array}{l}\text { MRM (GPs), } \\
\text { product ion scan } \\
\text { (FAs) }\end{array}$ & 2019 & [53] \\
\hline UHPLC-ESI-QTOF-MS/MS & $\mathrm{BEH}$ & Pos & Yes & MRM, PIS & 2019 & [54] \\
\hline UHPLC-ESI-QOrbitrap-MS/MS & $\mathrm{CSH}$ & Pos/Neg & Yes & MSE & 2019 & [55] \\
\hline UHPLC-ESI-QOrbitrap-MS/MS & $\mathrm{C} 8$ & Pos/Neg & Yes & MSE & 2019 & [56] \\
\hline UHPLC-ESI-QOrbitrap-MS/MS & & Pos/Neg & Yes & MSE & 2019 & [57] \\
\hline UHPLC-ESI-QOrbitrap-MS/MS & $\mathrm{CSH}$ & Pos/Neg & Yes & MSE & 2019 & [58] \\
\hline UHPLC-ESI-QTOF-MS/MS & & Pos/Neg & Yes & MSE & 2019 & [59] \\
\hline UHPLC-ESI-QTOF-MS/MS & & Pos/Neg & Yes & MSE & 2019 & [60] \\
\hline UHPLC-ESI-QTOF-MS/MS & HSS & Pos & Yes & MSE & 2019 & [61] \\
\hline UHPLC-ESI-QOrbitrap-MS/MS & $\mathrm{CSH}$ & Pos/Neg & Yes & Untargeted & 2019 & {$[62]$} \\
\hline UHPLC-ESI-QOrbitrap-MS/MS & $\mathrm{BEH}$ & Pos/Neg & Yes & $\mathrm{N} / \mathrm{A}$ & 2019 & [63] \\
\hline UHPLC-ESI-QOrbitrap-MS/MS & $\mathrm{CSH}^{* *}$ & Pos/Neg & Yes & $\mathrm{N} / \mathrm{A}$ & 2020 & [64] \\
\hline UHPLC-ESI-QOrbitrap-MS/MS & $\mathrm{CSH}$ & Pos/Neg & Yes & PIS & 2019 & [65] \\
\hline
\end{tabular}

Enh.: enhanced, PIS: precursor ion scan, *nano-LC, **APCI instead of ESI, ***narrow-bore LC

BEH: ethylene bridged hybrid, CSH: charged surface hybrid, HSS: high strength silica,

SB: stable bond packing 


\begin{tabular}{|c|c|c|c|c|c|c|}
\hline Approach & Column & ESI mode & MS/MS & Tandem mode & Year & Citation \\
\hline UHPLC-ESI-QTOF-MS/MS & $\mathrm{CSH}$ & Pos/Neg & Yes & PIS & 2019 & [66] \\
\hline UHPLC-ESI-QOrbitrap-MS/MS & $\begin{array}{l}\mathrm{CSH} \\
\mathrm{HSS}\end{array}$ & Pos & Yes & Targeted & 2019 & {$[60]$} \\
\hline UHPLC-HESI-QOrbitrap-MS/MS & HILIC** & Neg & Yes & Targeted & 2019 & [47] \\
\hline UHPLC-ESI-QTOF-MS/MS & $\mathrm{BEH}^{* *}$ & Pos/Neg & Yes & $\begin{array}{l}\text { Product ion scan, } \\
\text { NLS }\end{array}$ & 2019 & {$[67]$} \\
\hline UHPLC-ESI-IMS-QTOF-MS/MS & $\mathrm{CSH}$ & Neg & Yes & $\begin{array}{l}\text { Product ion scan, } \\
\text { PIS }\end{array}$ & 2019 & [68] \\
\hline UHPLC-ESI-QTOF-MS/MS & $\mathrm{CSH}$ & Pos/Neg & Yes & SONAR & 2019 & [69] \\
\hline nUHPLC-ESI-QTOF-MS/MS & & Pos/Neg & Yes & SRM & 2019 & [70] \\
\hline nUHPLC-NSI-QTOF-MS/MS & $*$ & Pos/Neg & Yes & SRM & 2019 & [71] \\
\hline AF4, nUHPLC-ESI-QOrbitrap-MS/MS & & Pos/Neg & Yes & SRM, PIS & 2019 & {$[72]$} \\
\hline UHPLC-ESI-QTOF-MS/MS & & Pos/Neg & Yes & SWATH & 2019 & [73] \\
\hline UHPLC-ESI-QTOF-MS/MS & $\mathrm{CSH}$ & Pos/Neg & Yes & SWATH & 2019 & [74] \\
\hline UHPLC-ESI-QTOF-MS/MS & $\mathrm{CSH}$ & Pos/Neg & Yes & SWATH & 2019 & [75] \\
\hline UHPLC-ESI-QTOF-MS/MS & $\mathrm{CSH}$ & Pos/Neg & Yes & SWATH & 2019 & [76] \\
\hline UHPLC-HESI-QOrbitrap-MS/MS & $\mathrm{CSH}$ & Pos/Neg & Yes & Top10-ddMS2 & 2019 & [77] \\
\hline UHPLC-ESI-QTOF-MS/MS & & Pos/Neg & Yes & N/A & 2019 & [78] \\
\hline UHPLC-HESI-QOrbitrap-MS/MS & $\mathrm{CSH}$ & Pos/Neg & Yes & Targeted & 2019 & [79] \\
\hline
\end{tabular}

Enh.: enhanced, PIS: precursor ion scan, *nano-LC, **APCI instead of ESI, ***narrow-bore LC

BEH: ethylene bridged hybrid, CSH: charged surface hybrid, HSS: high strength silica,

SB: stable bond packing 
Table 2. New analytic method developments and application enhancements in lipidomics

\begin{tabular}{|c|c|c|}
\hline Year & Citation & Subtheme \\
\hline 2017 & [21] & Improvement to in-silico fragmentation prediction \\
\hline 2017 & [9] & Ultrahigh performance chromatography lipidomics \\
\hline 2017 & [14] & Super absorbent polymer extraction chip testing \\
\hline 2017 & [95] & Single-cell resolution, PDMS microfluid droplet chip Raman method \\
\hline 2017 & [96] & LipidMatch comparison to other software \\
\hline 2017 & {$[23]$} & Method development, low resolution MS identification \\
\hline 2017 & [19] & in vitro computational data analysis method, excessive adipocyte lipolysis \\
\hline 2017 & [16] & Comparison of LC/MS, SCF/MS and DIMS, kidney cancer patient analysis \\
\hline 2017 & {$[22]$} & Bee pollen analysis, method validation \\
\hline 2017 & {$[20]$} & Lipid extraction comparison with pancreatic cancer cell line \\
\hline 2017 & [17] & Data processing improvements, nonalcoholic fatty liver disease analysis \\
\hline 2018 & {$[25]$} & Machine learning algorithms for CCS values \\
\hline 2018 & {$[45]$} & Reproducible nano-LC NSI method \\
\hline 2018 & [27] & Multi-matrix platform validation \\
\hline 2018 & {$[46]$} & Optimization of established extraction techniques \\
\hline 2018 & {$[30]$} & Multimatrix method development, a mixture of untargeted/targeted \\
\hline 2019 & {$[66]$} & $\begin{array}{l}\text { Extraction comparison ("IPA-75", "IPA-90" vs "Bligh \& Dyer"), MS-DIAL, } \\
\text { SWATH }\end{array}$ \\
\hline 2019 & {$[68]$} & Oxylipin, eicosanoid and FA identification method with IMS-MS/MS (DIA), \\
\hline 2019 & {$[76]$} & $\begin{array}{l}\text { Guide for choosing a suitable strategy for an ISTD-based untargeted } \\
\text { approach }\end{array}$ \\
\hline 2019 & [51] & Method for hepatotoxicity evaluation \\
\hline 2019 & [69] & $\begin{array}{l}\text { DIA method with rapid "SONAR" sequential ion feeding scan, } \\
\text { sitaxentan (antihypertension drug) effects in animals }\end{array}$ \\
\hline
\end{tabular}


Table 3. Mass-to-charge values for several lipid classes found during research. Ch: cholesterol and its derivatives, Hex: SPs hexosyl-derivatives

\begin{tabular}{|c|c|c|c|c|c|c|c|c|c|c|c|c|c|c|c|c|c|c|c|c|}
\hline Citation & Table & FA & FFA & GL & GP & LPL & PL & Ox & $\begin{array}{l}\text { Sulfo- } \\
\text { GP }\end{array}$ & SP & Hex & ST & $\mathrm{Ch}$ & SL & PK & PR & adduct & $\mathrm{m} / \mathrm{z}$ & CE & more \\
\hline [120] & S3 & $x$ & $x$ & $x$ & $x$ & $x$ & $x$ & & & $x$ & $x$ & & $x$ & & & & $\mathrm{x}$ & $x$ & & \\
\hline [121] & A2 & $x$ & $x$ & $x$ & $x$ & $x$ & & & & $x$ & & & & & & & $x$ & $x$ & & Metabolomic study \\
\hline [5] & S1 & $x$ & $x$ & $x$ & $x$ & & $x$ & & & $x$ & & $x$ & $\mathrm{x}$ & $x$ & $x$ & $x$ & $x$ & $x$ & & Authentic standards \\
\hline [49] & S7 & $x$ & $x$ & $x$ & $x$ & & & & & & & $x$ & $x$ & & & $x$ & & $x$ & & Metabolomic study \\
\hline [7] & S1, S2 & $x$ & $x$ & & $x$ & $x$ & $x$ & & $x$ & $x$ & & $x$ & & $x$ & & $x$ & $x$ & $x$ & & Metabolomic study \\
\hline [39] & S6 & $x$ & $x$ & & $x$ & $x$ & & & & & & & & $x$ & & & $x$ & $x$ & & Protonated ions only \\
\hline [122] & S1, S4 & $x$ & $x$ & & $x$ & $x$ & & & & & & & & & & & $x$ & $x$ & & Daphnia Magna baseline \\
\hline [123] & S1 & $x$ & & $x$ & $x$ & $x$ & & & & $x$ & $x$ & & & & & & & $x$ & & FA-chains, lipoproteins \\
\hline [63] & S2, S3 & $x$ & & & $x$ & $x$ & $x$ & $x$ & & & & & & & & & $x$ & $x$ & & Metabolomic study \\
\hline [43] & T2 & $x$ & & & & & & & & & & & & & & & & $x$ & $x$ & Prostaglandines \\
\hline [23] & $\begin{array}{c}\text { S1, S2, } \\
\text { S3 }\end{array}$ & & $x$ & $x$ & $x$ & $x$ & $x$ & & $x$ & $x$ & & & $x$ & $x$ & & & $x$ & $x$ & & \\
\hline [30] & $\mathrm{T} 1, \mathrm{~T} 2$ & & $x$ & $x$ & $x$ & $x$ & $x$ & & & $x$ & $x$ & & & & & & $x$ & $x$ & & part of the precursors \\
\hline [86] & S1 & & $x$ & $x$ & $x$ & $x$ & $x$ & & & $x$ & & $x$ & & & $x$ & & $x$ & $x$ & & Column comparison \\
\hline [124] & S1 & & $x$ & $x$ & $x$ & $x$ & $x$ & & & $x$ & & & $x$ & & & & $x$ & $x$ & & \\
\hline$[75]$ & S1 & & $x$ & $x$ & $x$ & $x$ & & & & $x$ & & & $x$ & & & & $x$ & $x$ & & Standards, SWATH \\
\hline [22] & $\mathrm{T} 2, \mathrm{~T} 3$ & & $x$ & $x$ & $x$ & $x$ & & & & $x$ & & & & & & & $x$ & $x$ & & Gene expression manipulation \\
\hline [76] & S1 & & $x$ & $x$ & $x$ & $x$ & & & & & & & & & & & $x$ & & & \\
\hline$[66]$ & S1 & & $x$ & $x$ & $x$ & & $x$ & & & $x$ & & & & $x$ & & & $x$ & $x$ & & \\
\hline [73] & $\begin{array}{c}\text { A1, A5, } \\
\text { A10 }\end{array}$ & & $x$ & & $x$ & $x$ & & & & & & & & & & & $x$ & $x$ & $x$ & SWATH, exclusion list MS-DIAL \\
\hline [37] & $\mathrm{T} 1$ & & $x$ & & & $x$ & $x$ & & & & & & & & & & & $x$ & & PAF C-16 \\
\hline
\end{tabular}




\begin{tabular}{|c|c|c|c|c|c|c|c|c|c|c|c|c|c|c|c|c|c|c|c|c|}
\hline Citation & Table & FA & FFA & GL & GP & LPL & PL & Ox & $\begin{array}{c}\text { Sulfo- } \\
\text { GP }\end{array}$ & SP & Hex & ST & $\mathrm{Ch}$ & SL & PK & PR & adduct & $\mathrm{m} / \mathrm{z}$ & CE & more \\
\hline [19] & S2 & & $x$ & & & & & & & & & & & & & & & $x$ & & \\
\hline$[42]$ & S3 & & & $x$ & $x$ & $x$ & $X$ & & $x$ & $x$ & $x$ & & & & & & & $x$ & & \\
\hline$[41]$ & $\mathrm{S} 1, \mathrm{~S} 2$ & & & $x$ & $x$ & $x$ & $X$ & & & $x$ & $x$ & & & & & & $x$ & $x$ & & List for possible acyl chains (S3) \\
\hline$[8]$ & $\mathrm{T} 2$ & & & $x$ & $x$ & $x$ & $X$ & & & $x$ & $x$ & & & & & & & $x$ & & Abundances in 5 cancer types \\
\hline [9] & S7 & & & $x$ & $x$ & $x$ & $X$ & & & $x$ & $x$ & & & & & & $x$ & $x$ & & Adduct list \\
\hline [14] & $\mathrm{S} 1, \mathrm{~S} 2$ & & & $x$ & $x$ & $x$ & $X$ & & & $x$ & & & $x$ & $x$ & & & $x$ & $x$ & & $\mathrm{APCl}$ \\
\hline [16] & $\mathrm{S} 2$ & & & $x$ & $x$ & $x$ & $x$ & & & $x$ & & & $x$ & & & & $x$ & $x$ & & $\begin{array}{l}\text { Adduct list, UHPLC/SFC/DIMS \& tissue } \\
\text { comparison, Des-lipids }\end{array}$ \\
\hline [60] & S1 & & & $x$ & $x$ & $x$ & $X$ & & & $x$ & & & $x$ & $x$ & & & & $x$ & & \\
\hline [48] & S3 & & & $x$ & $x$ & $x$ & $x$ & & & $x$ & & & $x$ & & & & & $x$ & & \\
\hline [11] & S2, S3 & & & $x$ & $x$ & $x$ & $X$ & & & $x$ & & & & & & & $x$ & $x$ & & \\
\hline [65] & $\begin{array}{l}\text { S1, S2, } \\
\text { S3 }\end{array}$ & & & $x$ & $x$ & $x$ & $x$ & & & $x$ & & & & & & & $x$ & $x$ & & $\mathrm{PE}(16: 0 / 18: 1)+\mathrm{H} \mathrm{m} / \mathrm{z}$ fragments \\
\hline$[96]$ & S1, S4 & & & $x$ & $x$ & $x$ & & $x$ & & & & & $x$ & $x$ & & & $x$ & $x$ & & Adduct list, Software comparison \\
\hline$[34]$ & $\mathrm{S} 1, \mathrm{~S} 2$ & & & $x$ & $x$ & $x$ & & & & $x$ & & & $x$ & & & & $x$ & $x$ & $x$ & MRM validation, methylated species \\
\hline$[25]$ & $\mathrm{S} 1, \mathrm{~S} 2$ & & & $x$ & $x$ & $x$ & & & & $x$ & & & $x$ & & & & $x$ & & & CCS \\
\hline$[52]$ & A3 & & & $x$ & $x$ & $x$ & & & & $x$ & & & $x$ & & & & & & & SWATH, standards only \\
\hline [15] & T1 & & & $x$ & $x$ & $x$ & & & & $x$ & & & & $x$ & & & & $x$ & & Saccharolipids \\
\hline [45] & $\begin{array}{l}\text { S4, } 56 \\
\text { S8 }\end{array}$ & & & $x$ & $x$ & $x$ & & & & $x$ & & & & $x$ & & & $x$ & $x$ & $x$ & PRM, sensitivity data \\
\hline [72] & S1 & & & $x$ & $x$ & $x$ & & & & $x$ & & & & & & & $x$ & $x$ & $x$ & \\
\hline [79] & $\begin{array}{l}\text { S2, S3, } \\
\text { S4 }\end{array}$ & & & $x$ & $x$ & $x$ & & & & $x$ & & & & & & & $x$ & $x$ & & \\
\hline [83] & $\mathrm{T} 2$ & & & $x$ & $x$ & $x$ & & & & & & & & & & & $x$ & $x$ & & \\
\hline [24] & $\begin{array}{l}\mathrm{T} 2, \mathrm{~S} 1 \\
\mathrm{~S} 2\end{array}$ & & & $x$ & $x$ & & $x$ & & & $x$ & $x$ & & & & & & $x$ & $x$ & $x$ & nUHPLC, adduct list \\
\hline [12] & $\mathrm{S} 1, \mathrm{~S} 2$ & & & $x$ & $x$ & & & & & $x$ & & & & & & & $x$ & $x$ & & \\
\hline
\end{tabular}




\begin{tabular}{|c|c|c|c|c|c|c|c|c|c|c|c|c|c|c|c|c|c|c|c|c|}
\hline Citation & Table & FA & FFA & GL & GP & LPL & PL & Ox & $\begin{array}{l}\text { Sulfo- } \\
\text { GP }\end{array}$ & SP & Hex & ST & $\mathrm{Ch}$ & SL & PK & PR & adduct & $\mathrm{m} / \mathrm{z}$ & CE & more \\
\hline [69] & T1 & & & $x$ & $x$ & & & & & $x$ & & & & & & & $x$ & $x$ & & \\
\hline [59] & $\mathrm{T} 2$ & & & $x$ & $x$ & & & & & & & $x$ & & & $x$ & & $x$ & $x$ & & Plant flavonoids included \\
\hline [2] & S1 & & & $x$ & $x$ & & & & & & & & & $x$ & $x$ & & $x$ & & & Plant flavonoids included \\
\hline [125] & $\begin{array}{l}\text { S1, } \\
\text { S2, S3 }\end{array}$ & & & $x$ & $x$ & & & & & & & & & $x$ & & $x$ & $x$ & $x$ & & $\begin{array}{l}\text { archaeal isoprene-based and ether-linked } \\
\text { lipid list (incl. fragment info) }\end{array}$ \\
\hline [18] & S3 & & & $x$ & $x$ & & & & & & & & & & & & $x$ & $x$ & & \\
\hline [50] & $\mathrm{T} 2, \mathrm{~T} 5$ & & & & $x$ & $x$ & & & & $x$ & & & & & & & $x$ & $x$ & & \\
\hline [47] & S1, S2 & & & & $x$ & & & & & $x$ & & & & & & & & $\mathrm{x}$ & & csv files (S2) \\
\hline [4] & T1 & & & & $x$ & & & & & & & & & & & & & $x$ & & \\
\hline$[61]$ & $\mathrm{T} 1$ & & & & $x$ & & & & & & & & & & & & $x$ & $x$ & & PC only \\
\hline [29] & S1 & & & & & & & $x$ & & & & & & & & & & $x$ & $x$ & Oxylipins \\
\hline$[55]$ & $\mathrm{T} 2, \mathrm{~S} 1$ & & & & & & & & & $x$ & & & $x$ & & & & $x$ & $x$ & & SM and cholesterol \\
\hline [40] & S2 & & & & & & & & & $x$ & & & & & & & $x$ & $x$ & & protonated species only \\
\hline [27] & S1 & & & & & & & & & & & $x$ & & & & & $x$ & $x$ & & Metabolomic study \\
\hline [44] & $\begin{array}{l}\text { A.144, } \\
\text { A.155 }\end{array}$ & & & & & & & & & & & $x$ & & & & & & & & SWATH \\
\hline [54] & S1 & & & & & & & & & & & & $\mathrm{x}$ & & & & & & & Math. model-assisted identification \\
\hline
\end{tabular}


Table 4. Databases used for lipid identification

\begin{tabular}{|c|c|c|c|}
\hline Database & Content & Focus & Used in Citation \\
\hline in-house & Compound lists & Lipids & {$[30],[32],[36],[51],[54],[68],[78]$} \\
\hline ChemSpider & Structures, Library & Comprehensive & [13], [127] \\
\hline Foodb & Library & Food constituents & [59] \\
\hline HMDB & Various & Metabolites & $\begin{array}{l}\text { [4], [12], [19], [32], [34], [39], [40], [59], [60], [63], } \\
{[127]}\end{array}$ \\
\hline KEGG & Pathway maps & Genes & {$[33],[34],[60],[79]$} \\
\hline LipidBlast & Spectra & Lipids & {$[6],[21],[25],[35],[76]$} \\
\hline LIPID MAPS & Library & Lipids & $\begin{array}{l}\text { [4], [6], [9], [12], [16], [19], [26], [34], [39], [40], [54], } \\
{[65],[69],[127]}\end{array}$ \\
\hline MassBank & Mass spectra & Comprehensive & {$[21]$} \\
\hline MetLin & MS/MS & Metabolites & [34], [39], [40], [63] \\
\hline NIST 11 & Library & Comprehensive & {$[60]$} \\
\hline Nist 14 & Library & Comprehensive & [25] \\
\hline PubChem & Library & Comprehensive & {$[13],[125]$} \\
\hline ChEBI* & Library & Metabolites & [125] \\
\hline Lipidbank & Compound lists & Lipids & [125] \\
\hline Reactome & Library & Pathways & {$[26]$} \\
\hline Home-made & Various & Various & {$[30],[32],[36],[54],[68]$} \\
\hline
\end{tabular}


Table 5. Coding languages, possible packages and specific softwares for analysis of lipids typically under the $\mathrm{m} / \mathrm{z}$ value of 1500 .

\begin{tabular}{|c|c|c|c|c|}
\hline Availability & Program & A/IQ/S/V & More & Used in Citation \\
\hline MathWorks & Matlab & $\mathbf{n} / \mathbf{y} / \mathbf{y} / \mathbf{y}$ & Coding language & [49] \\
\hline DoubleClick & Origin & $\mathbf{n} / \mathbf{n} / \mathbf{y} / \mathbf{y}$ & Coding language & {$[44],[75],[76]$} \\
\hline Open-source & Python & $\mathbf{n} / \mathbf{y} / \mathbf{y} / \mathbf{y}$ & Coding language & {$[25]$} \\
\hline \multirow[t]{4}{*}{ Open-source } & $\mathbf{R}$ & $\mathbf{n} / \mathbf{y} / \mathbf{y} / \mathbf{y}$ & $\begin{array}{l}\text { Coding language, } \\
\text { statistics }\end{array}$ & $\begin{array}{l}{[2],[3],[6],[7],[10],[18],[21],[32],[33],[36],} \\
{[37],[39],[40],[46],[47],[55],[65],[67],[68],} \\
{[74],[76],[78]}\end{array}$ \\
\hline & -Out of which CAMERA & $\mathbf{n} / \mathbf{y} / \mathbf{n} / \mathbf{n}$ & Isotope screening & [6], [21], [37], [55] \\
\hline & -Out of which MeV & $\mathbf{n} / \mathbf{y} / \mathbf{y} / \mathbf{y}$ & $\begin{array}{l}\text { DB search, } \\
\text { visualization }\end{array}$ & [3], [30] \\
\hline & -Out of which XCMS & $\mathbf{n} / \mathbf{y} / \mathbf{n} / \mathbf{n}$ & Feature detection & $\begin{array}{l}\text { [7], [10], [19], [32], [33], [36], [37], [39], [46], } \\
{[47],[55],[61],[65],[76],[78]}\end{array}$ \\
\hline IBM & SPSS & $\mathbf{n} / \mathbf{y} / \mathbf{y} / \mathbf{y}$ & $\begin{array}{l}\text { Coding language, } \\
\text { statistics }\end{array}$ & $\begin{array}{l}\text { [7], [26], [33], [42], [44], [50], [53], [54], [55], } \\
{[57],[60],[61],[64],[72],[75],[79]}\end{array}$ \\
\hline Agilent & Masshunter series & $\mathbf{y} / \mathbf{y} / \mathbf{n} / \mathbf{n}$ & Multiple tools & [7], [25], [34], [40], [54], [68] \\
\hline GraphPad & GraphPad Prism & $\mathbf{n} / \mathbf{n} / \mathbf{y} / \mathbf{y}$ & $\begin{array}{l}\text { Statistics, Data } \\
\text { visualization }\end{array}$ & {$[56],[57]$} \\
\hline KBSI & iLipid & $\mathbf{n} / \mathbf{y} / \mathbf{n} / \mathbf{n}$ & In-house & {$[14]$} \\
\hline Molecular Discovery & Lipostar & $\mathbf{n} / \mathbf{y} / \mathbf{y} / \mathbf{y}$ & $\begin{array}{l}\text { Identification } \\
\text { only }\end{array}$ & {$[65]$} \\
\hline N/A & LiPilot & $\mathbf{n} / \mathbf{y} / \mathbf{n} / \mathbf{n}$ & In-house & {$[24],[42]$} \\
\hline Open-source & CEU Mass Mediator & $\mathbf{n} / \mathbf{y} / \mathbf{n} / \mathbf{n}$ & $\begin{array}{l}\text { Adduct/s and RT, } \\
\text { website }\end{array}$ & [32], [63] \\
\hline Open-source & Cytoscape & $\mathbf{n} / \mathbf{n} / \mathbf{n} / \mathbf{y}$ & Visualization & [79] \\
\hline Open-source & MetaboAnalyst & $\mathbf{n} / \mathbf{y} / \mathbf{y} / \mathbf{y}$ & $\begin{array}{l}\text { Multipurpose, } \\
\text { website }\end{array}$ & $\begin{array}{l}\text { [10], [11], [17], [27], [32], [34], [37], [40], [46], } \\
{[48],[59],[60],[61],[63],[65],[77],[79]}\end{array}$ \\
\hline Open-source & Greazy & $\mathbf{n} / \mathbf{y} / \mathbf{y} / \mathbf{y}$ & $\begin{array}{l}\text { Chemometrics } \\
\text { included }\end{array}$ & [96] \\
\hline Open-source & KniMet & $\mathbf{n} / \mathbf{n} / \mathbf{y} / \mathbf{n}$ & Visualization & {$[68]$} \\
\hline Open-source & Lipid Data Analyzer & $\mathbf{n} / \mathbf{y} / \mathbf{y} / \mathbf{y}$ & $\begin{array}{l}\text { Standardization, } \\
\text { statistics }\end{array}$ & [9] \\
\hline Open-source & LipidFrag & $\mathbf{n} / \mathbf{y} / \mathbf{n} / \mathbf{n}$ & In silico & {$[21]$} \\
\hline Open-source & LipidMatch & $\mathbf{n} / \mathbf{y} / \mathbf{n} / \mathbf{n}$ & $\begin{array}{l}\text { In silico, } \\
\text { fragmentation } \\
\text { DBs }\end{array}$ & [3], [17], [63] \\
\hline
\end{tabular}

A/IQ/S/V: Acquisition/Identification \& Quantitation/Statistical analysis/data Visualization

*According to Waters' website, MassLynx is discontinued and replaced with Progenesis QI

DB: database, KBSI: Korea Basic Science Institute 


\begin{tabular}{|c|c|c|c|c|}
\hline Availability & Program & $\mathrm{A} / \mathrm{IQ} / \mathrm{S} / \mathrm{V}$ & More & Used in Citation \\
\hline Open-source & MassTRIX & $\mathbf{n} / \mathbf{y} / \mathbf{n} / \mathbf{y}$ & $\begin{array}{l}\text { KEGG/API } \\
\text { pathway analysis }\end{array}$ & {$[21]$} \\
\hline Open-source & MultiExperiment Viewer & $\mathbf{n} / \mathbf{n} / \mathbf{n} / \mathbf{y}$ & $\begin{array}{l}\text { Chemometric } \\
\text { visualization }\end{array}$ & {$[56]$} \\
\hline Open-source & MZmine & $\mathbf{n} / \mathbf{y} / \mathbf{n} / \mathbf{n}$ & MS DB search & {$[63],[123]$} \\
\hline Open-source & Skyline & $\mathbf{n} / \mathbf{y} / \mathbf{n} / \mathbf{n}$ & $\begin{array}{l}\text { MS/MS DB } \\
\text { search }\end{array}$ & [7], [34], [45], [69] \\
\hline Premier Biosoft & SimLipid & $\mathbf{n} / \mathbf{y} / \mathbf{n} / \mathbf{n}$ & $\begin{array}{l}\text { In silico structure } \\
\text { MS/MS }\end{array}$ & {$[10],[32],[61]$} \\
\hline Riken Prime & MS-DIAL & $\mathbf{n} / \mathbf{y} / \mathbf{n} / \mathbf{n}$ & SWATH & {$[35],[58],[73],[75-77]$} \\
\hline Sartorius & SIMCA & $\mathbf{n} / \mathbf{n} / \mathbf{y} / \mathbf{y}$ & $\begin{array}{l}\text { Statistics and } \\
\text { visualization }\end{array}$ & {$[5],[26],[33],[35],[36],[68],[79],[124]$} \\
\hline Sartorius & SIMCA-P & $\mathbf{n} / \mathbf{n} / \mathbf{y} / \mathbf{y}$ & Same as SIMCA & {$[7],[19],[30],[37],[52],[54],[56],[62]$} \\
\hline Sartorius & EZinfo & $\mathbf{n} / \mathbf{n} / \mathbf{n} / \mathbf{y}$ & Chemometrics & [4], [13], [34], [60], [69] \\
\hline SAS & JMP & $\mathbf{n} / \mathbf{n} / \mathbf{y} / \mathbf{y}$ & & {$[56]$} \\
\hline SCIEX & Analyst TF & $\mathbf{y} / \mathbf{n} / \mathbf{n} / \mathbf{n}$ & & {$[44],[33],[36]$} \\
\hline SCIEX & LipidView & $\mathbf{n} / \mathbf{y} / \mathbf{n} / \mathbf{n}$ & $\begin{array}{l}\text { Fragment DB } \\
\text { search }\end{array}$ & {$[16],[38],[43]$} \\
\hline SCIEX & MultiQuant & $\mathbf{n} / \mathbf{y} / \mathbf{n} / \mathbf{n}$ & Quantitation tool & [75], [76] \\
\hline SCIEX & PeakView & $\mathbf{n} / \mathbf{y} / \mathbf{n} / \mathbf{n}$ & $\begin{array}{l}\text { Peak comparison } \\
\& \text { analysis }\end{array}$ & [76] \\
\hline SCIEX & MarkerView & $\mathbf{n} / \mathbf{n} / \mathbf{y} / \mathbf{y}$ & & {$[38],[73],[76]$} \\
\hline Thermo & Xcalibur & $\mathbf{y} / \mathbf{n} / \mathbf{n} / \mathbf{n}$ & & $\begin{array}{l}{[2],[3],[5],[18],[26],[45],[46],[50],[58],[60],} \\
{[62],[65],[124]}\end{array}$ \\
\hline Thermo & Compound Discoverer & $\mathbf{n} / \mathbf{y} / \mathbf{y} / \mathbf{y}$ & Pathway analysis & [46] \\
\hline Thermo & LipidSearch & $\mathbf{n} / \mathbf{y} / \mathbf{n} / \mathbf{n}$ & \begin{tabular}{|l|} 
Relative \\
quantification
\end{tabular} & $\begin{array}{l}\text { [22], [26], [30], [35], [46], [50], [57], [62], [65], } \\
{[79],[124]}\end{array}$ \\
\hline Thermo & SIEVE & $\mathbf{n} / \mathbf{n} / \mathbf{y} / \mathbf{y}$ & $\begin{array}{l}\text { Biomarkers, } \\
\text { comparative } \\
\text { analysis }\end{array}$ & [19] \\
\hline Thermo & SIEVE & $\mathbf{n} / \mathbf{n} / \mathbf{y} / \mathbf{n}$ & $\begin{array}{l}\text { Biomarker } \\
\text { discovery }\end{array}$ & [19] \\
\hline Thermo & Chromeleon & $\mathbf{y} / \mathbf{n} / \mathbf{n} / \mathbf{n}$ & & {$[65]$} \\
\hline Waters & MassLynx MS & $\mathbf{y} / \mathbf{y} / \mathbf{n} / \mathbf{n}$ & & [7], [13-15], [28], [34], [60], [61], [69] \\
\hline Waters & MarkerLynx* & $\mathbf{n} / \mathbf{n} / \mathbf{n} / \mathbf{y}$ & Chemometrics & [4], [6], [16], [34], [43], [67] \\
\hline $\begin{array}{l}\text { Waters, Nonlinear } \\
\text { Dynamics }\end{array}$ & Progenesis QI & $\mathbf{n} / \mathbf{y} / \mathbf{n} / \mathbf{n}$ & $\begin{array}{l}\text { Small molecule } \\
\text { protein/lipid } \\
\text { analysis }\end{array}$ & [69] \\
\hline
\end{tabular}

A/IQ/S/V: Acquisition/Identification \& Quantitation/Statistical analysis/data Visualization

*According to Waters' website, MassLynx is discontinued and replaced with Progenesis QI

DB: database, KBSI: Korea Basic Science Institute 


\section{Figures:}

Figure 1. Simple triglyceride (TG) structure and shorthand notation. The stereospecific numbering (sn) of fatty acids on a glycerol-molecule are named sn1 (upper carbon of the glycerol body, if $\mathrm{k}>\mathrm{n}$ ), sn2 (middle carbon of the glycerol body) and sn3 (lower carbon of the glycerol body, if $\mathrm{k}>\mathrm{n}$ ) respectively, when the analyte can be stereoisomerically determined (i.e. on the stereo-molecular species level). If individual chain lengths (and their positions) cannot be determined, the number of the carbon atoms and double bonds are expressed as separate sums.

Figure 2. (A) Glycero- and glycerophospholipid structure variations according to sub-class and (B) regular and plasmalogen structures (O- for plasmanyl and $\mathrm{P}$ - for plasmenyl, older notation $e$ and $p$ ). The characteristic molecules of lipid classes are typically esterified to the sn3 position. Structures derived from ref. [93]

Figure 3. Typical sphingolipid structures. $R_{1}$ is an amine that usually has a fatty acid linked to it with an amide bond. $\mathrm{R}_{2}$ on the other hand is either a free hydroxyl group as in ceramides but occupied with a characteristic phosporylated molecule in SMs. Some structures also Structures derived from ref. [93]

Figure 4. '(a)' Nonpolar and '(b)' polar lipid subclass separation techniques. Reprinted from ref [85], DOI: 0958-1669/C 2016 S. Tumanov and JJ Kamphorst. Published by Elsevier Ltd, an open access article under the CC BY license. 


\section{Declaration of interests}

$\bigotimes$ The authors declare that they have no known competing financial interests or personal relationships that could have appeared to influence the work reported in this paper.

$\square$ The authors declare the following financial interests/personal relationships which may be considered as potential competing interests: 